\title{
A review of the discovery reach of directional Dark Matter detection
}

F. Mayet, ${ }^{1}$ A. M. Green,${ }^{2}$ J. B. R. Battat,${ }^{3}$ J. Billard ${ }^{4}$ N. Bozorgnia, ${ }^{5}$ G. B. Gelmini,${ }^{6}$ P. Gondolo, ${ }^{7}$ B. J. Kavanagh, ${ }^{8} 9$ S. K. Lee, ${ }^{10,11}$ D. Loomba, ${ }^{12}$ J. Monroe, ${ }^{13}$ B. Morgan, ${ }^{14}$ C. A. J. O'Hare, ${ }^{2}$ A. H. G. Peter, ${ }^{15,16}$ N. S. Phan, ${ }^{12}$ and S. E. Vahsen ${ }^{17}$

${ }^{1}$ Laboratoire de Physique Subatomique et de Cosmologie, Université Grenoble Alpes, CNRS/IN2P3, Grenoble, France

${ }^{2}$ School of Physics and Astronomy, University of Nottingham, University Park, Nottingham, NG7 2RD, UK

${ }^{3}$ Physics Department, Wellesley College, 106 Central Street, Wellesley, MA 02481, USA

${ }^{4}$ IPNL, Université de Lyon, Université Lyon 1, CNRS/IN2P3, 4 rue E. Fermi 69622 Villeurbanne cedex, France

${ }^{5}$ GRAPPA Institute, University of Amsterdam, Science Park 904, 1098 XH Amsterdam, Netherlands

${ }^{6}$ Department of Physics and Astronomy, University of California,

Los Angeles 475 Portola Plaza, Los Angeles, CA 90095, USA

${ }^{7}$ Dept. of Physics and Astronomy, University of Utah, 115 South 1400 East \#201, Salt Lake City, Utah 84112-0830

${ }^{8}$ Institut de Physique Théorique, Université Paris-Saclay, CNRS, CEA, F-91191 Gif-sur-Yvette, France

${ }^{9}$ Sorbonne Universités, UPMC Univ Paris 06, UMR 7589, LPTHE, F-75005, Paris, France

${ }^{10}$ Princeton Center for Theoretical Science, Princeton University, Princeton, NJ 08544, USA

${ }^{11}$ Broad Institute, Cambridge, MA 02142, USA

${ }^{12}$ Physics $\&$ Astronomy Dept., University of New Mexico, 1919 Lomas Blvd NE, Albuquerque, NM 87131 USA

${ }^{13}$ Department of Physics, Royal Holloway, University of London, Egham Hill, Surrey, TW20 OEX, UK

${ }^{14}$ Department of Physics, University of Warwick, Gibbet Hill Road, Coventry, CV4 7AL, UK

${ }^{15}$ CCAPP and Department of Physics, The Ohio State University, 191 W. Woodruff Ave., Columbus, OH 43210, USA

${ }^{16}$ Department of Astronomy, The Ohio State University, 140W. $18^{\text {th }}$ Ave., Columbus, OH 43210, USA

${ }^{17}$ Department of Physics and Astronomy, University of Hawaii, 2505 Correa Road, Honolulu, HI 96822, USA

(Dated: March 7, 2016)

Cosmological observations indicate that most of the matter in the Universe is Dark Matter. Dark Matter in the form of Weakly Interacting Massive Particles (WIMPs) can be detected directly, via its elastic scattering off target nuclei. Most current direct detection experiments only measure the energy of the recoiling nuclei. However, directional detection experiments are sensitive to the direction of the nuclear recoil as well. Due to the Sun's motion with respect to the Galactic rest frame, the directional recoil rate has a dipole feature, peaking around the direction of the Solar motion. This provides a powerful tool for demonstrating the Galactic origin of nuclear recoils and hence unambiguously detecting Dark Matter. Furthermore, the directional recoil distribution depends on the WIMP mass, scattering cross section and local velocity distribution. Therefore, with a large number of recoil events it will be possible to study the physics of Dark Matter in terms of particle and astrophysical properties. We review the potential of directional detectors for detecting and characterizing WIMPs.

PACS numbers: 95.35.+d, 14.80.-j

(C) 2016. This manuscript version is made available under the Elsevier user license http://www.elsevier.com/open-access/userlicense/1.0/ 


\section{Introduction}

II. Experimental framework

A. Introduction

B. Track reconstruction

C. Energy measurement

D. Residual background contamination

E. Current experimental landscape

1. DRIFT

2. MIMAC

3. NEWAGE

4. DMTPC

5. R\&D toward improved $3 \mathrm{~d}$ readout

F. Conclusion

III. Theoretical framework
A. Introduction
B. Physics of the Galactic halo
C. Event rate and cross section
D. Inputs from nuclear physics
E. Parameters from particle physics models
F. Non-relativistic effective theory

5

6

7

7

IV. Directional features

A. Introduction $\quad 13$

B. Dipole feature $r$

C. Ring-like features $\quad 15$

D. Aberration features $\quad 16$

V. Setting limits on WIMP-nucleon scattering $r$

A. Introduction $\quad 17$

B. Maximum Patch Method 18

$\begin{array}{lr}\text { C. Likelihood Test } & 19\end{array}$

$\begin{array}{ll}\text { D. } \chi^{2} \text { Test } & 20\end{array}$

$\begin{array}{lr}\text { VI. Directional discovery } & 20\end{array}$

$\begin{array}{ll}\text { A. Introduction } & 20\end{array}$

B. Detecting anisotropy 21

C. Measuring the mean recoil direction $\quad 21$

D. Experimental considerations 22

E. Using directionality to overcome the neutrino floor 24

$\begin{array}{ll}\text { VII. Observing secondary features } & 27\end{array}$

A. Introduction $\quad 27$

B. Observing ring-like features $\quad 27$

$\begin{array}{ll}\text { C. Observing aberration features } & 28\end{array}$

VIII. Dark matter identification $\quad 31$

A. Introduction $\quad 31$

B. Comparing directional and direction-insensitive detection $\quad 32$

C. Constraining halo and WIMP properties 32

1. Constraining Dark Matter halo properties

2. Constraining WIMP properties 33

IX. Probing the interaction of Dark Matter $\quad 35$

A. Introduction $\quad 35$

B. Using directionality to constrain inelastic Dark Matter 35 
X. Searching for light WIMPs 37

A. Introduction 37

B. Theoretical studies 37

C. Experimental prospects 38

D. Conclusions 39

XI. Probing halo substructure 39

A. Introduction 39

B. Streams 40

1. Stream detectability 41

2. Constraining stream parameters 43

C. Dark disk 43

1. Constraining dark-disk parameters 43

XII. Decomposing the velocity distribution 45

A. Introduction 45

B. Moment decomposition 45

C. Fourier-Bessel decomposition 46

D. Discretized decomposition 47

XIII. Conclusion 48

XIV. Acknowledgements 49

A. Transformation from the detector frame to the Galactic frame 49

1. Galactic rotation 50

2. Solar motion 51

3. Earth's revolution 51

4. Earth's rotation $\quad 52$

5. Total Velocity 52

References $\quad 53$ 


\section{INTRODUCTION}

A multitude of astrophysical and cosmological observations, involving different physical processes at diverse scales, provide evidence for the existence of non-baryonic Dark Matter in the Universe and, in particular, in the halo of our Galaxy. These observations also provide hints to the properties of Dark Matter. Dark Matter makes up $27 \%$ of the total mass-energy density of the Universe [1]. This precise measurement constrains the Dark Matter production mechanism in the early Universe and its interaction cross section. The fact that halos of Dark Matter surround galaxies and extend an order of magnitude in distance beyond the visible component of the galaxies implies that Dark Matter experiences at most weak dissipative interactions [2,3]. Since estimates of the Dark Matter density at the epoch of the cosmic microwave background are consistent with present-day observations, Dark Matter must have a lifetime larger than the Hubble time. Together these observations suggest that Dark Matter consists of at least one quasi-stable particle, that is not part of the Standard Model of particle physics. One of the best motivated particle Dark Matter candidates is a stable, massive, neutral particle generically referred to as a WIMP (Weakly Interacting Massive Particle) [4-6].

The search for Dark Matter is at the interface between astrophysics and particle physics. In astrophysics its gravitational influence appears at all scales (from galaxies to large-scale structures), while extensions of the Standard Model of particle physics suggest the existence of a particle with the characteristics of a WIMP. Several WIMP detection techniques have been proposed and are being pursued. Searches for laboratory-created WIMPs are ongoing at the Large Hadron Collider [7], as are attempts to detect Standard Model particles produced by WIMP annihilation in our own and extragalactic Dark Matter halos [8]. Direct detection experiments aim to detect Galactic halo WIMPs streaming through terrestrial detectors, via their elastic scattering off target nuclei.

Direct detection experiments are now approaching ton-scale in mass [9]. However, the sensitivity of these experiments to WIMPs is subject to both reducible (environmental) and irreducible (neutrino) backgrounds [10], which may mimic WIMPs. To unambiguously claim a WIMP detection, it will thus be necessary to demonstrate that the observed events are not due to backgrounds. Electron-induced recoils may be rejected efficiently, see Ref. [9] for a review. However discriminating neutron and WIMP-induced nuclear recoils remains a challenging issue. One potential tool to disentangle the WIMP signal from the neutron background is the annual modulation signal [11-13], which is due to the Earth's orbital motion about the Sun. The WIMP speed distribution in the lab-frame, and hence the energy spectrum of WIMP-induced recoils, varies annually. However, the modulation amplitude is small [14] and it is possible that backgrounds also modulate annually.

The interest in directional detection is based on the unique directional signature of Galactic Dark Matter, which enables an unambiguous separation of signal and backgrounds. Since the Sun moves, with respect to the Galactic Dark Matter halo, in a nearly circular orbit with velocity $v_{\mathrm{c}} \approx 220 \mathrm{~km} \mathrm{~s}^{-1}[15,16]$, the WIMP-induced nuclear recoil spectrum is expected to be sharply peaked in the direction of motion of the Sun. This is generally referred to as the dipole feature (see Sec. IV B). The strength of directional detection lies in the fact that the is no reason for the neutron-induced nuclear recoil spectrum to have a dipole anisotropy. Directional detection requires the measurement of both the energy and the direction of the recoiling nucleus and is a next generation strategy for direct detection.

The idea of directional detection was first proposed by D. N. Spergel in 1988 [17]. Since then the interest in directional detection has grown, due to technological advances that make the construction of a large directional detector possible in the near future. In this review we focus on the discovery reach of directional detection and address the following question: What unique contributions can directional detection make in the search for Dark Matter? This is obviously a major issue, given the current worldwide effort aimed at building a large directional detector and the timescales required to do so.

In Sec. II we briefly introduce the key experimental issues that must be addressed in order to perform a directional Dark Matter search. As the focus of this review is on theoretical ideas for directional searches, we only include the technical details required to place the remaining sections in proper context. We refer the reader to Refs. [18-20] for a complete overview of the instrumental side of the subject. Section III presents the relevant theoretical framework for both Galactic and particle physics. In Sec. IV we present the main directional feature, the so-called dipole, due to the Sun's motion through the Galactic halo and also two secondary directional features: the ring-like and aberration features. Section V studies directional detection as a tool to set limits on the WIMP-nucleon scattering rate in the absence of a significant signal. The main power of directional detection, however, is in the discovery of Dark Matter. Section VI demonstrates that directional detectors can confirm the Galactic origin of recoils at high significance, and hence make a definitive discovery of Dark Matter. In particular we discuss how directionality can overcome the 
floor in the sensitivity of conventional (non-directional) direct detection experiments due to neutrino backgrounds. In Sec. VII we study the prospects of observing the ring-like and aberration features. After the convincing discovery of WIMPs by at least one experiment, the next goal will be to constrain the properties of both the WIMP particle (mass and cross section) and the Galactic Dark Matter halo (three dimensional local WIMP velocity distribution and density). This topic is addressed in Sec.VIII. The capability of a directional detector to probe the underlying particle physics model is investigated in Sec. IX. Motivated by recent experimental and theoretical interest in light WIMPs, the potential of directional detectors to detect light WIMPs is studied in Sec. X. The use of directional detection to constrain the astrophysical properties of the Dark Matter velocity distribution, including substructures, is an exciting possibility that is presented in Sec. XI. Finally, in Sec. XII we present a technique to decompose the WIMP velocity distribution, enabling a model-independent analysis of directional data.

\section{EXPERIMENTAL FRAMEWORK}

\section{A. Introduction}

In this section, we briefly adress the key experimental issues for directional WIMP detection. For a detailed review of the status of directional detectors, we refer the reader to Refs. [18-20].

Directional detection aims to measure both the energy and the direction of the recoiling nuclei. Hence, it constitutes an extension of direction-insensitive direct detection, for which only the energy is measured. Several directional detection strategies have been proposed: low pressure gaseous Time Projection Chambers (TPCs) [21-29], nuclear emulsions [30, 31], columnar recombination in high-pressure gaseous Xenon TPCs [32-34], solid-state detectors [3537], DNA-based detectors [38], graphene-based heterostructures [39] and polarized detectors [40]. Not all techniques are equally mature. Some are at the level of concepts or of advanced R\&D, others have led to the construction of detectors that are already operating underground [27, 29, 41]. As for direction-insensitive detection, the exposure, and hence the mass of the detector, is a key issue to reach low cross sections. Directional detectors thus face the scale-up challenge, in addition to that of directional sensitivity. The cubic metre scale has been operated by the DRIFT detector [29] and DMTPC, but significantly larger directional detectors must be constructed to reach leading sensitivity.

In the following, we discuss the main experimental issues that directional detection faces: the track reconstruction (Sec. II B), the energy measurement (Sec. II C) and the residual background contamination (Sec. II D). To illustrate the current state of the art, we give examples of difficulties and achievements, mainly focusing on directional TPCs that currently constitute the most mature directional technology.

\section{B. Track reconstruction}

The recoil track may be reconstructed either in $1 \mathrm{~d}, 2 \mathrm{~d}$, or $3 \mathrm{~d}$, depending on the choice of the readout technology. Let $\hat{\mathbf{r}}$ be the recoil direction given by

$$
\hat{\mathbf{r}}=\sin \vartheta \cos \varphi \hat{\mathbf{x}}+\sin \vartheta \sin \varphi \hat{\mathbf{y}}+\cos \vartheta \hat{\mathbf{z}},
$$

where the Earth-fixed $\hat{\mathbf{x}}, \hat{\mathbf{y}}$, and $\hat{\mathbf{z}}$ axes point toward the North, the West and the Zenith directions respectively. For a $1 \mathrm{~d}$ track reconstruction, the track is projected along the $z$-axis and hence the $\vartheta$ angle is measured. For a $2 \mathrm{~d}$ track reconstruction, $\varphi$ is measured, e.g. by projecting the track onto the $(x, y)$ plane of the detector. A $3 \mathrm{~d}$ track reconstruction requires that both the $\varphi$ and $\vartheta$ angles are reconstructed. Note, that an equatorial mount is considered for some detectors [42].

Directional TPCs are operated at low pressure so that the low energy recoil track is long enough to be detected on the readout plane. $3 \mathrm{~d}$ track reconstruction can be achieved with a time-sampling of the $2 \mathrm{~d}$-projection of the ionizationinduced electron cloud on a pixelized readout plane. Conversely, low pressure limits the achievable exposure, but small scale $3 \mathrm{~d}$-detectors, $\sim 0.1 \mathrm{~kg}$, are already in operation [26]. 1d-directional detection could be achieved with liquid or even solid-state detectors [36, 37], hence a priori enhancing the exposure. However, no 1d liquid/solid detector has been operated and there is yet no experimental evidence that columnar recombination is measurable for keV nuclear recoils.

The choice of the readout strategy has consequences on the Dark Matter reach, as studied in Ref. [43] and presented in Sec. VI. 
It is also desirable to retrieve the sense of the track of the recoiling nucleus $(+\hat{\mathbf{r}}$ vs. $-\hat{\mathbf{r}})$. Sense recognition may be achieved thanks to an asymmetry between $+\hat{\mathbf{r}}$ and $-\hat{\mathbf{r}}$ tracks due to:

i) the angular dispersion of recoiling tracks. It means that the beginning of the track is more rectilinear than its end [44]. Using this feature is challenging due to the angular dispersion, which increases with decreasing energy.

ii) charge collection asymmetry. Indeed, $\mathrm{d} E / \mathrm{d} x$ decreases with energy at low recoil energies. Consequently, more primary electrons are generated at the beginning of the track. The effect is often referred to as the head-tail effect. Note that to measure the head/tail charge asymmetry, a readout that gives energy versus position on the track is required.

Although sense recognition has been demonstrated experimentally [45-47], it remains a challenging issue for directional detection. This is because the intrinsic asymmetry in $\mathrm{d} E / \mathrm{d} x$ has significant fluctuations, and with a non-negligible angular dispersion of the recoil nucleus and diffusion of its track in the detector medium the ability to determine the sense is compromised. As such, directional data are expected to be only partialy sense-recognized, with an efficiency strongly depending on the energy and the drift distance [44]. With no sense recognition, the expected WIMP distribution is a double dipole (see Sec. IV) that is closer to the expected isotropic background event distribution and hence harder to discriminate [48].

The main goal of the track reconstruction is the estimation of the initial recoil direction, as this is directly linked to the kinematics of the WIMP scattering. Once the recoiling nucleus encounters its first elastic scattering off another nucleus from the sensitive medium, the knowledge of the initial recoil direction starts to get lost. Eventually, the successive scatters give rise to a non-negligible angular dispersion, usually referred to as the straggling effect, which constitutes an intrinsic limitation to directional detectors. For instance, in the case of $\mathrm{CF}_{4}$ gas, $\mathrm{F}$ recoils can suffer from an intrinsic angular dispersion from straggling effect of about $25 \mathrm{deg}$ (rms) at $10 \mathrm{keV}$ [49].

For low pressure gaseous TPCs, the main feature is transverse and longitudinal diffusion of the charge cloud during the drift [50] from the scattering vertex to the readout plane. For small drift distances, it constitutes an intrinsic limitation to the angular resolution. For long drift distances, the charge cloud is being so diluted that the reconstruction of the track properties is no longer feasible. For a given instrumental configuration (gain, pressure), this effect sets a limit on the drift distance, hence to the mass of the detector and consequently to the achievable exposure for a single module. The choice of the target medium is thus critical. $\mathrm{CS}_{2}$ offers the possibility to drift negative ions, which enables very long drift distances with minimal diffusion and hence large volumes [51-57]. Directional electron-drift TPCs are using $\mathrm{CF}_{4}$, for the spin content of ${ }^{19} \mathrm{~F}$ that enables a sensitivity to spin dependent interaction [58], and for the properties of this gas: electron drift velocity, diffusion and its scintillation output, which can be used for optical readout $[22,59] . \mathrm{SF}_{6}$ is also being studied, as it may combine the advantages of $\mathrm{CS}_{2}$ and $\mathrm{CF}_{4}$, i.e. a target with a non-vanishing spin in an electronegative gas [60]. Note that the angular resolution of directional detectors must be evaluated through detector commissioning, using an ion beam or neutron field [61], and accounted for in dedicated data analyses [44].

The reconstruction of the localization of the vertex of the WIMP-nucleus scattering in the detector volume is also important in order to discriminate WIMP-induced recoils from surface events, which mostly come from the radioactivity of the surrounding detector material [41, 44, 62-65].

The impact of track reconstruction (angular resolution, sense recognition) on the reach of directional detection is evaluated in Secs. V and VI.

\section{Energy measurement}

The issue of energy measurement is not specific to direction-sensitive direct detection. In particular, the quenching of the deposited energy must be accounted for to convert a measured energy, usually expressed in electron-equivalent energy (e.g. $\mathrm{keV}_{\mathrm{ee}}$ ), into a recoil energy $E_{r}$, expressed in recoil energy $\left(\mathrm{keV}_{\mathrm{r}}\right)$, see e.g. Ref. [66]. As for directioninsensitive direct detection, the energy threshold plays a key role for directional detection. It is even more challenging for directional detection, as in this case the energy threshold is the lowest energy at which both the initial recoil direction and the energy can be measured. In a low-pressure gaseous detector, a keV nuclear recoil has a short track length and encounters a rather large diffusion during the drift to the readout plane. Hence, the directional energy threshold depends on the choice of the target, the gas mixture, the signal strength (gas gain) and the read-out strategy.

In the following, we present the impact of the energy threshold on the capability of a directional detector to exclude (Sec. V) or discover (Sec. VI) Dark Matter. 


\section{Residual background contamination}

As for a direction-insensitive detector, the discrimination of background electron recoils from nuclear recoils is a key issue for a directional detector [23, 29, 67, 68]. However, as neutrons lead to a nuclear recoil, just as WIMPs do, they are usually considered as an irreducible background for the direct detection of Dark Matter. The strength of directional detection lies in the fact that the WIMP signal exhibits a unique feature, as the WIMP-induced nuclear recoil spectrum is expected to be peaked in the direction of motion of the Sun, see Sec. IV. Hence, one expects a WIMP dipole feature in Galactic coordinates, while there is no reason for the neutron-induced nuclear recoil spectrum to present the same feature.

Moreover, the neutron background is expected to be isotropically distributed. Neutrons in underground laboratories originate either from spontaneous fissions and $(\alpha, n)$ reactions, or from cosmic muon interactions. Whereas the former are associated with an isotropic neutron distribution in the laboratory frame, the latter need a careful evaluation. D. Mei et al. have shown that the muon-induced neutron angular distribution is mainly flat with respect to $\cos \theta$, $\theta$ being the zenith angle, with a small forward excess due to the spallation process [69]. As noted in Ref. [70], a small anisotropy in the laboratory rest-frame will be erased when considering the Galactic rest-frame, due to the Earth rotation and orbit. Moreover, any residual anistropy would be further smeared as directional detection is sensitive to the induced recoils, and not the neutrons themselves. Under the isotropy hypothesis, a likelihood analysis of directional data is possible [71]. In fact, the neutron background only has to be known and a full Monte Carlo evaluation of the neutron angular distribution in underground laboratories would be a valuable contribution to the field, to assess the level of isotropy of the neutron background in Galactic coordinates. Note that the situation is similar for other backgrounds ( $\gamma$, electrons), but they can be discriminated from nuclear recoils [23, 29, 67, 68], or included in a likelihood analysis. Eventually, we emphasize that the isotropy of the neutron background is not a sine qua non condition for directional detection. Only the WIMP dipole feature is mandatory and it is due to the Sun's motion with respect to the Dark Matter halo.

In the following, we show that the directional detection reach is only mildly degraded by a small background contamination (Sec. VI), thanks to the intrinsic difference between the neutron- and WIMP-induced angular recoil spectra. To quantifiy the level of residual background in the data, we define the WIMP fraction as

$$
\lambda=S /(B+S)
$$

where $S$ and $B$ are the number of signal and background events respectively.

Finally, the neutrino background is considered to be the ultimate background for the direct detection of Dark Matter [10, 72-74]. In Sec. VIE, we present how to overcome the neutrino floor by using directionality.

\section{E. Current experimental landscape}

For completeness, we conclude this section with an overview of the directional TPCs that are currently being operated underground, or planned to start running underground in the near future, in terms of detection strategies, achievements and future developments, see Tab. I. We also present a short overview of current R\&D efforts toward spatial-resolution and/or higher signal-to-noise ratio.

\section{DRIFT}

The DRIFT collaboration has developped the first low-pressure gas TPC for directional Dark Matter detection [75]. The key feature of the DRIFT detector is the fact that negative ions $\left(\mathrm{CS}_{2}^{-}\right)$are used to transport the ionisation signal to the readout plane composed of Multi-Wire Proportional Counters (MWPC) [57]. It opens the possibility to develop TPCs with very long drift distances with minimal diffusion and hence large volumes [53-57]. The gas mixture currently used is $73 \% \mathrm{CS}_{2}+25 \% \mathrm{CF}_{4}+2 \% \mathrm{O}_{2}$ at a pressure of 55 mbar. $\mathrm{CF}_{4}$ is used to enable the sensitivity to SD interaction, while the small $\mathrm{O}_{2}$ component [76] enables the measurement of the absolute position of the interaction vertex in the drift direction [29]. The achieved threshold is $20 \mathrm{keV}$ [24] and a factor of 2 higher in the analysis presented in [29], due to the use of minority carriers. The DRIFT collaboration has demonstrated, through several R\&D stages and underground runs at the Boulby mine, stable operation of a $\mathrm{m}^{3}$ detector and has recently published the first background-free operation of a directional dark matter detector, thanks to a full-volume fiducialisation [29]. The collaboration aims to develop a directional detector at the ton scale [77]. 


\begin{tabular}{|c||c|c|c|c|c|c|c|}
\hline Exp. & $\mathrm{V}(\mathrm{L})$ & Gas & $\mathrm{P}(\mathrm{mbar})$ & Drift & Threshold $(\mathrm{keV})$ & Location & Ref. \\
\hline \hline DRIFT & 800 & $73 \% \mathrm{CS}_{2}+25 \% \mathrm{CF}_{4}+2 \% \mathrm{O}_{2}$ & 55 & ion, $50 \mathrm{~cm}$ & $20[24]$ & Boulby & {$[29]$} \\
\hline MIMAC & 5.8 & $70 \% \mathrm{CF}_{4}+28 \% \mathrm{CHF}_{3}+2 \% \mathrm{C}_{4} \mathrm{H}_{10}$ & 50 & $e^{-}, 20 \mathrm{~cm}$ & 2 & Modane & {$[86]$} \\
\hline NEWAGE & 37 & $\mathrm{CF}_{4}$ & 100 & $e^{-}, 41 \mathrm{~cm}$ & 50 & Kamioka & {$[87]$} \\
\hline DMTPC & 1000 & $\mathrm{CF}_{4}$ & 40 & $e^{-}, 27 \mathrm{~cm}$ & 20 & SNOLAB & {$[91,93]$} \\
\hline
\end{tabular}

TABLE I: Main properties of the directional experiments that are currently being operated underground or start running underground in the near future.

\section{2. $M I M A C$}

The MIMAC collaboration is developing an electron-drift TPC [78, 79] that aims at 3d track reconstruction [44]. The measurement of the third dimension, along the electric field, is achieved thanks to sampling of the primary electron cloud that is projected onto the grid of a bulk micromegas [80,81] with a read-out at a frequency of $50 \mathrm{MHz}$ by a self-triggered electronics $[82,83]$. The gas mixture chosen is $70 \% \mathrm{CF}_{4}+28 \% \mathrm{CHF}_{3}+2 \% \mathrm{C}_{4} \mathrm{H}_{10}$, which enables sensitivity to SD interaction with a sufficient gain and reduced drift velocity $[84,85]$. A prototype is currently being operated underground, at the LSM laboratory (Modane), at a pressure of 50 mbar with an active volume of $5.8 \mathrm{~L}$. The achieved threshold is $2 \mathrm{keV}$ [86]. The short-term goal is the building of a $1 \mathrm{~m}^{3}$ detector that will be the demonstrator of a large TPC devoted to directional detection $\left(50 \mathrm{~m}^{3}\right)$. The current $5.8 \mathrm{~L}$ detector is meant to be the elementary cell of the matrix structure. Larger micromegas detectors $\left(20 \times 20 \mathrm{~cm}^{2}\right)$ are also being studied [86].

\section{NEWAGE}

The NEWAGE collaboration is developing an electron-drift TPC that has been operated underground at Kamioka, at various $\mathrm{R} \& \mathrm{D}$ stages. The TPC is characterized by a $41 \mathrm{~cm}$ drift distance, readout plane made of $\mu$-PIC (micro-pixel chamber) and a gas electron multiplier (GEM). The gas is pure $\mathrm{CF}_{4}$ at $100 \mathrm{mbar}$. The achieved threshold is $50 \mathrm{keV}$ [87]. The performance of the detector has been studied in Ref. [87]. A ${ }^{252} \mathrm{Cf}$ neutron source has been used to evaluate the angular resolution, which is $40^{\circ}$ at $50 \mathrm{keV}$, and the detection efficiency, which is of the order of $40 \%$ at $50 \mathrm{keV}$ and reaches $90 \%$ at $350 \mathrm{keV}$. The electron rejection power, which is $2.5 \times 10^{-5}$ between 50 and $100 \mathrm{keV}$, has been evaluated with a ${ }^{137} \mathrm{Cs}$ source. In 2007, the NEWAGE collaboration published the first direction-sensitive dark matter limit [27] obtained from a dedicated run in an underground laboratory at Kamioka mine. In 2015, they improved their result by a factor of ten with a 31.6 day run corresponding to an exposure of $0.327 \mathrm{~kg}$ - days. It leads to the first experimental recoil map, presenting the observed background events in galactic coordinates, which is the first step toward directional measurement of WIMP and background events.

\section{4. $D M T P C$}

The DMTPC collaboration is developing an electron-drift TPC filled with $\mathrm{CF}_{4}$ at $40-100$ mbar. The readout uses CCD cameras together with low noise amplifiers to measure both optical $[22,88]$ and electronic signals from particles in the detector $[28,68]$. The current amplifier is sensitive to the rise time of the electron peak, enabling the possibility to estimate the position of the track in the direction of the field [23]. DMTPC demonstrated the first measurement of the vector track direction using the head-tail effect [89], and has achieved $75 \%$ efficiency for vector direction reconstruction for tracks with minimum aspect ratio of 3:1 (length:width) [90, 91]. A 10 L prototype set a spin-dependent WIMP scattering limit and demonstrated $40^{\circ}$ recoil angle resolution at $80 \mathrm{keVr}$ threshold [22], and was operated underground at WIPP for assay of intrinsic detector backgrounds [92]. A $1 \mathrm{~m}^{3}$ detector has been built, is currently commissioning at the surface with 30 mbar CF4 pressure for $20 \mathrm{keV}_{\mathrm{r}}$ threshold [91, 93]; underground operation is planned at SNOLAB. It is intended to be the elementary cell of a larger detector.

\section{RED toward improved $3 d$ readout}

In addition to the above experiments, several groups have constructed prototype detectors to demonstrate candidate technologies for achieving 3d readout of directional TPCs with improved spatial-resolution and/or higher signal-to- 
noise ratio. Examples are prototypes with thick GEMS at the University of New Mexico [59], the $D^{3}$ prototypes with ATLAS pixel chip readout at the University of Hawaii [51, 94], the NITEC prototypes with CERN Timepix chip readout at INFN, and work on high-density strip readout at Wellesley College. The overall status of readout R\&D for directional dark matter detectors is the subject of a separate, forthcoming review article [95].

\section{F. Conclusion}

Finally, we note that the most suitable readout for a large dark matter detector may not be the one with the highest performances as that will be the most costly choice per target volume. Hence one needs to make the best tradeoff between cost and sensitivity, to maximize sensitivity per unit cost.

Eventually, it should be noted that there is a worldwide effort toward the development of large TPC for the directional detection of Dark Matter that aimed at combining the main advantages and achievements of the detectors above presented. The project is still in discussion and could be done either in parallel with current instrumental developments or could superseed them within the framework of a single large TPC [96].

\section{THEORETICAL FRAMEWORK}

\section{A. Introduction}

The directional detection strategy is based on the fact that the Sun is orbiting the Galactic centre and is currently travelling towards the constellation Cygnus. If the WIMP distribution is predominantly smooth, the Sun's speed and the velocity dispersion of the WIMPs are expected to be similar in magnitude, and therefore the WIMP flux is concentrated around the direction of Solar motion. As direction-insensitive direct detection, directional detection is also based on the scattering of a Dark Matter particle on a target nucleus of the detector. In particular, it determines the kinematics of the nuclear recoil and the event rate.

This section is devoted to the basic concepts, from both astrophysics and particle physics, needed to evaluate the double-differential spectrum, i.e. the number of events per unit recoil energy in a given direction. This contains the information about Dark Matter that directional detection aims to extract: the WIMP velocity distribution (Sec. III B), mass and cross section (Sec. III C). Sections III D and III E introduce the parameters from nuclear and particle physics that enter the evaluation of the event rate. We first focus on a WIMP with a standard SUSY-type elastic interaction, then Sec. III F presents a theoretical framework that opens the possibility of distinguishing different Dark Matternucleon interactions.

\section{B. Physics of the Galactic halo}

Historically, direct detection signal calculations and data analyses have used the Standard Halo Model (SHM) to model the WIMP velocity distribution. This simple model assumes that the Milky Way (MW) halo is an isotropic, isothermal sphere with density profile $\rho \propto r^{-2}$. In this case, the normalised velocity distribution in the rest frame of the Galaxy takes the form of a Maxwellian distribution

$$
f_{\text {gal }}(\mathbf{v})= \begin{cases}\frac{N}{\left(2 \pi \sigma_{v}\right)^{3 / 2}} \exp \left(-\frac{|\mathbf{v}|^{2}}{2 \sigma_{v}^{2}}\right) & \text { if }|\mathbf{v}|<v_{\text {esc }} \\ 0 & \text { if }|\mathbf{v}|>v_{\text {esc }}\end{cases}
$$

where $N$ is a normalisation constant. In the SHM, the one-dimensional velocity dispersion, $\sigma_{v}$, is independent of the distance from the Galactic centre and is related to the circular speed, $v_{\mathrm{c}}$, via $\sigma_{v}=v_{\mathrm{c}} / \sqrt{2}$. Formally, the Maxwellian distribution extends to infinity; however, in the case of the SHM it is usually truncated by hand at the local escape speed, $v_{\text {esc }}$.

The canonical value of the local circular speed is $v_{\mathrm{c}}=220 \mathrm{~km} \mathrm{~s}^{-1}$, with a statistical error of order $10 \%[15,16,97]$. However the assumptions made when modelling the MW halo, for instance that the MW rotation curve is flat, lead to significant systematic uncertainties. Considering various models for the MW rotation curve leads to determinations of $v_{\mathrm{c}}$ which range from $(200 \pm 20) \mathrm{km} \mathrm{s}^{-1}$ to $(279 \pm 33) \mathrm{km} \mathrm{s}^{-1}$ [98]. Note that in general the relationship between the circular speed and the velocity dispersion depends on the density profile and the velocity anisotropy (see e.g. Ref. [99]). Assuming a smooth halo in equilibrium, the RAVE survey has recently found the escape speed to be $v_{\text {esc }}=537_{-43}^{+59} \mathrm{~km} \mathrm{~s}^{-1}[100]$. The local WIMP density, $\rho_{0}$, also enters the calculation of the recoil spectrum. The 
value traditionally used in WIMP direct detection calculations is $\rho_{0}=0.3 \mathrm{GeV} \mathrm{cm}{ }^{-3}$. Recent analyses, which use a range of dynamical data sets to constrain a mass model of the Milky Way, typically find a slightly higher value, $\rho_{0} \approx 0.4 \mathrm{GeV} \mathrm{cm}^{-3}[101,102]$, with a statistical error of order $10 \%$. These analyses assume a Navarro Frenk White density profile [103] for the Dark Matter halo. Considering a wider range of halo profiles leads to local densities in the range $\rho_{0}=(0.2-0.6) \mathrm{GeV} \mathrm{cm}^{-3}[104-107]$. Local estimates of the DM density, which do not require global mass-modelling of the Milky Way (e.g. Refs. [108, 109]), are generally in agreement with these values, though with larger errors.

To calculate the recoil spectrum, the velocity distribution needs to be translated into the lab frame. Formally, this should be done using Liouville's theorem [110, 111]. However the gravitational potential of the Sun and Earth only affect the very low speed WIMPs $\left(v \lesssim 40 \mathrm{kms}^{-1}\right)[110]$, and therefore a Galilean transformation $f(\mathbf{v}, t)=$ $f_{\text {gal }}\left(\mathbf{v}+\mathbf{v}_{\text {lab }}(t), t\right)$, where $\mathbf{v}_{\text {lab }}(t)$ is the lab's velocity with respect to the Galactic rest frame, is sufficient. The lab's velocity is the sum of the local circular speed, the Sun's motion with respect to the Local Standard of Rest, and the Earth's velocity relative the Sun. The full, correct calculation of the transformation between the reference frames is carried out in Refs. [112, 113], see also Appendix A. Note that, as pointed out in Ref. [113], the commonly used expressions from Ref. [14] contain an error.

The SHM may not to be a good approximation to the real MW halo, as the assumptions behind it (that the MW halo is an isotropic, isothermal sphere in dynamical equilibrium) are not valid. In cold Dark Matter cosmologies, structures form hierarchically, leading to DM halos which are triaxial, anisotropic and contain phase-space substructure (see e.g. Ref. [114]). The velocity distributions of high-resolution Dark Matter-only simulations of MW-like halos deviate systematically from the Maxwellian distribution of the SHM. There are more low speed particles and the peak of the distribution is lower and flatter $[115,116]$. Several fitting functions which encapsulate these properties have been proposed $[117,118]$. Hydrodynamical simulations can have different predictions for the local DM distribution depending on their baryonic feedback models, and how well they can reproduce the main galaxy population properties. In particular, some simulations find that the local DM velocity distribution may be better fitted by a Tsallis distribution [119], or have a sharper peak than expected from a Maxwellian distribution [120, 121]. Very recently, however, high resolution hydrodynamic simulations of MW-like galaxies found that the best fit Maxwellian velocity distribution describes well the local DM velocity distributions of simulated haloes [122, 123]. While Ref. [124] found some discrepancy in their results compared to the results of Refs. $[122,123]$, the source of these differences is yet to be understood. Eventually, some anisotropy in the velocity distribution can be expected. It is evaluated by the velocity anisotropy parameter $\beta(r)$ which is a function of the three velocity dispersions along the three Galactic axes $\{x, y, z\}$ and is defined as [99],

$$
\beta(r)=1-\frac{\sigma_{y}^{2}+\sigma_{z}^{2}}{2 \sigma_{x}^{2}} .
$$

At the Solar radius from the Galactic center $\left(R_{\odot}=8 \mathrm{kpc}\right)$, N-body simulations [116, 125-127], with or without baryons, predict a $\beta$ parameter spanning the range 0 to 0.4 which is in favor of radial anisotropy.

In addition to uncertainties in the overall shape of the distribution, the high speed tail contains stochastic features (dubbed "debris flow") from incompletely phase-mixed material [128, 129]. It is also possible that the local DM distribution may contain streams from substructures that are in the process of being disrupted. For instance the Dark Matter component of one of the streams from the Sagittarius dwarf galaxy may pass through the Solar neighbourhood [130, 131].

The baryonic disk can also affect the DM distribution. Late merging halos are dragged into the disk by dynamical friction, where they are destroyed, leading to the formation of a co-rotating dark disk (DD) [132, 133]. While the contribution of the DD to the local DM density is uncertain, it is currently expected to be small, owing to the relatively quiescent merger history of the MW [134]. Eris, a simulation of a MW-like galaxy including baryonic physics, has a DD which contributes less than $10 \%$ of the local DM density [120]. Ref. [122] finds that there may be some hints for the existence of a DD in only 2 out of 14 MW-like galaxies in the EAGLE and APOSTLE hydrodynamic simulations, while Ref. [123] does not find a significant DD in the two MW-like galaxies they studied from the MaGICC simulations. Furthermore, chemo-dynamical analysis of data from the Gaia-ESO Survey has found no evidence for the stellar component of an accreted disk [135]. Further constraints on the dark disk density can be obtained by comparing global and local measures of the DM density (see Sec. 5.4 of Ref. [136] for a review).

In the next section, we show how the velocity distribution enters into the calculation of the directional event rate. In Sec. VIII C we will explore how directional detection may be used to probe the broad characteristics of the DM velocity distribution. In Sec. XI and XII we extend this and discuss the possibility of detecting phase-space substructure and probing more general models for the velocity distribution. For more detailed discussion of astrophysical uncertainties pertaining to direct detection experiments see e.g. Refs. [137, 138]. 


\section{Event rate and cross section}

Directional detection aims at measuring both the energy $\left(E_{r}\right)$ and the $3 \mathrm{D}$ direction $\left(\Omega_{r}\right)$ of a recoiling nucleus ${ }^{\mathrm{A}} \mathrm{X}$ following a WIMP scattering. Since the WIMP speed $v$ in the laboratory rest frame is of the order of $\sim 300 \mathrm{~km} \mathrm{~s}{ }^{-1}$, the recoil energy for elastic scattering is given by the non-relativistic expression

$$
E_{r}=2 v^{2} \frac{\mu^{2}}{m_{A}} \cos ^{2} \theta_{r},
$$

with $\mu=m_{\chi} m_{A} /\left(m_{\chi}+m_{A}\right)$ the WIMP-nucleus reduced mass, $m_{\chi}$ the WIMP mass, $m_{A}$ the mass of the target nucleus and $\theta_{r}$ the angle between the initial WIMP direction $(\hat{\mathbf{v}})$ and that of the recoiling nucleus $(\hat{\mathbf{r}})$, such that $\hat{\mathbf{v}} \cdot \hat{\mathbf{r}}=\cos \theta_{r}$. One expects low energy recoils, typically below $100 \mathrm{keV}$, depending on the particular values of $m_{\chi}$ and $m_{A}$.

The recoil rate per unit detector mass as a function of both the recoil energy and direction is given by the following double-differential spectrum:

$$
\frac{\mathrm{d}^{2} R}{\mathrm{~d} E_{r} \mathrm{~d} \Omega_{r}}=\frac{\rho_{0}}{m_{\chi} m_{A}} \int \frac{\mathrm{d}^{2} \sigma_{\chi}-A}{\mathrm{~d} E_{r} \mathrm{~d} \Omega_{r}} v f(\mathbf{v}) \mathrm{d}^{3} v
$$

with $\rho_{0}$ the local WIMP density, $f(\mathbf{v})$ the WIMP velocity distribution in the detector reference frame and $\mathrm{d}^{2} \sigma_{\chi-A} /\left(\mathrm{d} E_{r} \mathrm{~d} \Omega_{r}\right)$ the double-differential WIMP-nucleus cross section given by :

$$
\frac{\mathrm{d}^{2} \sigma_{\chi-A}}{\mathrm{~d} E_{r} \mathrm{~d} \Omega_{r}}=\frac{\mathrm{d} \sigma_{\chi-A}}{\mathrm{~d} E_{r}} \frac{1}{2 \pi} v \delta\left(\mathbf{v} \cdot \hat{\mathbf{r}}-v_{\min }\right)
$$

where

$$
v_{\min }=\sqrt{E_{r} m_{A} / 2 \mu^{2}}
$$

is the minimum WIMP velocity required to produce a recoil of energy $E_{r}$ in an elastic scattering event. The Dirac delta function originates from Eq. 5 linking the recoil energy and the recoil angle $\theta_{r}$. Note that Eq. 6 assumes that the detector consists of only one nuclide. If it is not the case, the measured rate is given by the sum over all nuclides weighted by the mass fraction.

Under the standard assumption of contact interactions, the WIMP-nucleus cross section is obtained [256] by adding coherently the spin-dependent (SD) and spin-independent (SI) contributions [139]. In the non-relativistic limit, this reads :

$$
\frac{\mathrm{d} \sigma_{\chi-A}}{\mathrm{~d} E_{r}}=\frac{m_{A}}{2 \mu^{2} v^{2}}\left[\sigma^{\mathrm{SI}} F_{\mathrm{SI}}^{2}\left(E_{r}\right)+\sigma^{\mathrm{SD}} F_{\mathrm{SD}}^{2}\left(E_{r}\right)\right],
$$

where $\sigma^{\mathrm{SI}, \mathrm{SD}}$ are the SI and SD WIMP-nucleus cross section at zero momentum transfer and $F_{\mathrm{SI}, \mathrm{SD}}$ the SI and SD form factor.

The double-differential spectrum is given by

$$
\frac{\mathrm{d}^{2} R}{\mathrm{~d} E_{r} \mathrm{~d} \Omega_{r}}=\frac{\rho_{0}}{4 \pi m_{\chi} \mu^{2}}\left[\sigma^{\mathrm{SI}} F_{\mathrm{SI}}^{2}\left(E_{r}\right)+\sigma^{\mathrm{SD}} F_{\mathrm{SD}}^{2}\left(E_{r}\right)\right] \hat{f}\left(v_{\min }, \hat{\mathbf{r}}\right),
$$

where $\hat{f}$ is the three-dimensional Radon transform of the WIMP velocity distribution $f(\mathbf{v})$, given by

$$
\hat{f}\left(v_{\min }, \hat{\mathbf{r}}\right)=\int \delta\left(\mathbf{v} \cdot \hat{\mathbf{r}}-v_{\min }\right) f(\mathbf{v}) \mathrm{d}^{3} v .
$$

Geometrically, the Radon transform is the integral of the function $f(\mathbf{v})$ on a plane orthogonal to the direction $\hat{\mathbf{r}}$ at a distance $v_{\min }$ from the origin. Using the Fourier slice theorem, Gondolo [140] has shown that the use of the Radon transform leads to an analytic evaluation of the event rate for most halo models.

We may then distinguish two types of direct Dark Matter search strategies: directional detection, aiming at measuring the double-differential spectrum, Eq. (10), and direction-insensitive detection, only sensitive to the energy spectrum given by

$$
\frac{\mathrm{d} R}{\mathrm{~d} E_{r}}=\frac{\rho_{0}}{2 m_{\chi} \mu^{2}}\left[\sigma^{\mathrm{SI}} F_{\mathrm{SI}}^{2}\left(E_{r}\right)+\sigma^{\mathrm{SD}} F_{\mathrm{SD}}^{2}\left(E_{r}\right)\right] \int_{v_{\min }} \frac{f(\mathbf{v})}{v} \mathrm{~d}^{3} v .
$$

The event rate, Eqs. (10) and (12), receives inputs from : 
- nuclear physics, via the properties of the target nucleus : $m_{A}, F_{S I, S D}$ as well as the spin content, see Sec. III D

- the particle physics model, via the parameters $m_{\chi}, \sigma^{S I, S D}$, see Sec. IIIE

- the Galactic halo, via the parameters $\rho_{0}$ and $f(\mathbf{v})$, see Sec. III B [141]

The WIMP-nucleus cross section, at zero momentum transfer, can be separated into a SI contribution, given by

$$
\sigma^{\mathrm{SI}}\left({ }^{\mathrm{A}} \mathrm{X}\right)=\frac{4 \mu^{2}}{\pi}\left(\mathrm{Z} f_{p}+(\mathrm{A}-\mathrm{Z}) f_{n}\right)^{2},
$$

where $f_{p, n}$ is the WIMP-proton (resp. neutron) SI coupling constant, and a SD contribution, given by [141]

$$
\sigma^{\mathrm{SD}}\left({ }^{\mathrm{A}} \mathrm{X}\right)=\frac{32}{\pi} G_{F}^{2} \mu^{2} \frac{J+1}{J}\left[a_{p}\left\langle S_{p}\right\rangle+a_{n}\left\langle S_{n}\right\rangle\right]^{2},
$$

where $G_{F}$ is the Fermi constant, $J$ the angular momentum of the target nucleus, $a_{p, n}$ the WIMP-proton (resp. neutron) SD coupling constant, and $\left\langle S_{p, n}\right\rangle$ the spin content of the target nucleus.

For a direct detection experiment to be sensitive to SD interaction, the target nucleus ${ }^{\mathrm{A}} \mathrm{X}$ must have a non-vanishing spin, whereas SI interaction favors heavy targets, due to the $A^{2}$ enhancement. The comparison between various DM detectors, using various target nuclei, must be done at the level of the WIMP-proton (resp. neutron) cross sections, i.e. $\sigma_{p, n}^{\mathrm{SI}} \propto f_{p, n}^{2}$ and $\sigma_{p, n}^{\mathrm{SD}} \propto a_{p, n}^{2}$. It requires either model-dependent assumptions or a dedicated framework valid in the context of heavy squarks [142].

\section{Inputs from nuclear physics}

Two nuclear parameters enter the evaluation of the event rate: the nuclear form factor $F_{S I, S D}$ and the spin content of the target nucleus $\left\langle S_{p, n}\right\rangle$. Although not specific to directional detection, as this issue is shared with direction-insensitive detection, they must be evaluated with caution.

The nuclear form factors $F_{\mathrm{SI}, \mathrm{SD}}(q)$ describes the loss of coherence at high momentum transfer $q=\sqrt{2 m_{A} E_{r}}$. The effect is strong for a heavy WIMP or a heavy target nucleus. Several evaluations of the nuclear form factor are used in the literature, e.g. the Helm form factor [143] or Hartree-Fock (HF) calculations [144], but the differences are small. For SI form factors, it has been shown in Ref. [144] that the differences are $\sim 1 \%$ for a light nucleus at $1 \mathrm{keV}$ and $\sim 10 \%$ for a heavy nucleus at $100 \mathrm{keV}$. The effect on the event rate is only minor for directional detection using $\mathrm{CF}_{4}$. For ${ }^{19} \mathrm{~F}$, the SI (resp. SD) cross section is lowered by $\approx 10 \%$ (resp. $\approx 15 \%$ ) for $E_{r}=100 \mathrm{keV}$ [49], when the form factors are included. Note that uncertainties in the determination of the nuclear form factor $F_{S I, S D}$ will affect the theoretical predictions for the Dark Matter event rate [145].

The spin content of the target nucleus $\left\langle S_{p, n}\right\rangle=\left\langle N\left|S_{p, n}\right| N\right\rangle$ is a key issue for SD detection of Dark Matter. The WIMP couples mainly to the spin of the unpaired proton $\left(e . g .{ }^{19} \mathrm{~F}\right)$ or of the unpaired neutron (e.g. $\left.{ }^{3} \mathrm{He}\right)$. However, this holds true only within the framework of a single-particle shell model. In practice, the spin of the target nucleus is carried both by constituent neutrons and protons. Detailed nuclear shell-model calculations have been developed and the accuracy of the $\left\langle S_{p}\right\rangle$ and $\left\langle S_{n}\right\rangle$ evaluation is assessed by comparing with experimental values (magnetic moments and low energy spectra) [146]. The relative sign of $\left\langle S_{p, n}\right\rangle$ is also important for SD direct detection, inducing either constructive or destructive interference, depending on the sign of the SD amplitudes $a_{p, n}$, see e.g. Ref. [147].

\section{E. Parameters from particle physics models}

WIMP candidates naturally arise from extensions of the standard model of particle physics, e.g. Supersymmetry (SUSY), as long as the lightest particle of the hidden sector, e.g. the lightest supersymmetric particle (LSP), is stable and massive. If its interaction with ordinary matter proceeds only through weak interaction, this particle becomes a good candidate for cold Dark Matter. This is the case for the neutralino which is the LSP in most SUSY models [5].

As long as the WIMP is not discovered at colliders, the particle physics model does not provide inputs for DM searches but only a theoretical framework to interpret the results, within e.g. supersymmetric models. The standard framework assumed for direct detection experiments is in fact inspired by models of supersymmetric DM. It assumes contact interactions (i.e. interactions mediated by heavy particles) and includes only the leading-order interactions 
in the non-relativistic limit. One of the goals of Dark Matter searches, and in particular directional detection, is to constrain the parameters in this framework $\left(m_{\chi}, a_{p}, a_{n}, f_{p}, f_{n}\right)$. The SI WIMP coupling constants with proton and neutron $\left(f_{p}, f_{n}\right)$ are often considered equal, with the assumption that scattering on sea quarks dominates [5]. Hence, the constraint in the SI sector is rather straightforward, provided the SD interaction is neglected. On the other hand, the assumption that one SD interaction dominates must be made in order to constrain either $a_{p}$ or $a_{n}$.

The question of the complementarity between various Dark Matter search strategies, direct detection, indirect detection and colliders, has received much interest in the past years [147-155]. Constraints from all Dark Matter searches, as well as collider [156] and cosmological contraints [1] must be accounted for when studying e.g. the discovery potential of forthcoming detectors or the impact of current results for a given model.

Directional detection aims at constraining both the WIMP (mass and cross section) and halo properties as shown in Refs. [157, 158], see Sec. VIII. Within the framework of neutralino Dark Matter, it has been shown that directional detection is a powerful tool to constrain MSSM and NMSSM parameter spaces [155], as shown in Sec. IX.

\section{F. Non-relativistic effective theory}

Beyond the standard SI and SD interactions, we can also consider interactions which are higher order in the WIMP speed $v$ and recoil momentum $q$. Such a non-relativistic (NR) effective theory approach to WIMP-nucleon elastic scattering was proposed by Fan et al. [159] and extended by others in Refs. [160-162]. Within such a framework, one writes down all Hermitian, Galilean and rotation-invariant interactions composed of the low-energy quantummechanical degrees of freedom of the system. These include the DM and nucleon spins $\mathbf{S}_{\chi}, \mathbf{S}_{N}$ and recoil momentum q. The DM velocity appears only through the Hermitian 'transverse velocity' operator,

$$
\mathbf{v}_{\perp}=\mathbf{v}+\frac{\mathbf{q}}{2 \mu_{n}}
$$

where $\mu_{n}$ is the WIMP-nucleon reduced mass. It is so called because it lies perpendicular to the recoil momentum due to energy conservation, $\mathbf{v}_{\perp} \cdot \mathbf{q}=0$. A full list of such operators can be found in Ref. [160].

For operator $i$, the differential cross-section for WIMP-nucleon scattering can then be written

$$
\frac{\mathrm{d} \sigma}{\mathrm{d} E_{r}}=\frac{1}{32 \pi m_{A} m_{\chi}^{2} v^{2}} \sum_{N, N^{\prime}=p, n} c_{i}^{N} c_{i}^{N^{\prime}} F_{i}^{\left(N, N^{\prime}\right)}\left(q^{2}, v_{\perp}^{2}\right)
$$

Here, $c_{i}^{p, n}$ are the couplings of operator $i$ to protons and neutrons respectively. The nuclear response functions

$F_{i}^{\left(N, N^{\prime}\right)}\left(q^{2}, v_{\perp}^{2}\right)$ are composed of powers of $q^{2}$ and $v_{\perp}^{2}$ multiplied by generalised nuclear form factors. These form factors have been obtained numerically for a variety of target nuclei, see e.g. Refs. [162, 163]. NR operators which are typically studied lead to nuclear response functions proportional to $\left(v_{\perp}\right)^{0}$ (which is the standard case) or proportional to $\left(v_{\perp}\right)^{2}$. In the latter case, the integral over $\mathbf{v}$ leads to the 'transverse' Radon Transform, given by:

$$
\hat{f}^{T}\left(v_{\min }, \hat{\mathbf{r}}\right)=\int\left(v^{2}-(\mathbf{v} \cdot \hat{\mathbf{r}})^{2}\right) f(\mathbf{v}) \delta\left(\mathbf{v} \cdot \hat{\mathbf{r}}-v_{\min }\right) \mathrm{d}^{3} \mathbf{v}=\int\left(v^{2}-v_{\min }^{2}\right) f(\mathbf{v}) \delta\left(\mathbf{v} \cdot \hat{\mathbf{r}}-v_{\min }\right) \mathrm{d}^{3} \mathbf{v} .
$$

This NR effective theory framework has been used to interpret recent data from direction-insensitive detection ( $e . g$. Ref. [164]) and its impact in directional detectors has been studied in Refs. [165, 166]. In Ref. [166], directional rates $\mathrm{d} R / \mathrm{d} \cos \theta$ were studied for 14 different $\mathrm{NR}$ operators for detectors composed of $\mathrm{CS}_{2}, \mathrm{CF}_{4}$ and ${ }^{3} \mathrm{He}$. This study revealed that the contribution of the operator $\mathcal{O}_{11}=i \mathbf{S}_{\chi} \cdot \mathbf{q} / m_{N}$, where $m_{N}$ is the nucleon mass, to the scattering rate in $\mathrm{CF}_{4}$ can be comparable to the standard SI and SD interactions, despite being suppressed by powers of the recoil momentum. Ref. [165] compared the directionality of the signal for a number of NR operators, assuming equal numbers of events for each operator. The resulting signals may be more strongly directional (for operators coupling to $\mathbf{q}$ ) or more isotropic (for operators coupling to $\mathbf{v}_{\perp}$ ) than in the standard SI/SD scenario. In Sec. IX C, we discuss how directional detection may be used to discriminate between different operators within the NR effective theory framework.

\section{DIRECTIONAL FEATURES}

\section{A. Introduction}

The main observable signature for directional detectors is the dipole in Galactic coordinates of the directions of the WIMP-induced nuclear recoils. In section IV B, we describe this dipole feature. We also review two other observable 


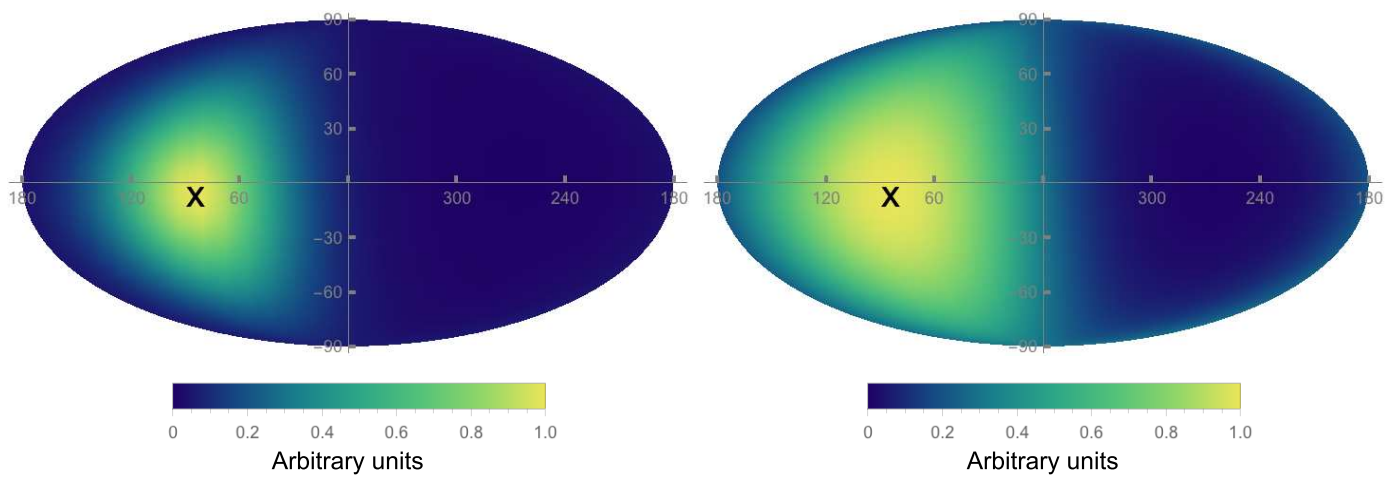

FIG. 1: Left: Flux of $100 \mathrm{GeV}$ WIMPs moving with speeds higher than $v_{\min }$ as needed to produce $25 \mathrm{keV}$ F recoils. Right: Angular distribution of the energy differential recoil rate in F for WIMP mass $100 \mathrm{GeV}$, and recoil energy of $25 \mathrm{keV}$. Maps are incoming direction of WIMP-induced recoils in Mollweide equal-area projections, in Galactic coordinates. For convenience, we present the direction of $\mathbf{v}_{\text {lab }}$ as a cross on the maps.

signatures unique to directional detectors in sections IV C and IV D: the ring and aberration features. The detection of ring and aberration features requires lower energy thresholds and more events than the detection of the dipole, but they can provide additional constraints on the WIMP and halo properties, see Sec. VII.

The Radon transform (Eq. 11) in the laboratory frame for the truncated Maxwellian WIMP velocity distribution, Eq. 3, is [140]

$$
\hat{f}\left(v_{\min }, \hat{\mathbf{r}}\right)= \begin{cases}\frac{1}{N_{\mathrm{esc}}\left(2 \pi \sigma_{v}^{2}\right)^{1 / 2}}\left\{\exp \left[-\frac{\left[v_{\min }+\hat{\mathbf{r}} \cdot \mathbf{v}_{\mathrm{lab}}\right]^{2}}{2 \sigma_{v}^{2}}\right]-\exp \left[-\frac{v_{\mathrm{esc}}^{2}}{2 \sigma_{v}^{2}}\right]\right\} & \text { if } v_{\mathrm{min}}+\hat{\mathbf{r}} \cdot \mathbf{v}_{\mathrm{lab}}<v_{\mathrm{esc}} \\ 0 & \text { otherwise }\end{cases}
$$

Here $\mathbf{v}_{\text {lab }}$ is the velocity of the laboratory with respect to the Galaxy (hence the average velocity of the WIMPs with respect to the detector is $-\mathbf{v}_{\text {lab }}$ ), and

$$
N_{\mathrm{esc}}=\operatorname{erf}\left(\frac{v_{\mathrm{esc}}}{\sqrt{2} \sigma_{v}}\right)-\sqrt{\frac{2}{\pi}} \frac{v_{\mathrm{esc}}}{\sigma_{v}} \exp \left[-\frac{v_{\mathrm{esc}}^{2}}{2 \sigma_{v}^{2}}\right]
$$

The nuclear recoil direction $\hat{\mathbf{r}}$ is measured in the detector reference frame, and in order to compute $\hat{f}$ we need to evaluate $\hat{\mathbf{r}} \cdot \mathbf{v}_{\text {lab }}$. The transformation equations for $\hat{\mathbf{r}}$ and $\mathbf{v}_{\text {lab }}$ to go from the detector frame to the Galactic reference frame are given in Appendix A.

One can see from Eq. 18 that there are two regimes of interest, depending on the value of $v_{\text {min }}$, as defined by Eq. 8 . First, if $v_{\min }>v_{\text {lab }}$, then the argument of the first exponential cannot be zero, but is minimized when $\hat{\mathbf{r}}$ and $\mathbf{v}_{\text {lab }}$ are anti-parallel. This leads to a dipole feature in the recoil angle distribution (Sec. IV B). Second, if $v_{\min }<v_{\text {lab }}$, i.e. for low recoil energies and large WIMP masses (see Eq. 8), then the argument of the first exponential can be zero, and the recoil angle distribution will exhibit a ring-like feature (see Sec. IV C).

\section{B. Dipole feature}

A directional detector located on the Earth will experience a WIMP head-wind caused by the Earth's motion through the Galactic WIMP distribution (the halo). The resulting WIMP-induced nuclear recoils will come from the direction to which the vector $\mathbf{v}_{\text {lab }}$ is pointing. This dipole feature was first described by Spergel [17], who showed that the recoil rates in the forward and backward directions differed by a factor of order 10, depending on the recoil energy threshold. Because no known backgrounds can mimic this angular signature, the dipole feature, which is only accessible to directional detectors, is generally considered to be a smoking-gun evidence for WIMP Dark Matter.

As an example of the dipole feature, Figure 1 left shows a map of the WIMP flux in Galactic coordinates, assuming that the WIMP velocity distribution is Maxwellian. The incoming WIMP flux appears to come primarily from the direction of the Earth's motion through the Galaxy, shown as cross at position $(l, b)=(\pi / 2,0)$, where $(l, b)$ are Galactic longitude and latitude. Figure 1 right presents the incoming direction of WIMP-induced recoils (for 


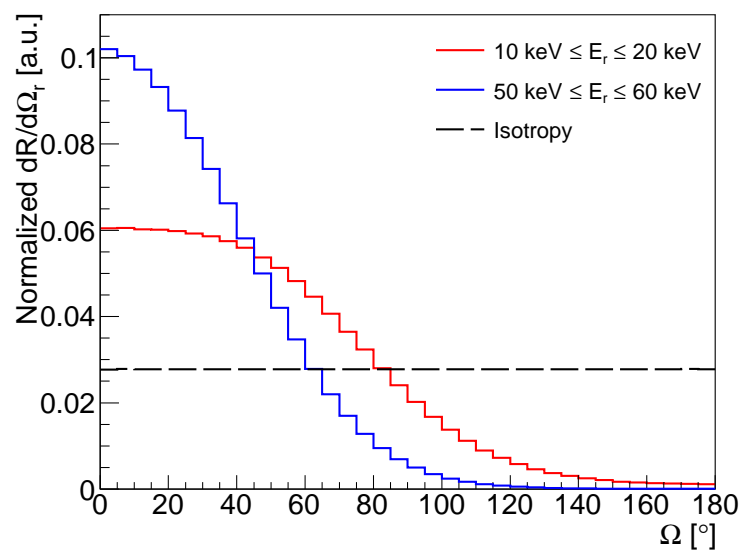

FIG. 2: Normalized angular spectra for F recoils in two energy ranges: $10-20 \mathrm{keV}$ and 50-60 keV for a WIMP mass of $100 \mathrm{GeV}$. Here, $\Omega$ is the opening angle between the recoil direction and the lab velocity vector. The dipole signature is stronger for higher energy recoils.

$\left.E_{r}=25 \mathrm{keV}\right)$ in galactic coordinates. Note that in this review, the chosen convention is to present recoil maps as directions where the recoils originate from, in the spirit of WIMP astronomy. For convenience, the cross on the map presents the direction of $\mathbf{v}_{\text {lab }}$, to highlight the fact that WIMPs are coming from the direction to which the laboratory's velocity vector is pointing to, which happens to be roughly in the direction of the constellation Cygnus $(l, b)=(\pi / 2,0)$. In Figure 2, we take advantage of the azimuthal symmetry of the recoil directions about $\mathbf{v}_{\text {lab }}$ to plot the fraction of recoils per solid angle as a function of the angle between the recoil direction $\hat{\mathbf{r}}$ and $-\hat{\mathbf{v}}_{\text {lab }}$. From these normalized angular spectra, we see that higher recoil energies lead to a tighter clustering of recoil angles, and therefore a stronger dipole signature, but at the expense of a smaller event rate. It is also clear that only modest angular resolution (tens of degrees) is required to resolve the dipole signature.

Due to the non-zero energy threshold, the WIMP induced recoil angular distribution does also depend on the target mass $m_{A}$. As several directional detectors are being developed with different target nuclei, we discuss the influence of these targets. Although their detection characteristics may be different (e.g. track length, drift velocity and straggling, see Sec. II), for low mass targets $\left({ }^{12} \mathrm{C},{ }^{32} \mathrm{~S},{ }^{19} \mathrm{~F}\right)$ and at sufficiently low recoil energy (when the form factor can be approximated to unity), equivalent directional signal can be found by adjusting the energy range for each target. Indeed, the directionality of the signal is encoded only in the $\cos \theta_{r}$ term of Eq. (5), then the angular distribution for a target of mass $m_{A_{1}}$ at a recoil energy $E_{r_{1}}$ is equivalent to the one of a $m_{A_{2}}$ target at $E_{r_{2}}$, using :

$$
E_{r_{2}}=E_{r_{1}} \frac{m_{A_{2}}}{m_{A_{1}}}\left(\frac{m_{\chi}+m_{A_{1}}}{m_{\chi}+m_{A_{2}}}\right)^{2}
$$

Therefore, if we consider a $10 \mathrm{GeV}$ WIMP mass, equivalent directional distributions are expected from a ${ }^{19} \mathrm{~F}$ target with an energy range from $5 \mathrm{keV}$ to $50 \mathrm{keV}$ and from a ${ }^{32} \mathrm{~S}$ target with an energy range from $4 \mathrm{keV}$ and $40 \mathrm{keV}$. As a matter of fact, as long as the form factor can be approximated to unity (small momentum transfer), if we consider a recoil energy range from $0 \mathrm{keV}$ to infinity, the angular distribution of the WIMP induced recoils are independent of the WIMP mass and the target nucleus. Hence, all results (skymaps, angular spectra) presented hereafter for a given target nucleus may be converted to another one by using Eq. 20.

\section{Ring-like features}

An additional Dark Matter signature that can be searched for in directional detectors is a ring-like feature in the recoil rate. For large WIMP masses and low recoil energies, the recoil rate is maximum in a ring around the average WIMP arrival direction. For example, if the WIMP mass is $100 \mathrm{GeV}$, then the recoils of ${ }^{19} \mathrm{~F}$ will exhibit a ring-like pattern (rather than a dipole) for recoil energies below $14 \mathrm{keV}$. If the WIMP mass is $10 \mathrm{GeV}$, then the ring-dipole transition energy is even smaller $(2.4 \mathrm{keV})$. The cause of the ring-like geometry is most easily seen in the expression of the Radon transform (see Eq. 18). In that case, if $v_{\min }<v_{\text {lab }}$, then the maximum of $\hat{f}\left(v_{\min }, \hat{\mathbf{r}}\right)$ occurs when 
$-\hat{\mathbf{r}} \cdot \mathbf{v}_{\text {lab }}=v_{\min }$, or at an angle $\gamma$ between $\hat{\mathbf{r}}$ and $-\mathbf{v}_{\text {lab }}$ given by

$$
\cos \gamma=\frac{v_{\text {min }}}{v_{\text {lab }}}=\sqrt{\frac{m_{A} E_{r}}{2 \mu^{2} v_{\text {lab }}^{2}}} .
$$

The angular radius of the ring, $\gamma$, is larger for larger values of $v_{\text {lab }}$, and can reach $90^{\circ}$ [167].

The main uncertainty in $v_{\text {lab }}$ is from the uncertainty in the Galactic rotation speed, $v_{c}$ (see Appendix A for the relation between $\mathbf{v}_{c}$ and $\mathbf{v}_{\text {lab }}$ ). As mentioned in Sec. III B, there is a wide range of values for $v_{c}$ depending on the model used for the rotation curve. In particular Ref. [98] found values ranging from $v_{c}=(200 \pm 20) \mathrm{km} \mathrm{s}^{-1}$ to $v_{c}=(279 \pm 33) \mathrm{km} \mathrm{s}^{-1}$. In this Section and in Sec. VII we take $v_{c}=180 \mathrm{~km} \mathrm{~s}^{-1}$ and $312 \mathrm{~km} \mathrm{~s}{ }^{-1}$ as low and high estimates. Notice also that the times of maximum and minimum $v_{\text {lab }}$ depend on the value of $v_{c}$, and are not exactly half a year apart due to the ellipticity of the Earth's orbit.

The ring-like feature depends on $\hat{f}$, and the equations presented here refer to the case of WIMP-nucleus elastic scattering. The ring-like feature would be different for WIMP interaction types which modify the kinematics of the scattering (e.g. inelastic interaction) or change the form of the Radon transform $\hat{f}$ (Eq. 18). To give examples of how the ring would appear in an actual recoil rate, in Fig. 3 we show Mollweide equal-area projection maps of the incoming differential recoil rate in Galactic coordinates, assuming WIMPs with elastic SD interactions in a Fluorine detector, for $E_{r}=5 \mathrm{keV}, m_{\chi}=100 \mathrm{GeV}, v_{\mathrm{esc}}=544 \mathrm{~km} \mathrm{~s}^{-1}$, and four combinations of $v_{\text {lab }}$ and $\sigma_{v}$. For convenience, we present the direction of $\mathbf{v}_{\text {lab }}$ as a cross on the map. The ring of maximum recoil rate is clearly seen in all four panels of Fig. 3, but is most visible in the bottom right panel which has the smallest $\sigma_{v}$ and largest $v_{\text {lab }}$ combination of the four.

The ring is easiest to see when the rate at the ring has the largest contrast with respect to the rate at the center of the ring $((l, b)=(\pi / 2,0))$. The ratio of the Radon transform at the center of the ring, $\hat{f}_{\text {center }}$, to the Radon transform at the ring, $\hat{f}_{\text {ring }}$, is approximately, $\hat{f}_{\text {center }} / \hat{f}_{\text {ring }} \simeq \exp \left[-\left(v_{\text {lab }}-v_{\text {min }}\right)^{2} / 2 \sigma_{v}^{2}\right]$. Hence, the best chance to observe the ring is for large WIMP masses and low recoil energies (such that $v_{\text {min }}$ is small), small $\sigma_{v}$, and large $v_{\text {lab }}$. We discuss the prospects of observing the ring-like feature in Sec. VII B.

We note that an enhanced ring-like feature may appear for certain non-relativistic operators [165, 166] described in Sec. III F. For operators coupling to $\mathbf{v}_{\perp}$, the recoil spectrum is governed by the transverse Radon Transform $\hat{f}^{T}\left(v_{\min }, \hat{\mathbf{r}}\right)$ (Eq. III F) which leads to a suppression of the rate when the incoming Dark Matter velocity is parallel to the recoil direction. This suppression leads to an enhanced ring, which is observable for larger values of $v_{\text {min }}$, up to $v_{\text {min }} \approx 2 v_{\text {lab }}$. For these NR operators, then, a ring-like feature may be visible for WIMP masses as low as $20-30 \mathrm{GeV}$ or for energy thresholds up to $E_{\mathrm{th}} \sim 20 \mathrm{keV}$ for heavier WIMPs [165].

\section{Aberration features}

Aberration features in a Dark Matter signal are changes in the recoil direction pattern, due to the change in the direction of the arrival velocity of WIMPs on Earth caused by Earth's motion around the Sun [168]. Due to Earth's revolution around the Sun, the mean recoil direction will change with a period of one year. Aberration features depend on the velocity of the Earth's revolution around the Sun and the local WIMP velocity distribution. Knowing the former, such features can be used to determine the latter.

The annual change in the magnitude of $\mathbf{v}_{\text {lab }}$ due to the motion of the Earth around the Sun causes the nondirectional annual modulation of the rate [13]. Furthermore, the change in both magnitude and direction of $\mathbf{v}_{\text {lab }}$ during the year causes the aberration features. As with the ring-like features, the aberration features depend on the Radon transform $\hat{f}$. Let us consider the annual variation of the directional differential recoil rate,

$$
\Delta\left(\frac{\mathrm{d}^{2} R}{\mathrm{~d} E_{r} \mathrm{~d} \Omega_{r}}\right)=\frac{\mathrm{d}^{2} R_{2}}{\mathrm{~d} E_{r} \mathrm{~d} \Omega_{r}}-\frac{\mathrm{d}^{2} R_{1}}{\mathrm{~d} E_{r} \mathrm{~d} \Omega_{r}},
$$

where the subscripts " 1 " and " 2 " refer to the times in a year when $v_{\text {lab }}$ is minimum and maximum, respectively. To visualize the aberration patterns, one can plot this annual variation in a Mollweide map of the incoming recoil directions in Galactic coordinates.

To give specific examples, we choose WIMPs with elastic SD interactions in a Fluorine detector. Fig. 4 shows the directional differential recoil rate in $\mathrm{F}$ at $E_{r}=5 \mathrm{keV}$ and assuming $v_{c}=180 \mathrm{~km} \mathrm{~s}^{-1}$ on December 1 (left panel) when $v_{\text {lab }}$ is minimum, and May 30 (center panel) when $v_{\text {lab }}$ is maximum, plotted in Mollweide maps of the incoming recoil directions in Galactic coordinates for a $100 \mathrm{GeV}$ WIMP. The cross indicates the direction of $\mathbf{v}_{\text {lab }}$. The difference between the December and May maps in the left and center panels is shown in the right panel, where a characteristic aberration pattern is visible. 

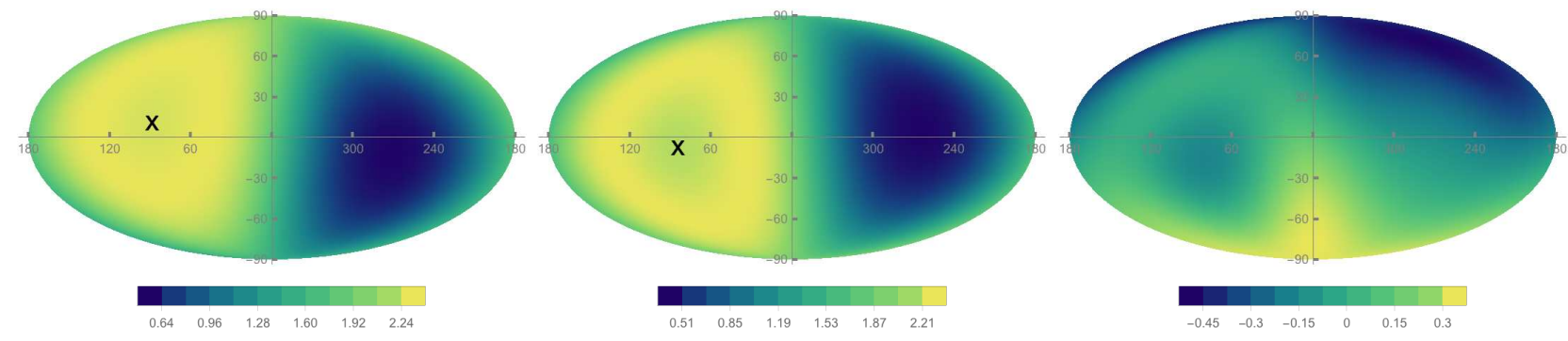

FIG. 4: Directional differential recoil rate at $E_{r}=5 \mathrm{keV}$ in F for a WIMP of mass $m_{\chi}=100 \mathrm{GeV}$. We assume $v_{c}=180 \mathrm{~km}$ $\mathrm{s}^{-1}, \sigma_{v}=173 \mathrm{~km} \mathrm{~s}^{-1}$ and $v_{\mathrm{esc}}=544 \mathrm{~km} \mathrm{~s}^{-1}$. Left panel: on December 1. Center panel: on May 30 (same as the top right panel of Fig. 3). For convenience, we present the direction of $\mathbf{v}_{\text {lab }}$ as a cross on the map (in the NGH in December and in the SGH in May). Right panel: May - Dec difference of the directional differential recoil rates. The values corresponding to each color in the horizontal bars are given in units of $10^{-5} \times\left(\rho_{0.3} \sigma_{p, 40}^{\mathrm{SD}} / \mathrm{kg}\right.$ day $\left.\mathrm{keV} \mathrm{sr}\right)$.

signal and background. This is generally true for any background which has an angular distribution that differs from that of the WIMP recoil signal, however the advantage is perhaps most striking when the background itself has strong directional features, as is the case for Solar neutrino-induced recoils. This particular background is irreducible event-by-event except by directionality. As a result, directional detectors can set stronger exclusion limits than nondirectional detectors, for equal exposures. Below, we discuss several statistical methods that have been developed to set improved limits with directional detectors, by incorporating both energy and angular information.

\section{B. Maximum Patch Method}

The Maximum Gap Method [173] has been used to set limits in non-directional Dark Matter experiments when the background distribution is not a priori known. The basic idea is straightforward: the largest range of energy where no events have been recorded (i.e. the maximum energy gap) is used to calculate the cross section limit. The method thus automatically excludes energy regions where unexpected backgrounds may be found after unblinding a data sample, and the corresponding statistical penalty factor is taken into account. The limit calculation assumes the theoretical prediction for the one-dimensional energy spectrum as the signal probability density function (PDF), and uses the number of events and resulting gap sizes measured in experimental data. The Maximum Patch Method [174] generalizes the maximum gap method to two dimensions, by using the double-differential spectrum as the signal PDF and looking for an event-free 'patch' in energy-recoil-angle space. As expected, pseudo experiments show that the maximum patch method with data from a directional detector is more sensitive than the maximum gap method with a non-directional detector. Both outperform the traditional Poisson method, which counts all events observed as signal candidates and lacks knowledge of the expected signal shape.

The improvement seen with the maximum patch procedure is most dramatic when the number of background events is large, and when the WIMP mass is low. The former because including the angle dimension can improve sensitivity for a fixed energy window (as would be selected by the maximum gap method using energy only), and the latter because of the relatively steeper shape of the recoil angle versus energy distribution for low versus high masses. This method can result in a limit that is up to one order of magnitude improved relative to the energy-only maximum gap case. Ref. [174], however, cautions that the quantitative degree of improvement depends on the distribution of measured events. Indeed, the limit set using this method can be either better or worse than the energy-only limit, depending on the background distribution and number of background events. This occurs because the maximum gap (and patch) methods restrict the experiment's acceptance to the fraction of parameter space in which there are zero events. If this gap (or patch) is very small, or happens to exclude the regions of energy and/or angle parameter space that have the strongest parameter dependence of the signal distribution, then the resulting sensitivity can be worse than for an experiment with a larger acceptance (e.g. a lower energy threshold) with a small population of background events. Reference [173] shows that this can be ameliorated by choosing the "optimum" (rather than maximum) gap, which is the region of parameter space that maximizes sensitivity including a non-zero number of background events. This is generally true for both directional and non-directional searches.

This maximum patch method is general, and can be used for any combination of $n$ variables with discriminating power to distinguish signal from background. The maximum patch method has been employed beyond directional searches [175] to search for inelastic dark matter in the parameter space of energy and particle identification. In the analysis of Ref. [175], the resulting sensitivity is significantly improved relative to an energy-only analysis. 
it has a factor of 2-3 effect on exclusion limits. The effect of detector angular resolution on directional exclusion limits is also similar. The detector energy threshold is of primary importance, in particular for low WIMP masses, as shown in Fig. 5 (right). The effect of astrophysical uncertainties are estimated by varying the asymptotic circular velocity $v_{c}$ from $170 \mathrm{~km} \mathrm{~s}^{-1}$ to $270 \mathrm{~km} \mathrm{~s}^{-1}$, compared to the standard value of $220 \mathrm{~km} \mathrm{~s}^{-1}$. This has a small (20\%-50\%) effect on exclusion limits, where the largest effects are seen for low-mass $(10 \mathrm{GeV})$ WIMPs.

Ref. [176] presents expected limits using the likelihood method for a prototype detector as proposed by the MIMAC collaboration [26]. Given a 10-kg-year exposure, $10^{\circ}$ angular resolution, and a recoil energy range of $5-50 \mathrm{keV}$, such a detector would be able to exclude spin-dependent WIMP-proton scattering down to cross sections of $2 \times 10^{-6} \mathrm{pb}$ for background free running. With backgrounds at the rate of $10 / \mathrm{kg} /$ year, cross sections down to $10^{-4} \mathrm{pb}$ can be excluded by a detector without sense recognition. With sense recognition, the limits improve by a factor of 2-3. These limits are better than experimental spin-dependent limits at the time of writing, though the field is changing quickly.

\section{D. $\chi^{2}$ Test}

The NEWAGE collaboration have developed a method based on a $\chi^{2}$ test to set dark matter limits incorporating both energy and directional information [27]. In fact this is the only published limit from a directional experiment that uses the measured direction in the limit setting procedure. In this analysis the 3D track axial direction is measured, and therefore the $\cos \theta$ distribution is folded into $|\cos \theta|$, where $\theta$ is the measured recoil angle relative to the direction to Cygnus at the time of the event. The limit-setting procedure performs a $\chi^{2}$ test between the measured and predicted $|\cos \theta|$ distributions at each dark matter mass, in bins of recoil energy, varying the Dark Matter WIMP-nucleon cross section in order to determine the $90 \%$ confidence level value of the cross section.

To produce the $|\cos \theta|$ predicted distribution, the differential rate of recoil events as a function of energy $E_{r}$ and angle $\cos \theta, \frac{d R}{d E_{r} d(\cos \theta)}$ is calculated for each dark matter mass. To generate the angles, the simulation samples the direction to Cygnus over the live time of the detector. The simulation accounts for the quenching of the energy loss to convert $E_{r}$ into visible energy $E_{v i s}$ measured in electron-equivalent $\mathrm{keV}$ (keVee), smears the event by the measured angular and energy resolutions, and weights the event by the detection efficiency, which is a function of the energy and track angle in the local detector coordinates. In this way, the simulation produces a $|\cos \theta|$ distribution, in bins of visible energy, that can be compared with the measured $|\cos \theta|$ distribution.

A $\chi^{2}$ test is performed between the measured and predicted $|\cos \theta|$ distributions in order to set a limit, in a raster scan over candidate dark matter masses. All detected nuclear recoil events passing cuts are considered as signal WIMP events, with no background subtraction. Given the angular resolution ( $55^{\circ}$ at $\left.100 \mathrm{keVee}\right)$ and the statistics, the $\chi^{2}$ fit is performed between measured and predicted distributions in two-bin $|\cos \theta|$ distributions in order to find the best-fit cross section for a given dark matter mass, for each of 15 energy bins spanning the range 100-400 keVee independently.

As presented in Ref. [27], the best fit cross section is $5500 \mathrm{pb}$ at $100 \mathrm{GeV}$, with a $\chi^{2}$ per degree of freedom of 3.7, and the most stringent resulting $90 \%$ confidence level limit is $5400 \mathrm{pb}$ at $150 \mathrm{GeV}$. This procedure is also used to test the compatibility of the data with an isotropic angular distribution. The $\chi^{2}$ per degree of freedom in this case is 0.11 . In this way, the NEWAGE experiment have used the $\chi^{2}$ test on the measured $|\cos \theta|$ distribution to disfavour the dark matter hypothesis relative to the presence of an isotropic background.

\section{DIRECTIONAL DISCOVERY}

\section{A. Introduction}

In the previous section we discussed the power of directional detection to set limits on the WIMP-nucleon interactions. In this section, we consider how directional detection can be used to confirm the WIMP origin of recoil events, once s significant excess has been observed.

The directional recoil rate depends on both of the angles which specify a given direction, however the strongest signal is the event rate with respect to the angle between the recoil direction and the direction of Solar motion [17, 178]. Spergel found that the event rate in the forward direction is up to an order of magnitude larger than that in the backward direction [17]. Copi and Krauss showed that consequently a WIMP signal could be distinguished from backgrounds with as few as 30 events [179].

The WIMP search strategy for a directional detector can be divided into two phases. The first step is to check that the recoils are anisotropic, and hence unlikely to be due to backgrounds, see Sec. VIB. The next step is to measure the mean recoil direction and check that it coincides with the direction of Solar motion [257], see Sec. VIC. This 
would provide robust confirmation of the Galactic origin of the recoil events. In Sec. VIE we discuss how directionality can overcome the neutrino floor.

\section{B. Detecting anisotropy}

Most of the background contributions to directional detectors are expected to have distributions that are close to isotropic in the Galactic frame. Therefore a detection of anisotropy would strongly suggest a Dark Matter origin for (at least some of) the recoils. Copi and Krauss first investigated such detection of anisotropy within a model-dependent approach, i.e. taking into account the expected angular distributions for both the WIMP and background induced nuclear recoils $[179,180]$. Using an unbinned likelihood analysis they showed that, for plausible halo models, the angular distribution of WIMP induced recoils could be discriminated from isotropic backgrounds with a reasonable number of events, depending on the background contamination and the energy threshold of the experiment considered. For example, they found that only 50 events would be required to reject the isotropy hypothesis in the case of the Standard Halo Model and a WIMP fraction of $\lambda=0.5$.

Morgan, Green and Spooner studied non-parametric spherical statistics for detecting anisotropy [70]. These nonparametric statistics have the advantage of not requiring any assumptions to be made about the direction dependence of the WIMP recoil rate (or equivalently the WIMP velocity distribution). As the nuclear recoils induced by WIMPs are expected to exhibit a dipole-like angular distribution, the most powerful test for rejecting isotropy uses the mean angle between the observed recoil directions and the direction of motion of the Sun:

$$
\langle\cos \theta\rangle=\frac{\sum_{i=1}^{N} \cos \theta_{i}}{N},
$$

where $\theta_{i}$ is the $3 \mathrm{~d}$ angle between the direction of Solar motion and the $i$-th recoil vector and $N$ is the number of events. For isotropic recoils $\langle\cos \theta\rangle$ can take values in the range $[-1,1]$ and, due to the central limit theorem, for a sufficiently large number of events $\langle\cos \theta\rangle$ approaches a Gaussian distribution with mean zero. On the other hand for WIMP induced recoils $\langle\cos \theta\rangle$ will be positive, due to the concentration of the WIMP flux around the direction of Solar motion. Using this statistic an ideal detector, with zero background, which can measure the sense $(+\hat{\mathbf{r}}$ vs. $-\hat{\mathbf{r}})$ of nuclear recoils in 3d with good angular resolution would require only 10 events to reject isotropy at $95 \%$ confidence. With such a model-independent method the number of events required to reject isotropy is highly sensitive to the background contamination as it is not subtracted. For WIMP fractions $\lambda=0.5$ and 0.09 , the number of WIMP induced events required increases to 27 and 170 respectively.

\section{Measuring the mean recoil direction}

Once anisotropy of the nuclear recoil distribution has been detected, the Galactic origin of the observed events can be established by measuring the mean recoil direction. If the mean recoil direction is found to be consistent, within a few degrees, with the direction of motion of the Sun, $\left(l_{\odot}, b_{\odot}\right)$ in Galactic coordinates, then Dark Matter will be unambiguously discovered.

Green and Morgan proposed using the median recoil direction to probe the mean recoil direction [181]. The median direction is defined [182] as the direction, $\hat{\mathbf{r}}_{\text {med }}$, which minimises the sum

$$
\mathcal{M}=\sum_{i=1}^{N} \cos ^{-1}\left(\hat{\mathbf{r}}_{\text {med }} \cdot \hat{\mathbf{r}}_{i}\right),
$$

where $\hat{\mathbf{r}}_{i}$ are the recoil directions. They found that with $\sim 30$ events an ideal detector could demonstrate that the median recoil direction coincides with the direction of Solar motion at $95 \%$ confidence. As discussed above, as backgrounds are not subtracted in this model-independent approach, non-zero isotropic backgrounds would increase this number, significantly if the signal is subdominant. For instance for $\lambda=0.1$ roughly 300 events would be required.

Billard et al. [71] investigated measuring the peak recoil direction with a map-based likelihood method using Poisson statistics:

$$
\mathscr{L}\left(m_{\chi}, \lambda, \ell, b\right)=\prod_{i=1}^{N_{\text {pixels }}} P\left(\left[(1-\lambda) B_{i}+\lambda S_{i}\left(m_{\chi} ; \ell, b\right)\right] \mid M_{i}\right),
$$

where $N_{\text {pixels }}$ is the number of pixels, $B_{i}, S_{i}$ and $M_{i}$ are the background, WIMP-induced and observed recoil rates in each pixel respectively. The likelihood analysis has four free parameters: the WIMP mass, $m_{\chi}$, the WIMP fraction, 


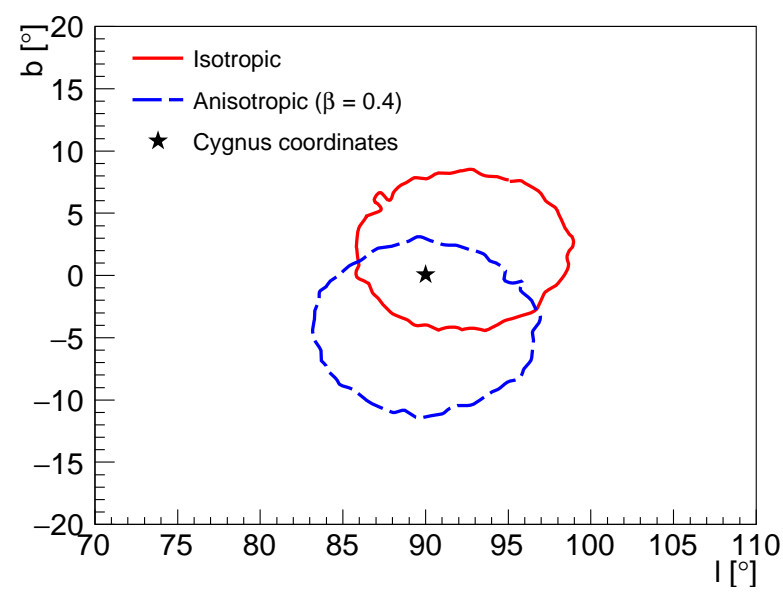

FIG. 6: $95 \%$ contour level in the $\left(\ell_{\odot}, b_{\odot}\right)$ plan for two input halo models: Isotropic (red solid line) and radially anisotropic with $\beta=0.4$ (blue solid line), see Eq. 4 for a definition of $\beta$. These results have been obtained for a 50 GeV WIMP mass with a $10^{-3}$ pb SD-p cross section, a $10 \mathrm{~kg} \mathrm{CF}_{4}$ directional detector with an energy threshold of $5 \mathrm{keV}, 3$ years of exposition time and a flat background component of 10 events $/ \mathrm{kg} /$ year. Figure adapted from Ref. [157].

$\lambda$ (see Eq. 2), and the Galactic coordinates of the direction in which the WIMP directional rate is maximum, $(\ell, b)$. Hence, $S\left(m_{\chi} ; \ell, b\right)$ corresponds to a rotation of the $S\left(m_{\chi}\right)$ distribution by the angles $\left(\ell^{\prime}=\ell-\ell_{\odot}, b^{\prime}=b-b_{\odot}\right)$. They used $N_{\text {pixels }}=768$ to take into account the rather low $\left(15^{\circ} \mathrm{FWHM}\right)$ angular resolution of the recoil direction. As well as measuring the peak direction of the observed recoil distribution, and checking its consistency with the direction of Solar motion, this approach allows the background contribution to be subtracted so that the fraction of WIMP events, and therefore the WIMP-nucleon cross section, can be constrained as a function of the WIMP mass. They found that, assuming $3 \mathrm{~d}$ read-out and sense recognition, the peak direction could be confirmed to be within $20^{\circ}$ degrees of the direction of Solar motion, with as few as 25 WIMP events, even with non-negligible backgrounds. In fact even for a WIMP fraction as low as $\lambda=0.1$ the uncertainty in the peak direction is only increased by a factor of two, which demonstrates the robustness of this approach to background contamination of the data. Finally, this approach also allows the Bayesian significance of a potential WIMP signal to be estimated. For $\lambda=1 / 3$ only 25 WIMP events are required to reach the $3 \sigma$ significance required for the discovery of Dark Matter. In Ref. [183], Billard et al. extended this analysis technique to non-standard halo models and confirmed that recovering the main incoming direction of the event direction is a robust signature of a positive DM detection. Indeed, Fig. 6 shows two 95\% C.L. contours in the $(\ell, b)$ plane derived from the likelihood analyses of two different data sets comprising 300 WIMP events and 1000 background events but considering two different halo models: isotropic (red contour) and strongly radially anisotropic with $\beta=0.4$ (blue contour), see Eq. 4 for a definition of $\beta$. In both cases, the main incoming direction of the WIMP events are fully consistent with the direction of the Solar motion.

As discussed in Sec. III F, considering more general WIMP-nucleon interactions may affect the directionality of the signal and therefore the number of events required to reject isotropy or confirm the median recoil direction. Using the framework of non-relativistic (NR) effective theory, Ref. [165] concludes that there may be roughly a factor of 2 uncertainty on the number of events required, owing to a lack of knowledge about which operator mediates the interaction.

\section{Experimental considerations}

Regardless of the specific data analysis techniques used, the directional energy threshold plays an important role in determining the number of events, and hence exposure, required to detect an anisotropic WIMP signal. The high energy events are more anisotropic, therefore as the energy threshold is increased the number of events required decreases, however the event rate above threshold also decreases significantly. In fact only if the energy threshold is extremely low is it possible to decrease the exposure required by slightly increasing the energy threshold of the experiment [184].

Green and Morgan investigated how the number of events required to reject isotropy using model independent statistics depends on the detector capabilities, in particular whether the recoil vectors are measured in two or three 
dimensions and whether or not the senses of the recoils can be measured [184]. If the recoil senses are not measured, i.e. the data is axial rather than vectorial, then the test statistic, Eq.(23), is modified to

$$
\langle|\cos \theta|\rangle=\frac{\sum_{i=1}^{N}\left|\cos \theta_{i}\right|}{N},
$$

and in this case for isotropic backgrounds $\langle|\cos \theta|\rangle$ takes values in the range $[0,1]$ with a mean, in the large $N$ limit, of 0.5. For $2 \mathrm{~d}$ read-out the most powerful test [185] is the Rayleigh test [186] which uses the mean resultant length of the projected recoil vectors. With $2 \mathrm{~d}$ axial data the standard procedure is to double the axial angles, reduce them modulo $360^{\circ}$ and analyze the resulting vectorial data [187].

They found that the detector property which has the largest effect on the number of events required to reject isotropy is whether or not the sense of the recoils can be measured. If the senses cannot be measured then the number of events is increased by one order of magnitude for $3 \mathrm{~d}$ read-out and at least two orders of magnitude for $2 \mathrm{~d}$ read-out. For $2 \mathrm{~d}$ read-out this can be reduced to a factor of $\sim 30$ if the reduced angles (with the direction of Solar motion subtracted) are analyzed instead of the raw recoil angles. If the senses are measured then for $2 \mathrm{~d}$ read-out in the optimal plane (which has normal perpendicular the Earth's spin axis) the required exposure is increased by a factor of $\sim 3$ relative to $3 \mathrm{~d}$ read-out. Again this can be reduced, to a factor of $\sim 2$, by using the reduced angles. Note, however, that these numbers assume perfect angular resolution and, due to projection effects, angular resolution will be a more significant factor in $2 \mathrm{~d}$ than in $3 \mathrm{~d}$.

Green and Morgan also investigated the consequences of statistical, rather than event by event, sense determination [48]. If the probability of correctly determining the sense is greater than 0.75 , then the number of events required to reject isotropy using non-parametric statistics is increased by at most a factor of a few. As the probability is decreased below 0.75 then the number of events increases sharply, and eventually it is better to discard the sense information and instead use axial statistics. Note that in the case of a likelihood-based analysis considering also the energy information, not being able to recover the sense of the recoil only impacts the sensitivity of the experiment by about a factor 4 at high WIMP mass [188]. By considering energy dependent sense determination probabilities, they ascertained that correctly determining the sense of the abundant, but less anisotropic, low energy recoils is most important for minimising the number of events required.

With model independent techniques, the background rate effectively places a lower limit on the WIMP cross-section to which the detector is sensitive. It would be very difficult to detect WIMPs using these techniques if the signal rate is more than an order of magnitude below the background rate.

Billard et al. have investigated the effect of experimental limitations on the sensitivity of upcoming directional detection experiments in the context of model-dependent analysis techniques, namely using a profile likelihood test statistics [188]. Considering both the angular and the energy information of the events, they computed the discovery potential for several different experimental configurations (see Fig. 7). To remain robust against halo parameter assumptions, they took into account most of the relevant astrophysical uncertainties, namely halo anisotropy and triaxiality, as nuisance parameters in their analysis. Results from this study considering a $\mathrm{CF}_{4}$ experiment are presented in Fig. 7 where the left panel illustrates the impact of the energy threshold only and the right panel shows the impact of limited angular resolution, sense recognition and background contamination. For convenience, the curves of iso-number of WIMP events are also presented as thin short-dashed lines. From the left panel of Fig. 7 , one can derive that as for direction-insensitive experiments, the energy threshold has a huge impact on the discovery potential and is actually the most important experimental limitation. From the right panel of Fig. 7 , we can first see that a $\mathrm{CF}_{4}$ experiment with perfect sense recognition, $3 \mathrm{~d}$ read-out, a threshold of $5 \mathrm{keV}$ and no background contamination, only 5 WIMP events and 30 WIMP events are required to reach a $3 \sigma$ discovery at WIMP masses of $5 \mathrm{GeV}$ and $1 \mathrm{TeV}$ respectively (black solid line). However, with a background contamination of about 300 events isotropically distributed and flat in energy (red dashed line), these required numbers of WIMP events are increased to 30 and 150 respectively, i.e. a decrease in discovery sensitivity by a factor of 6 . Interestingly, they have shown that as long as the recoil direction and sense are well reconstructed, this result is fairly independent of the assumed energy distribution of the background, highlighting the interest of the directional signature of WIMP events.

Eventually, Billard et al. found that angular resolution and sense recognition efficiency (green and blue dashed lines) could both affect strongly (by about a factor of 4-5) the sensitivity of a directional experiments especially at high WIMP mass (above $100 \mathrm{GeV}$ ). On the other hand, they found that limited energy resolution only affects the experiment's sensitivity in a negligible way, even in extreme and unrealistic cases.

As a conclusion of this study, Billard et al. have shown that the most critical experimental considerations, in order of importance, are the energy threshold, background contamination, sense recognition, angular resolution and energy resolution. Interestingly, they also demonstrated that even a low performance directional detector with a $30 \mathrm{~kg}-\mathrm{year}$ 

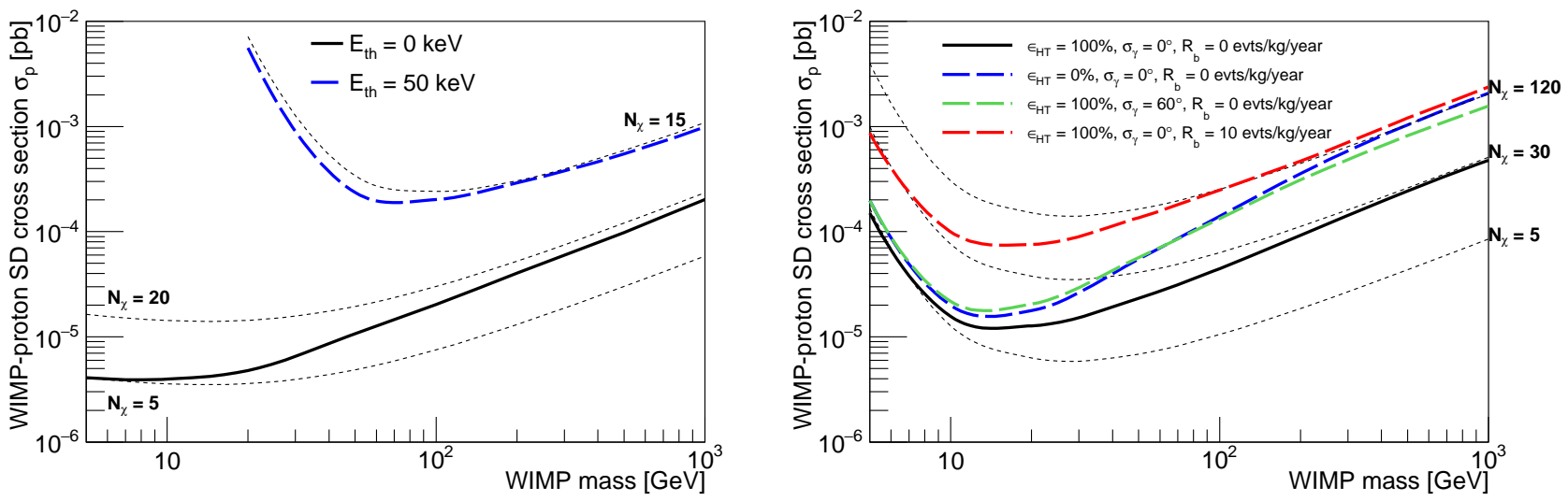

FIG. 7: Lower bound of the $3 \sigma$ discovery region at $90 \%$ C.L. in the $\left(m_{\chi}, \sigma_{p}\right)$ plane with various detector configurations. Left: effect of the energy threshold $\left(E_{t h}\right)$. Black line presents the $E_{t h}=0 \mathrm{keV}$ case, while the dashed blue line presents the same detector with a $E_{t h}=50 \mathrm{keV}$ threshold. Right: effect of the angular resolution $\left(\sigma_{\gamma}\right)$, the sense recognition efficiency $\left(\epsilon_{H T}\right)$ and of the residual background level. Black line presents the background free case with perfect angular resolution $\sigma_{\gamma}=0^{\circ}$ and full sense recognition efficiency $\epsilon_{H T}=100 \%$. Dashed blue line presents the expected performance with a lower angular resolution $\sigma_{\gamma}=60^{\circ}$. Dashed green line presents the expected performance with no sense recognition capability $\left(\epsilon_{H T}=0 \%\right)$. Dashed red line presents the expected performance with a residual background at the level of 10 background events per year per kg. For convenience, the curves of iso-number of WIMP events are presented (dashed lines) with the corresponding number of WIMP events $\left(N_{\chi}\right)$.

exposure, no sense recognition capability, $50^{\circ}$ angular resolution and a background rate of 10 events $/ \mathrm{kg} / \mathrm{year}$, could identify WIMP events for spin-dependent cross section on the proton above $10^{-4} \mathrm{pb}$ [188].

\section{E. Using directionality to overcome the neutrino floor}

Directional detection is particularly interesting for dealing with coherent neutrino-nucleus scattering which represents the ultimate background to direct Dark Matter searches. Neutrinos from the Sun, atmospheric cosmic ray collisions and the diffuse supernova background (DSNB) will become important backgrounds for experiments with sensitivities to WIMP-nucleon spin-independent scattering cross-sections around $10^{-48}-10^{-46} \mathrm{~cm}^{2}[10,72,73]$ and around $10^{-40}-10^{-46} \mathrm{~cm}^{2}$ in the case of spin-dependent interaction [74]. The limiting cross-section at which the neutrino background becomes important is known as the neutrino floor and is dependent on the systematic uncertainty on the flux of various different neutrino contributions for different WIMP mass ranges. For example in a Xenon detector at the low WIMP mass range around $6 \mathrm{GeV}$ the dominant neutrino background is from ${ }^{8} \mathrm{~B}$ Solar neutrinos. At higher WIMP masses between $20-30 \mathrm{GeV}$ the dominant contribution is from the DSNB and around $100 \mathrm{GeV}$ from atmospheric neutrinos. Due to the low fluxes of atmospheric and DSNB neutrinos the floor for WIMP masses higher than $10 \mathrm{GeV}$ appears at much lower cross-sections. Directional detection is a possible way to continue Dark Matter searches below this limit as directional information acts as a discriminator between WIMP and neutrino induced recoils. It should be noted that the neutrino floor is not an absolute limit to direct detection. The floor can in fact be circumvented without directional information in high-statistics analyses using recoil energy alone because of the slight differences in the tails of the recoil energy spectra of WIMPs and neutrinos [74]. Including additional information such as event timing also allows the neutrino and WIMP signals to be distinguished but this too requires very large numbers of events [190].

The triple differential recoil rate per unit detector mass for coherent neutrino-nucleus scattering is the convolution of the double differential cross-section (which is well explained by the standard model, see Ref. [189]) and the neutrino directional flux,

$$
\frac{\mathrm{d}^{3} R}{\mathrm{~d} E_{r} \mathrm{~d} \Omega_{r} \mathrm{~d} t}=\mathscr{N} \int_{E_{\nu}^{\min }} \frac{\mathrm{d}^{2} \sigma}{\mathrm{d} E_{r} \mathrm{~d} \Omega_{r}} \times \frac{\mathrm{d}^{3} \Phi}{\mathrm{d} E_{\nu} \mathrm{d} \Omega_{\nu} \mathrm{d} t} \mathrm{~d} E_{\nu} \mathrm{d} \Omega_{\nu}
$$

where $E_{\nu}^{\min }$ is the minimum neutrino energy required to generate a recoil of energy $E_{r}$ and $\mathscr{N}$ the number of target nuclei. The neutrino directional flux is dependent on the type of neutrino under consideration. For Solar neutrinos 
$0-1.6667 \mathrm{keV}$
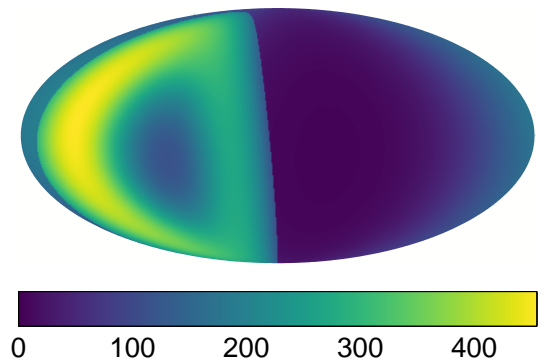

$1.6667-3.3333 \mathrm{keV}$

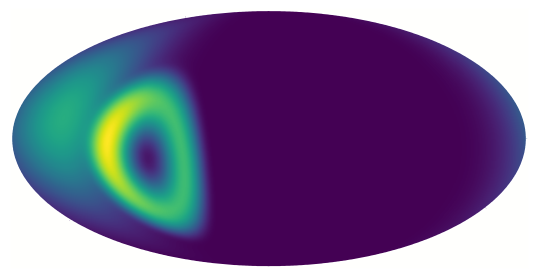

5
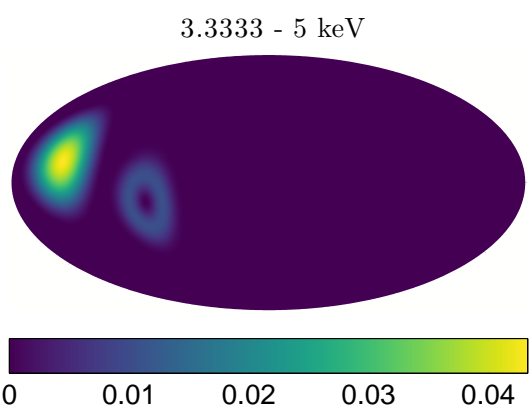

Time: 6th Sep. 2015 06:00
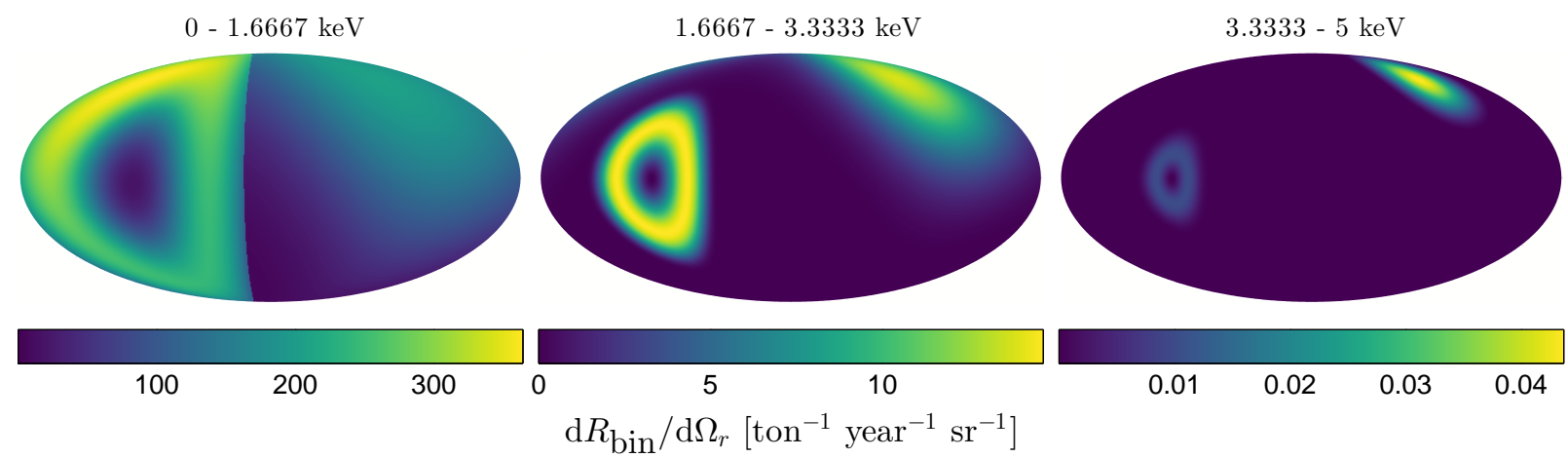

FIG. 8: Mollweide projections of the WIMP plus ${ }^{8} \mathrm{~B}$ neutrino angular differential event rate integrated within (from left to right) three equally sized energy bins spanning the range $E_{r}=0$ to $5 \mathrm{keV}$, for a WIMP with mass $m_{\chi}=6 \mathrm{GeV}$ and $\sigma_{n}^{S I}=4.9 \times 10^{-45} \mathrm{~cm}^{2}$ and a Xe target. The top row shows the signal on February 26th, when the separation between the directions of the Sun and Cygnus is smallest $\left(\sim 60^{\circ}\right)$, and the bottom row on September 6th, when the separation is largest $\left(\sim 120^{\circ}\right)$. The WIMP contribution is to the left of the neutrino contribution on the top row and to the right on the bottom row. The Mollweide projection used in this Figure is of recoil directions in the laboratory co-ordinate system in which the horizontal axis corresponds to a plane parallel to the floor and the top of the map corresponds to the zenith.

the flux is a Dirac delta function

$$
\frac{\mathrm{d}^{3} \Phi}{\mathrm{d} E_{\nu} \mathrm{d} \Omega_{\nu} \mathrm{d} t}=\frac{\mathrm{d} \Phi}{\mathrm{d} E_{\nu}} \frac{1}{\Delta t}\left[1+2 \epsilon \cos \left(\frac{2 \pi\left(t-t_{\nu}\right)}{T_{\nu}}\right)\right] \delta\left(\hat{\mathbf{q}}_{\nu}-\hat{\mathbf{q}}_{\odot}(t)\right)
$$

where $\hat{\mathbf{q}}_{\odot}$ is a unit vector in the direction of the Sun.

The flux has a cosine modulation in time due to the eccentricity of the Earth's orbit, $\epsilon=0.016722$. The time $t_{\nu}=3$ days is the time at which the Earth-Sun distance is shorter and $T_{\nu}=1$ year is the period of modulation. The directional flux for atmospheric and diffuse supernova neutrinos can be approximated as isotropic as the very weak angular dependence becomes washed out in the recoil distribution [189].

The directional signatures of WIMP and neutrino recoils are both unique and can be further discriminated by the correlation of these angular patterns with time. In the sky the position of the Sun follows the path of the ecliptic whereas the direction of motion of the Sun, which corresponds to the constellation Cygnus, remains fixed on the celestial sphere and these two points of origin do not coincide. Figure 8 shows Mollweide projections of the $3 \mathrm{~d}$ angular differential event rate from a $6 \mathrm{GeV}$ WIMP plus ${ }^{8} \mathrm{~B}$ Solar neutrinos at the times when the separation between the directions of the Sun and Cygnus are smallest $\left(60^{\circ}\right)$ and largest $\left(120^{\circ}\right)$ [189]). Even at the time of smallest separation, the WIMP and neutrino recoil distributions can be distinguished as long as the angular resolution is better than a few tens of degrees. Although Fig. 8 only shows the rates for ${ }^{8} \mathrm{~B}$ neutrino induced recoils, the angular distributions for other Solar neutrinos are very similar as neutrinos can only induce a recoil with an angle in the range $(0, \pi / 2)$ from their incident direction. The event rates for atmospheric and DSNB neutrinos are isotropic so are not shown here.

Grothaus et al. first quantitatively explored the impact of direction-sensitivity on the neutrino bound in direct detection [177]. To do this they calculate probability distribution functions for the Dark Matter signal and the 

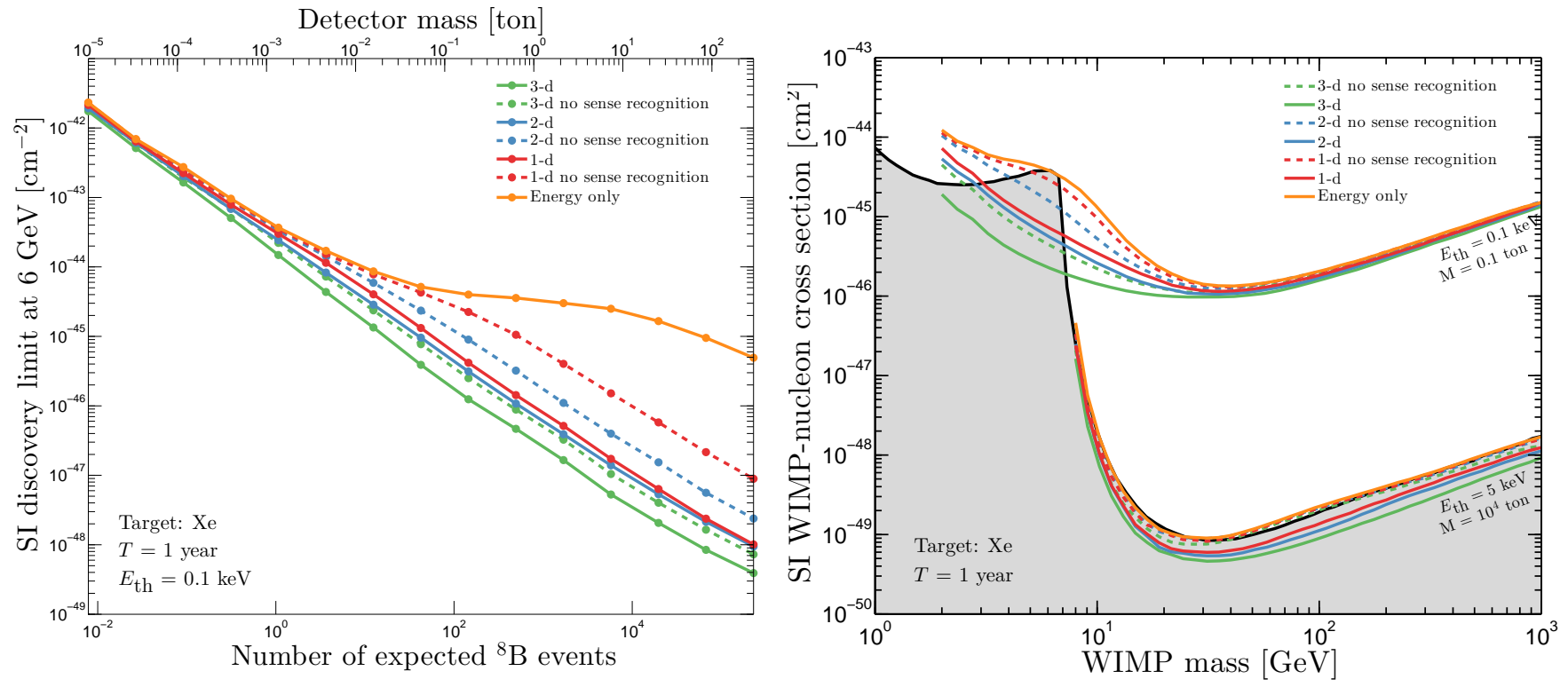

FIG. 9: Left: evolution of the discovery limit for a $6 \mathrm{GeV}$ WIMP as a function of Xenon detector mass. The exposure time was fixed at $T=1$ year and the energy threshold was $0.1 \mathrm{keV}$. The limits shown are for each read-out strategy, $1 \mathrm{~d}$ (red), $2 \mathrm{~d}$ (blue) and 3d (green) in cases both with (solid lines) and without (dashed lines) sense recognition, the limit made by the same detector with no directional information is shown in orange. Right: the discovery limit as a function of WIMP mass for the same read-out strategies as the left panel but with fixed detector set-up. The upper set of limits are for a low threshold-low mass detector $(0.1 \mathrm{keV}, 0.1 \mathrm{ton})$ and the lower set of limits for a high threshold-high mass detector $\left(5 \mathrm{keV}, 10^{4}\right.$ ton). The shaded region shows the neutrino floor from Ref. [10] and the Figures are taken from Ref. [189].

neutrino background, from Solar, atmospheric, and DSNB neutrinos, in the dimensions of recoil energy, recoil direction, and event time. A $\mathrm{CL}_{\mathrm{s}}$ test [191] is performed to distinguish between the neutrino background and background + Dark Matter signal hypotheses. This work considers both $\mathrm{CF}_{4}$ and Xe directional detectors, and includes detector effects by smearing the probability distributions. The detector performance assumptions are moderately optimistic: the angular resolution used is $30^{\circ} / \sqrt{E_{r}}$, the energy threshold is $5 \mathrm{keV}$ in $\mathrm{CF}_{4}$ and $2 \mathrm{keV}$ in $\mathrm{Xe}$, and the nuclear recoil detection efficiency plateaus at $50 \%$. In order to set limits, the $\log$-likelihood ratio $Q=-2 \log \widetilde{Q}$ is used, where $\widetilde{Q}=\mathcal{L}(\vec{X}, S+B) / \mathcal{L}(\vec{X}, B)$ is the ratio of likelihoods of a set of recoils, $\vec{X}$, under signal+background and background only hypotheses respectively [191]. The $90 \%$ confidence level limit is taken to be the cross section value at which the overlap of the background only and signal+background distributions is 0.1 .

The main results from this study are that direction-sensitivity adds approximately an order of magnitude sensitivity beyond non-directional searches for light Dark Matter, and depending on the target species and energy threshold, this sensitivity can leap far beyond the Solar neutrino bound. Further, directionality is more helpful for lighter targets than heavier targets; for the light target material directional information is helpful for the complete Dark Matter mass range, whereas for the heavy target nuclei, directional and non-directional detectors give the same limits for heavy dark matter.

O'Hare et al. followed the work of Ref. $[43,177]$ to study the effect of direction-sensitivity on the neutrino floor for experiments with only $1 \mathrm{~d}$ and $2 \mathrm{~d}$ recoil track information [189]. Figure 9 shows the discovery limits for a Xenon detector located in the Modane underground lab, operated for one year with a range of detector masses. The discovery limits in this work were defined as the minimum cross-section for which $90 \%$ of hypothetical experiments can reach a $3 \sigma$ discovery. They were derived using a profile likelihood ratio test accounting for the systematic uncertainties on the various neutrino fluxes as nuisance parameters. The left panel of Fig. 9 shows the evolution of the discovery limit for a $6 \mathrm{GeV}$ WIMP in a $0.1 \mathrm{keV}$ threshold detector as a function of detector mass. The discovery limits shown are for each read-out strategy: $1 \mathrm{~d}, 2 \mathrm{~d}$, and $3 \mathrm{~d}$ both with and without sense recognition as well as a comparison to a limit obtained by the same detector without any directional information (energy only). The plateauing of the energy-only limit when the signal becomes saturated by neutrino events is what is commonly referred to as the neutrino floor. Including directional information completely removes the neutrino floor at this WIMP mass. In the case of $3 \mathrm{~d}$ read-out with sense recognition, the limits represent the best-case scenario with a scaling going as the inverse of detector mass maintained even to very high neutrino event numbers. In the case of $1 \mathrm{~d}$ read-out without sense recognition which 
is the least powerful directional read-out considered here, cross-sections below the neutrino floor are still accessible, though require higher detector masses.

The right panel of Fig. 9 shows the discovery limit for each read-out strategy now as a function of WIMP mass but for fixed detector mass. Two sets of limits are shown, the upper set correspond to limits obtained by a 0.1 ton detector with a $0.1 \mathrm{keV}$ threshold whereas the lower set of limits are for a $10^{4}$ ton detector with a $5 \mathrm{keV}$ threshold. These masses and thresholds were chosen such that an analogous non-directional experiment would have enough Solar or atmospheric neutrino events for the discovery limit of $\sim 6 \mathrm{GeV}$ and $\sim 100 \mathrm{GeV}$ WIMPs respectively to have entered the saturation regime. This allows the benefit provided by directionality to be fully demonstrated. In these cases the limits show that cross-sections below the neutrino floor can be probed for the full range of WIMP masses though the advantage is only by a factor of a few around $100 \mathrm{GeV}$ where the atmospheric neutrinos are most important. Discriminating the isotropic atmospheric and diffuse supernova neutrino recoils from WIMP recoils is more difficult than with Solar neutrinos as there is more overlap between the angular dependence of the event rates. Also because of this effect the limits obtained without sense recognition around $100 \mathrm{GeV}$ are only marginally better than the energy only limit.

To summarize, direct detection with directional information presents the most powerful approach for disentangling a WIMP signal from the ultimate neutrino background. Directional information is particularly useful for probing light masses as the Solar neutrino events have a very different angular distribution than the WIMP signal. For heavier WIMPs much higher exposures and detector masses are required to distinguish WIMPs from atmospheric and supernovae neutrinos but the floor can still be overcome by a factor of a few if sense recognition is possible. Finally even if only partial directional information is available such as with $1 \mathrm{~d}$ or $2 \mathrm{~d}$ read-outs experiments, the neutrino floor can still be overcome.

\section{OBSERVING SECONDARY FEATURES}

\section{A. Introduction}

In Sections IV C and IV D we discussed two secondary directional features, in addition to the dipole: a ring of maximum recoil rate around the average WIMP arrival direction, and aberration features that are changes in the recoil direction pattern caused by the Earth's revolution around the Sun. In this section we study the prospects of observing the ring and aberration features, and estimate the number of events needed to detect them assuming no background and perfect energy and angular resolutions. We also discuss using aberration features of the directional recoil rate to obtain information on the local WIMP velocity distribution.

\section{B. Observing ring-like features}

As mentioned in Section IV C, it is easiest to observe the ring-like feature when the contrast between the rate at the center of the ring and the ring is largest. The dependence of the ratio $\hat{f}_{\text {center }} / \hat{f}_{\text {ring }}$ on energy can be extracted from Fig. 10. In the left panel of Fig. 10 we show the $\mathrm{F}$ directional differential rate as a function of the polar angle $\theta_{v}$ measured from the average WIMP arrival direction, at different recoil energies for $m_{\chi}=100 \mathrm{GeV}, v_{\mathrm{lab}}=312 \mathrm{~km} \mathrm{~s}$ and $\sigma_{v}=173 \mathrm{~km} \mathrm{~s}^{-1}$ on June 2, assuming WIMPs with SD interactions. The ring is present at all energies below 40 $\mathrm{keV}$.

Since measuring the differential rate will require very large statistics, we study the prospects of observing the ring in the energy-integrated rate. The right panel of Fig. 10 shows the $\mathrm{F}$ directional recoil rate at fixed azimuthal angle, integrated over different energy intervals as a function of the polar angle $\theta_{v}$. To easily see that the ring-like feature exists in the recoil rate integrated over energy intervals from $5 \mathrm{keV}$ up to $40 \mathrm{keV}$ and lower, the energy-integrated rate is re-scaled to be between 0 and 1 . From the right panel of Fig. 10 we can conclude that for low energy thresholds, it is possible to observe a ring in the energy-integrated rate.

The ring-like feature persists in an anisotropic halo, but has different characteristics compared to the isotropic Maxwell-Boltzmann model, which was assumed so far. In the anisotropic logarithmic-ellipsoidal model of Ref. [192], the ring remains circular with constant radius at any azimuthal angle around the average WIMP arrival direction, but the maximum value of the rate depends on the azimuthal angle (see Fig. 14 of Ref. [167]). Thus, some information on the WIMP velocity distribution can be obtained from the ring-like feature, but more work would be necessary to clarify this issue.

The ring-like feature would not exist in the background rate, and hence it can be used as a secondary signature of Dark Matter. If directional detectors can reach low energy thresholds, only around five times more events would be required to detect the ring in the energy-integrated rate, compared with the number required to detect the mean 


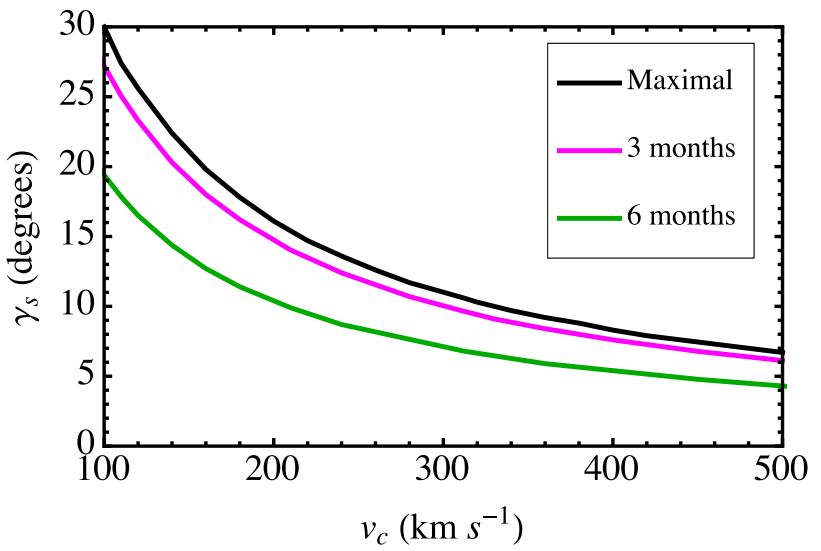

FIG. 11: Maximal angular separation $\gamma_{s}$ between the directions of $-\mathbf{v}_{\text {lab }}$ in a year (black curve), and between the mean incoming recoil directions in two three months periods (magenta curve) and two six months periods (green curve), plotted as a function of $v_{c}$. From Ref. [168].

the three-month-averaged $\gamma_{s}$ would require four times this number of events. If we assume an error of $\Delta \gamma_{s} \simeq 3.5^{\circ}$, the measured $v_{c}$ would be $180_{-30}^{+60} \mathrm{~km} \mathrm{~s}^{-1}$ or $312_{-70}^{+190} \mathrm{~km} \mathrm{~s}^{-1}$ using the three-month-averaged

Therefore, a few thousand events would be needed to measure $\gamma_{s}$ as implied by Refs. [181] and [157]. We discuss next the possibility to detect the annual modulation of the rate integrated over Galactic hemispheres with that same number of events.

The change in $v_{\text {lab }}$ during a year causes the annual modulation of the differential rate [13]. The differential rate is maximum (minimum) at high energies when $v_{\text {lab }}$ is maximum (minimum). At low energies the phase is inverted and the differential rate is maximum when $v_{\text {lab }}$ is minimum. We integrate the directional differential recoil rate over direction to compute the annual modulation amplitude of the energy differential rate,

$$
\Delta\left(\frac{\mathrm{d} R}{\mathrm{~d} E_{r}}\right)=\frac{\mathrm{d} R_{\max }}{\mathrm{d} E_{r}}-\frac{\mathrm{d} R_{\min }}{\mathrm{d} E_{r}}=\int\left(\frac{\mathrm{d}^{2} R_{\max }}{\mathrm{d} E_{r} \mathrm{~d} \Omega_{r}}-\frac{\mathrm{d}^{2} R_{\min }}{\mathrm{d} E_{r} \mathrm{~d} \Omega_{r}}\right) \mathrm{d} \Omega_{r},
$$

where the subscripts "max" and "min" refer to the maximum and minimum differential rates during a year.

Alternatively, we can integrate the directional differential rate over the recoil directions pointing to half of the sky and define the Galactic Hemisphere Annual Modulation (GHAM) [168]. For specific hemispheres, the GHAM amplitude is larger than the usual annual modulation amplitude, and therefore easier to detect. The Galactic hemispheres which are divided by planes perpendicular to the Earth's orbit around the Sun have the largest GHAM, since the total orbital velocity is in the direction of one hemisphere at some time and away from it half a year later. The two hemispheres with the maximum GHAM are complimentary: in one the rate is maximum when $v_{\text {lab }}$ is maximum, while in the other the rate is maximum when $v_{\text {lab }}$ is minimum.

We next study the GHAM in the energy-integrated rate. The annual modulation amplitude of the energy-integrated rate is $\Delta R=R_{\max }-R_{\min }$, where $R_{\max }$ and $R_{\min }$ are the maximum and minimum energy-integrated rates during a year integrated over an energy interval $\left[E_{1}, E_{2}\right]$. The annual modulation amplitudes of $\mathrm{F}$ recoil rates integrated above $E_{r}$, as a function of $E_{r}$, for the two hemispheres with the maximum GHAM amplitude at high (magenta curve) and low (green curve) energies, as well as over the total sky (black curve) are plotted in Fig. 12, for a $100 \mathrm{GeV}$ WIMP with SD interactions and four different combinations of $v_{c}$ and $\sigma_{v}$. We show the difference between the rates at dates when $v_{\text {lab }}$ is maximum and minimum. Therefore, the amplitudes at high and low energies $\left(E_{r}\right.$ greater/less than $\sim 10$ to $20 \mathrm{keV}$ depending on the value of $v_{c}$ and $\sigma_{v}$ ) are shown as positive and negative, respectively, while the sum of the two is the usual non-directional annual modulation. At low energies, the magenta GHAM amplitude is two to three times larger than the non-directional modulation amplitude. One can see from the two panels of Fig. 12 that the magnitude and shape of the GHAM amplitudes as a function of recoil energy strongly depend on $v_{\text {lab }}$ and $\sigma_{v}$. The annual modulation amplitude which is approximately the annual average rate times $\left(v_{\mathrm{e}, \mathrm{rev}} / v_{\text {lab }}\right)$, is larger for smaller $v_{\text {lab }}$. Furthermore, for smaller $\sigma_{v}$ the recoil rate is more anisotropic, and thus the rate difference is larger. Hence, one obtains the largest GHAM amplitudes of the energy differential rates for the smallest $v_{\text {lab }}$ and $\sigma_{v}$. However, more work is needed to quantify the dependence of the GHAM amplitudes on $v_{\text {lab }}$ and $\sigma_{v}$ in the energy-integrated rate.

To observe the annual modulation at the $3 \sigma$ level, the difference between the number of events during the annual half-cycles with high and low rates must be greater than $3 \sqrt{N_{\text {tot }} / 2}$, where $N_{\text {tot }}$ is the number of events in both cycles. Using this statistical test, one can estimate the minimum number of events necessary to detect the GHAM [168]. The 

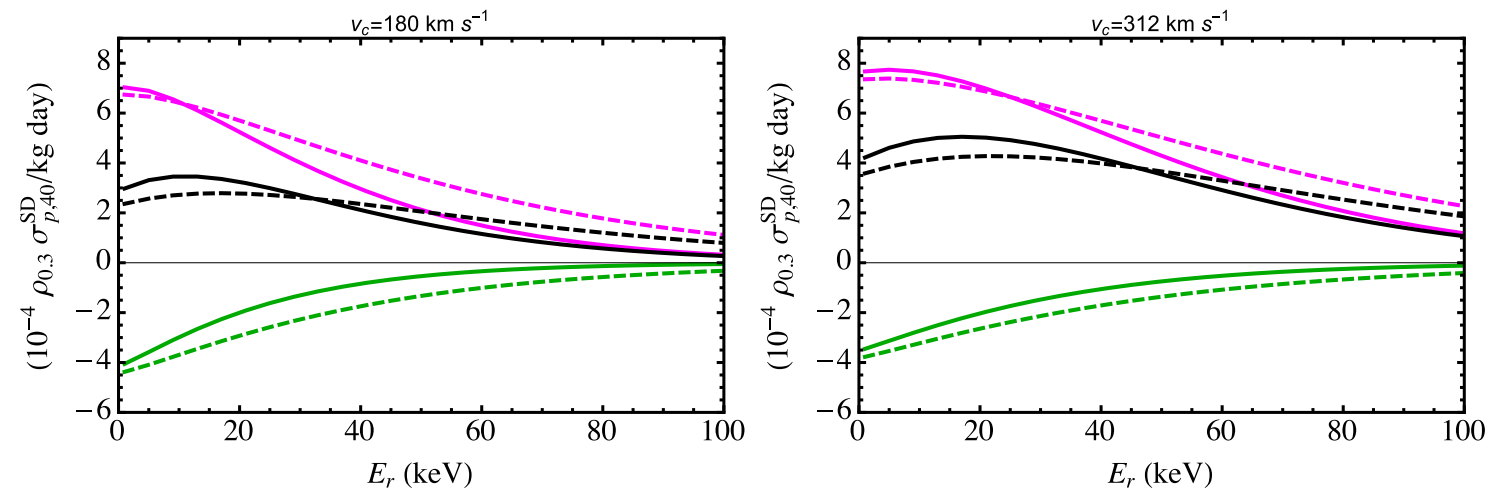

FIG. 12: Annual modulation amplitude of the energy-integrated recoil rate in a Fluorine detector as a function of the recoil energy $E_{r}$ and integrated above $E_{r}$ and over the two hemispheres with the largest GHAM amplitude at high (magenta curves) and low (green curves) energies, and over the total sky (black curves). In the left and right panels we assume $v_{c}=180 \mathrm{~km} \mathrm{~s}^{-1}$ and $312 \mathrm{~km} \mathrm{~s}^{-1}$, respectively. The solid and dashed curves correspond to $\sigma_{v}=173 \mathrm{~km} \mathrm{~s}^{-1}$ and $225 \mathrm{~km} \mathrm{~s}{ }^{-1}$, respectively. In both panels we assume $m_{\chi}=100 \mathrm{GeV}, v_{\text {esc }}=544 \mathrm{~km} \mathrm{~s}^{-1}$, and SD interactions.
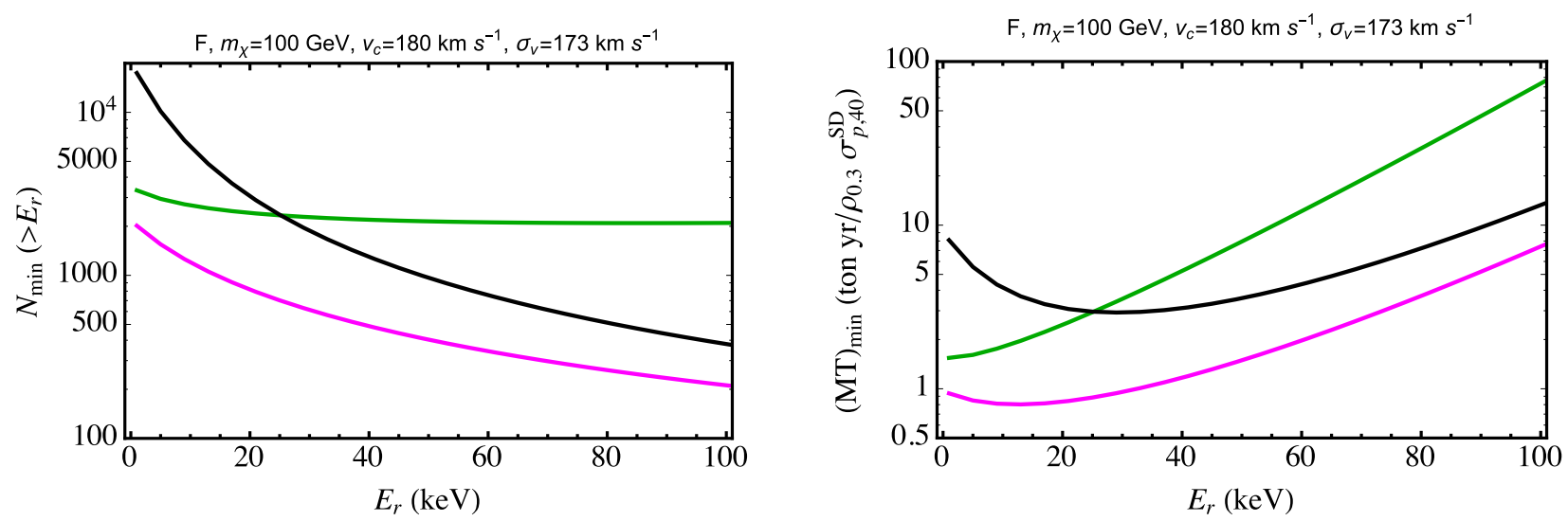

FIG. 13: Minimum number of events (in all directions) with energy larger than $E_{r}$ (left panel), and the exposure $M T$ (in tonyr) corresponding to the minimum number of events (right panel) needed to detect (at the $3 \sigma$ level) the usual non-directional annual modulation (black curve) and the largest GHAM amplitude at low (green curve) and high (magenta curve) energies in an $\mathrm{F}$ detector, assuming SD interactions. The parameters used are $m_{\chi}=100 \mathrm{GeV}, v_{\mathrm{esc}}=544 \mathrm{~km} \mathrm{~s}^{-1}, v_{c}=180 \mathrm{~km} \mathrm{~s}{ }^{-1}$ and $\sigma_{v}=173 \mathrm{~km} \mathrm{~s}^{-1}$.

left panel of Fig. 13 shows the minimum number of events with energy larger than $E_{r}$ (in all directions), needed to detect the total annual modulation (black curve), and the maximum GHAM amplitude at low (green curve) and high (magenta curve) energies at the $3 \sigma$ level in a Fluorine detector. We assume $m_{\chi}=100 \mathrm{GeV}$ with SD interactions, and the combination of low $v_{c}$ and $\sigma_{v}, 180 \mathrm{~km} \mathrm{~s}^{-1}$ and $173 \mathrm{~km} \mathrm{~s}^{-1}$, respectively, which gives the smallest minimum number of events. A minimum of a few thousand events are needed to observe the largest GHAM amplitudes, and at least 10 times more is required to observe the usual non-directional annual modulation. The right panel of Fig. 13 shows the minimum exposure needed to detect the non-directional annual modulation (black curve), and the maximum GHAM amplitude at low (green curve) and high (magenta curve) energies in the rate integrated above $E_{r}$.

The minimum number of events with energy above $E_{r}$ in all directions and the minimum exposure needed to detect the largest GHAM amplitude at the $3 \sigma$ level at high energies assuming $m_{\chi}=50 \mathrm{GeV}$ with $\mathrm{SD}$ interactions in a $\mathrm{CF}_{4}$ detector are plotted in the left and right panels of Fig. 14. The four curves in Fig. 14 correspond to four different combinations of $v_{c}$ and $\sigma_{v}$. One can see from Fig. 14 that between a few hundred and a few thousand events are needed to observe the largest GHAM amplitude, and the minimum exposure required to detect the largest GHAM assuming $\sigma_{p}^{\mathrm{SD}}=10^{-40} \mathrm{~cm}^{2}$ is between 0.6 ton-yr and a few tens of ton-yr, depending on the value of $v_{c}$ and $\sigma_{v}$ and the energy interval.

In summary, aberration features can provide information on the WIMP velocity distribution, as well as being a way to confirm the Galactic origin of WIMPs. We would need extremely large exposures to observe the full aberration 

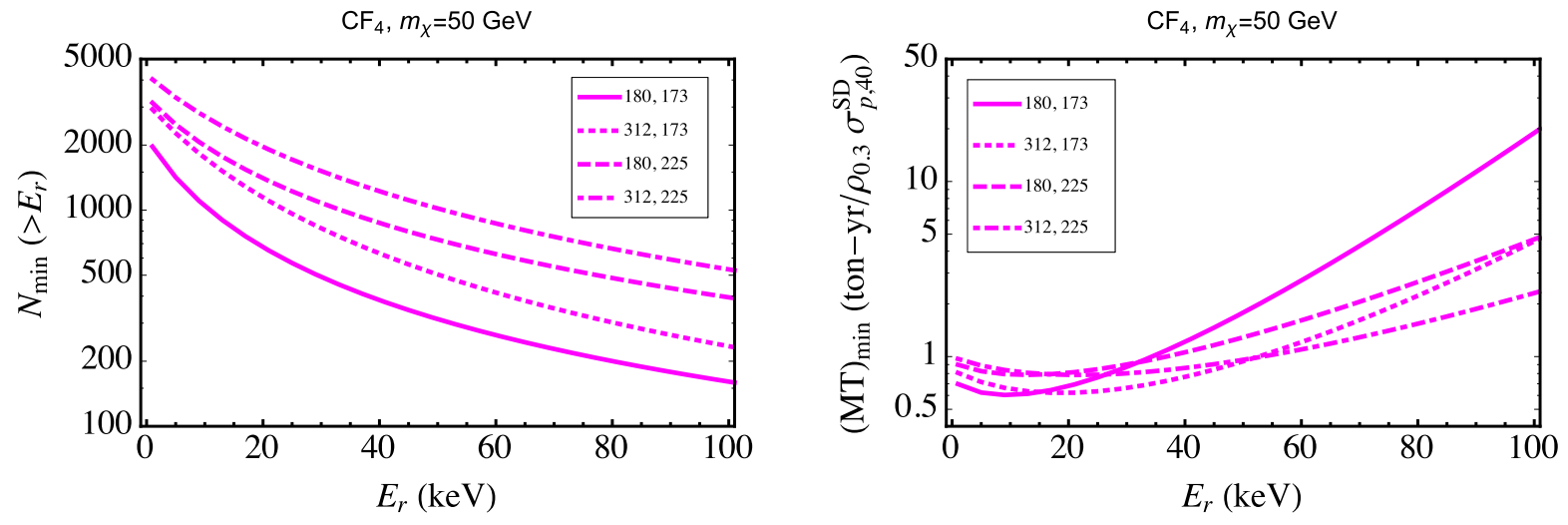

FIG. 14: Minimum number of events in all directions (left panel), and minimum exposure $M T$ (right panel) needed to detect (at the $3 \sigma$ level) the largest GHAM amplitude at high energies in the SD rate integrated above $E_{r}$ in $\mathrm{CF}_{4}$. Different curves correspond to different combinations of $v_{c}$ and $\sigma_{v}$, as indicated by the first and second numbers in the plot legends, respectively. From Ref. [168].

pattern, but observing the annual change in the mean incoming recoil direction or the number of events over specific solid angles may be possible with moderately large exposures. Moreover, given the energy spectrum of the events over specific solid angles, one can obtain some information on $v_{\text {lab }}$ and $\sigma_{v}$, since the magnitude and shape of the GHAM amplitudes as a function of recoil energy strongly depend on $v_{\text {lab }}$ and $\sigma_{v}$, as well as any anisotropy in the DM velocity distribution. More work is needed to determine the best way of extracting the characteristics of the local WIMP velocity distribution using aberration features.

\section{DARK MATTER IDENTIFICATION}

\section{A. Introduction}

Constraining the properties of Dark Matter is the main goal of upcoming detectors, once conclusive evidence in favor of a discovery is observed by at least one experiment. It concerns the properties of both the WIMP particle (mass and cross section) and the Galactic Dark Matter halo (three dimensional local WIMP velocity distribution and density). This topic may also be dealt with via either indirect detection [193] and direct detection separately [137, 193-199], or within a combined analysis [200,201] or in combination with collider data [202] or with the measurements of halo star kinematics [203]. However, directional detection offers a unique opportunity to constrain Dark Matter properties (particle and halo) with the results of a single experiment, thanks to the measurement of the double-differential spectrum $\mathrm{d}^{2} R / \mathrm{d} E_{r} \mathrm{~d} \Omega_{r}$. The idea [157] is to constrain the WIMP properties with the help of a high dimensional multivariate analysis and within the framework of a general halo model, i.e. with a large number of parameters. Several studies have been carried out on the subject $[71,157,158]$ and the free parameters considered may be:

- $m_{\chi}$, the WIMP mass,

- $\sigma_{N}$, the WIMP-nucleon cross section (assumed either SI or SD),

- $v_{\text {lab }}$, the lab velocity with respect to the halo,

- $v_{\text {esc }}$, the escape speed,

- $\sigma_{v}$, the WIMP velocity dispersion of the standard halo model,

- $\left(\sigma_{x}, \sigma_{y}, \sigma_{z}\right)$ the three velocity dispersions in the case of a triaxial halo,

- $\left(\ell_{\odot}, b_{\odot}\right)$, the main incoming direction of the recoiling nuclei,

- $R_{b}$, the background event rate in a given energy range. 


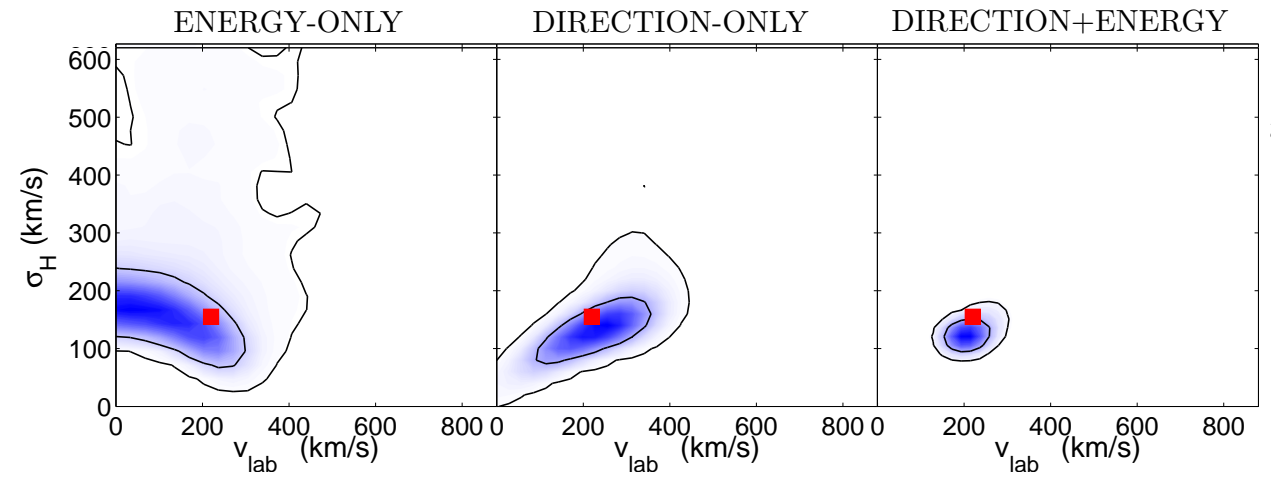

FIG. 15: Posterior probability distribution (68\% and 95\% CL contours) for the WIMP-wind speed $v_{\text {lab }}$ and velocity dispersion $\sigma_{v}$ parameters of a Maxwellian velocity distribution, for a data set consisting of 100 simulated events and assuming $m_{\chi}=50 \mathrm{GeV}$ and a MIMAC-like experiment $\left(\mathrm{CF}_{4}\right.$ with an energy range 5-50 keV, ). Red square markers indicate the fiducial parameter values assumed in simulating the data. Analyses using energy-only, direction-only, and direction+energy information on the distribution of recoil events are shown from left to right respectively. Fig. from Ref. [158].

The utimate goal of directional detection is to extract the posterior PDF of all parameters with the results of a single experiment. However, depending on the assumptions or on the goal of the study, a subset of parameters may be considered as fixed. In the following, we first present in Sec. VIII B a study that highlights the power of directional detectors over experiments with sensitivity only to the energy spectrum [158]. Then, Sec. VIII C demonstrates that a single directional detection experiment may be used to constrain both the WIMP and halo parameters within the framework of a dedicated Markov Chain Monte Carlo analysis (MCMC) [157].

\section{B. Comparing directional and direction-insensitive detection}

In order to compare direction-sensitive and direction-insensitive detection, we first present a study of their ability to constrain the WIMP velocity distribution [158]. To do so, the WIMP mass is assumed to be known, in order to study the effect on the velocity distribution only. The standard halo model (SHM) is considered, given by a Maxwellian velocity distribution and characterized by: the WIMP velocity dispersion $\sigma_{v}$, the relative velocity of the lab with respect to the halo $v_{\text {lab }}$, and the escape velocity $v_{\text {esc }}$. The simulated dataset is: 100 recoil events, for a 50 GeV WIMP, observed with a zero-background experiment. All parameters are supposed to be known exactly, except $v_{\text {lab }}$ and $\sigma_{v}$, which are to be constrained by data.

Figure 15 presents the $68 \%$ and $95 \%$ CL contours obtained with a mock experiment measuring the energy only (left), the direction only (middle) and the double-differential spectrum $d^{2} R /\left(d E_{r} d \Omega_{r}\right)$ (right). The complementarity of the energy-only and direction-only information is easily understood by considering how the energy spectrum and directional distribution of events depend on the parameters $v_{\text {lab }}$ and $\sigma_{v}$. For example, smaller values of $v_{\text {lab }}$ result in a softer energy spectrum, as do smaller values of $\sigma_{v}$. In contrast, smaller values of $v_{\text {lab }}$ yield a more isotropic directional distribution, as do larger values of $\sigma_{v}$. Thus, the direction-only and energy-only measurements provide orthogonal sets of information on these parameters. A straightforward conclusion is that directional detection provides an uncorrelated estimation of these two parameters, as can be seen on Fig. 15. Such parameter-estimation studies nicely demonstrate the power of directional detectors over experiments with sensitivity only to the energy spectrum, as illustrated in Fig. 15.

\section{Constraining halo and WIMP properties}

In this section, we study how directional detection can overcome the WIMP parameter degeneracies, from both the halo and particle physics models considering a benchmark input model described in Ref. [157]: a standard Dark Matter halo (multivariate Gaussian isothermal sphere) composed of $50 \mathrm{GeV}$ WIMPs with a WIMP-nucleon SD cross section $\sigma_{N}=10^{-3} \mathrm{pb}$. Data are simulated for a $10 \mathrm{~kg} \mathrm{CF} 4$ detector with a three-year-exposure time and a background rate of 10 events $/ \mathrm{kg} /$ year described by a flat energy spectrum and an isotropic angular distribution. Perfect energy and angular resolutions are considered. Tab. II presents the constraints on the free parameters obtained from the analysis of the simulated events. Several conclusions may be drawn:

- the free parameters are strongly constrained with no bias with respect to their input values, 
- the proof of discovery is given by the reconstruction of the main incoming direction $\left(\ell_{\odot}, b_{\odot}\right)$, as in Ref. [71], which corresponds to the direction of the Sun's velocity vector within 2.5 degree.

- constraints are obtained on both the Galactic halo, via the velocity dispersions (Sec. VIII C 1) as well as on the WIMP itself $\left(m_{\chi}, \sigma_{N}\right)$, see Sec. VIII C 2 .

Note that the background rate is also a free parameter that is also constrained in the analysis, mainly by the angular part of the spectrum, in the hemisphere opposite to the constellation Cygnus where the expected number of WIMP events is very small. The parameter estimation is thus not affected by the residual background [157]. We now explore in more detail how well the halo and particle physics properties of Dark Matter are constrained.

\begin{tabular}{|c||c|c|c|c|c|c|c|c|c|}
\hline & $m_{\chi}(\mathrm{GeV})$ & $\log _{10}\left(\sigma_{N}(\mathrm{pb})\right)$ & $\ell_{\odot}\left(^{\circ}\right)$ & $b_{\odot}\left({ }^{\circ}\right)$ & $\sigma_{x}\left(\mathrm{~km} \cdot \mathrm{s}^{-1}\right)$ & $\sigma_{y}\left(\mathrm{~km} \cdot \mathrm{s}^{-1}\right)$ & $\sigma_{z}\left(\mathrm{~km} \cdot \mathrm{s}^{-1}\right)$ & $\beta$ & $R_{b}\left(\mathrm{~kg}^{-1} \mathrm{year}^{-1}\right)$ \\
\hline \hline Input & 50 & -3 & 90 & 0 & 155 & 155 & 155 & 0 & 10 \\
\hline Output & $51.8_{-19.4}^{+5.6}$ & $-3.01_{-0.08}^{+0.05}$ & $92.2_{-2.5}^{+2.5}$ & $2.0_{-2.5}^{+2.5}$ & $158_{-17}^{+15}$ & $164_{-26}^{+27}$ & $145_{-17}^{+14}$ & $-0.073_{-0.18}^{+0.29}$ & $10.97 \pm 1.2$ \\
\hline
\end{tabular}

TABLE II: Comparison between the input values and the values obtained with the MCMC analysis, i.e. mean values and 68 \% CL uncertainties. Table from Ref. [157].

\section{Constraining Dark Matter halo properties}

In order to constrain the properties of the Dark Matter halo, a multivariate Gaussian WIMP velocity [192] is considered. It corresponds to the simplest triaxial generalization of the standard isothermal sphere with a density profile $\rho(r) \propto 1 / r^{2}$. In this case, the three velocity dispersions $\left(\sigma_{x}, \sigma_{y}, \sigma_{z}\right)$ are the parameters to be constrained. However, they are slightly correlated with each other, with the WIMP properties $\left(m_{\chi}, \sigma_{N}\right)$ and with the background rate $R_{b}$, see Fig. 3 in [157]. For instance, increasing the velocity dispersions leads to an increase in the number of expected WIMP events and to a wider WIMP event angular distribution. The latter can be compensated by decreasing the WIMP mass as it leads to a tighter angular distribution $[71,176]$. The posterior PDF of the $\beta$ parameter is computed from Eq. 4 and corrected [157] to account for the fact that flat prior for the three velocity dispersions does not correspond to a flat prior for $\beta$.

Mock data have been simulated for an isotropic $(\beta=0)$ Dark Matter halo (tab. II). Using directional detection, it is possible to evaluate the value of $\beta$. Billard et al. [157] found in this case: $\beta=-0.073_{-0.18}^{+0.29}$ (68\% CL), which is in good agreement with isotropy. As shown on figure 16 (left), this result holds true when varying the input WIMP mass, as the constraint on $\beta$ remains consistent with the input value. However, a larger input WIMP mass leads to larger error bars. In addition, even for an extremely triaxial halo model $(\beta=0.4)$, directional detection would still enable a reconstruction of $\beta$. In that case the constraint is $\beta=0.38_{-0.1}^{+0.2}$. In fact, the constraint gets even stronger with increasing input value of $\beta$, see Fig. 16 (right), in agreement with the expected decrease of the degeneracy between the 3 velocity dispersions with increasing departure from isotropy.

In conclusion, data from directional detection may be used to constrain the WIMP velocity dispersions, and hence the properties of the Dark Matter halo, without any assumption on the properties of the Dark Matter particle, as its mass and cross section are considered as free parameters that are also constrained by the analysis, as discussed in the next subsection.

\section{Constraining WIMP properties}

The evaluation of the WIMP mass and cross section gives information on the properties of the Dark Matter particle that in turn may be used to constrain the theoretical particle model (see Sec. IX). As shown in Tab. II, directional detection would enable to constrain the WIMP parameters, with a small dispersion and no bias. The combined use of angular and energy information allows us to obviate bias in the determination of the WIMP properties as degeneracies amongst the free parameters are removed. We refer the reader to Ref. [137] for a detailed discussion about the effect of halo model uncertainties on allowed regions.

Figure 17 (left) presents the constraint on the mass and cross section parameters. A strong correlation is observed and explained by the fact that the event rate scales with $\sigma_{N} / m_{\chi}$ for low mass target nuclei. It can be seen that directional detection would enable us to contrain the WIMP mass and cross section, for any input WIMP mass [71]. However, the constraints depend strongly on the input value of the WIMP mass. Indeed, the constraints on 

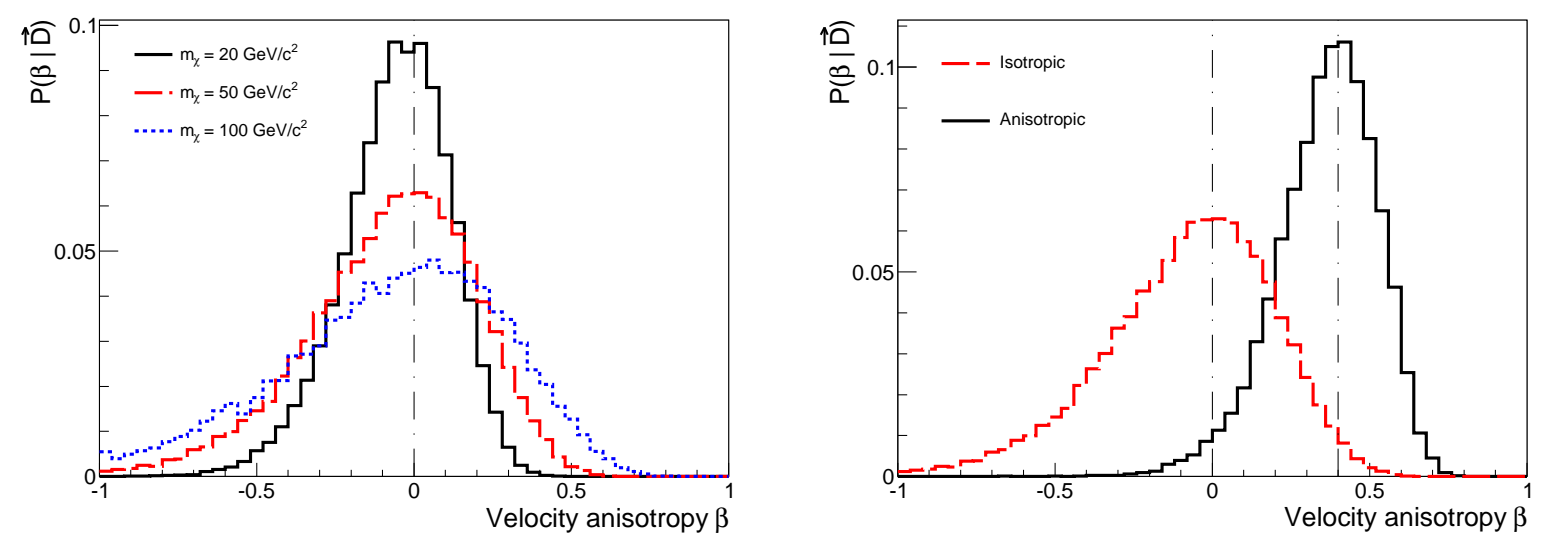

FIG. 16: Left: posterior PDF distribution of the $\beta$ parameter for the isotropic input halo model $(\beta=0)$ and for a WIMP mass equal to 20,50 and $100 \mathrm{GeV}$. Right: posterior PDF distribution of the $\beta$ parameter, for a $50 \mathrm{GeV}$ WIMP and for two input halo models : isotropic $(\beta=0)$ and triaxial $(\beta=0.4)$. Fig. from [157].
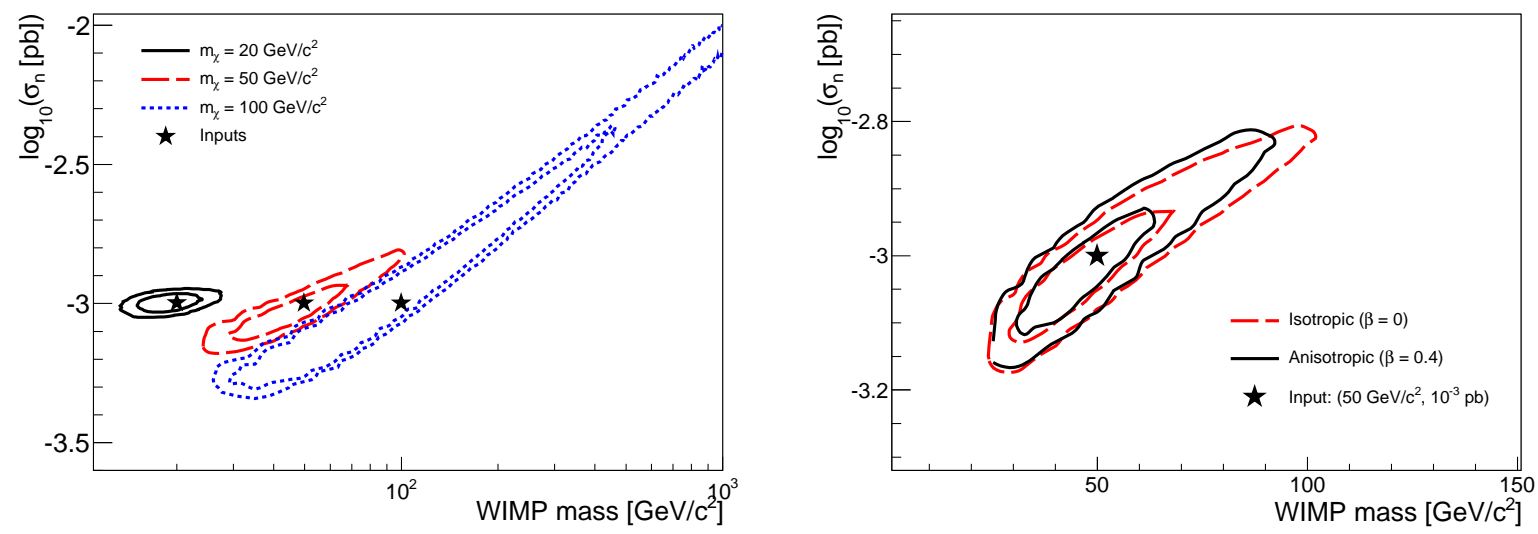

FIG. 17: Left: $68 \%$ and $95 \%$ contour level in the $\left(m_{\chi}, \sigma_{N}\right)$ plane, for the isotropic input model and for a WIMP mass equal to 20, 50 and $100 \mathrm{GeV}$. Right: $68 \%$ and $95 \%$ contour level in the $\left(m_{\chi}, \sigma_{N}\right)$ plane, for a $50 \mathrm{GeV}$ WIMP and for two input models: isotropic $(\beta=0)$ and triaxial $(\beta=0.4)$. Fig. from [157].

$\left(m_{\chi}, \log _{10}\left(\sigma_{N}\right)\right)$ become wider for increasing WIMP mass and only a lower limit may be deduced for heavy WIMPs $\left(m_{\chi} \geq 100 \mathrm{GeV}\right)$, in the case studied i.e. a fluorine target and a recoil energy in the range $[5,50] \mathrm{keV}$.

As shown in Fig. 17 (right), the two halo models $(\beta=0$ and $\beta=0.4)$ give similar constraints that are consistent with the input values. This shows that the bias induced by an incorrect halo model assumption is avoided thanks to the fact that the halo model may also be constrained when analysing directional data. This is a key advantage of directional detection compared to direction-insensitive detectors [137].

In conclusion, directional detection provides a unique opportunity to constrain, with a single experiment, the WIMP mass and the WIMP-nucleon cross section within the framework of a high dimensional multivariate analysis. No $a$ priori assumptions on the isotropy of the halo is needed. This is of great interest in the context of phenomenological efforts $[137,193-197,202,204-206]$ trying to constrain the WIMP parameters $\left(m_{\chi}, \sigma_{N}\right)$ with upcoming Dark Matter experiments. It is of course possible to include external data as nuisance parameters, e.g. measurement of the local Dark Matter density $\rho_{0}[156,207]$ or the escape velocity (taken as infinity in this study). 


\section{PROBING THE INTERACTION OF DARK MATTER}

\section{A. Introduction}

For the standard SD and SI interaction, directionality brings potentially improved sensitivity as it is more robust against backgrounds than direction-insensitive experiments. In particular, measuring the mean recoil direction has been proven to be a powerful tool to reach a $3 \sigma$ discovery of Dark Matter, even with a low number of WIMP events and a high number of background events. As shown in Ref. [155], a $30 \mathrm{~kg}$-year $\mathrm{CF}_{4}$ directional detector would enable a $3 \sigma$ discovery for supersymmetric WIMPs below $m_{\chi} \leq 200 \mathrm{GeV}$. However, the constraints on model parameters, derived from directional or non-directional experiments facing irreducible backgrounds, are of similar nature. Regardless of directional-sensitivity, if an experiment enables a measurement of the WIMP mass and the WIMP-nucleon cross section (see Sec. VIII), conclusions may be drawn on the underlying particle physics model (e.g. supersymmetry, see for instance Ref. [155]). Note also that the complementarity with other Dark Matter search strategies is not an issue specific to directional detection. Indeed, it relies on the choice of the target nucleus, sensitive to either SD, SI interactions or both. Hence, all previous comparisons between direct, indirect and colliders searches for Dark Matter apply, e.g. Refs. [5, 74, 147, 150, 208, 209].

In this section, we discuss how directional detectors may have additional benefits if one considers Dark Matter models with non-standard WIMP nucleon scattering. The case of inelastic Dark Matter is presented in Sec. IX B. In Sec. IX C we present a general framework (see Sec. IIIF), which includes all non-relativistic (NR) operators describing the WIMP-nucleon interaction. We focus on the importance of directional detection in distinguishing between different operators and thus probing the interactions of Dark Matter. Note that directional detection has also been proposed as a tool to study and constrain stable bound state of two asymmetric dark matter particles (darkonium) [210].

\section{B. Using directionality to constrain inelastic Dark Matter}

Inelastic Dark Matter (iDM) [211] is interesting as an example of how directional detectors can have enhanced sensitivity to exotic Dark Matter scenarios that go beyond the standard assumptions of elastic WIMP-nucleon scattering. In the originally proposed iDM scenario, the WIMP has two states whose masses differ by $\delta$, and interacts with nuclei by transitioning from the lighter to the heavier state. As a result, only WIMPs with sufficient kinetic energy to create the heavier state can scatter. This corresponds to the requirement

$$
\delta<\frac{v^{2} m_{\chi} m_{A}}{2\left(m_{\chi}+m_{A}\right)}
$$

where $v$ is the WIMP velocity in the lab frame, $m_{\chi}$ the mass of the lighter WIMP state, and $m_{A}$ the mass of the target nucleus. This means that slow WIMPs cannot scatter. Furthermore, the minimum velocity required for scattering is higher for lighter target nuclei, so that experiments with light target nuclei are at a particular disadvantage. When the original iDM model was proposed, a mass difference of order $\delta=100 \mathrm{keV}$ thus made it possible to reconcile the putative signal seen by DAMA/LIBRA with otherwise conflicting limits from CDMS [212], as the latter experiment has a lighter target nucleus. Although this particular iDM model has since been ruled out [213], the general concept of inelastic Dark Matter remains viable.

Of particular interest for directional detection, the velocity threshold in iDM models means that more recoils occur near threshold, and therefore are more correlated with the direction of incoming WIMPs, which can enhance the signal discrimination power of a directional detector. This was studied by Finkbeiner et al. [214] and Lisanti and Wacker [215]. Finkbeiner et al. [214] concluded that a directional detector with a heavy target gas, such as Xenon, would have been able to probe iDM models (that were viable at the time the paper appeared) with a surprisingly modest exposure. For a $50 \mathrm{keVr}$ detector energy threshold, an exposure of $1000 \mathrm{~kg}$-days was sufficient to exclude or support iDM benchmark models consistent with DAMA. In the case of iDM models, this exposure also would have allowed a significant measurement of the parameter $\delta$ and provided an order-of-magnitude measurement of $m_{\chi} / \sigma_{n}$, the ratio of the WIMP mass to the WIMP-nucleon cross section. Following up on this work, Lisanti and Wacker [215] have shown how, in models with both elastic and inelastic contributions to scattering, directional detectors can set limits on or separate the two components, and hence shed light on the underlying dynamics of more involved Dark Matter models. 


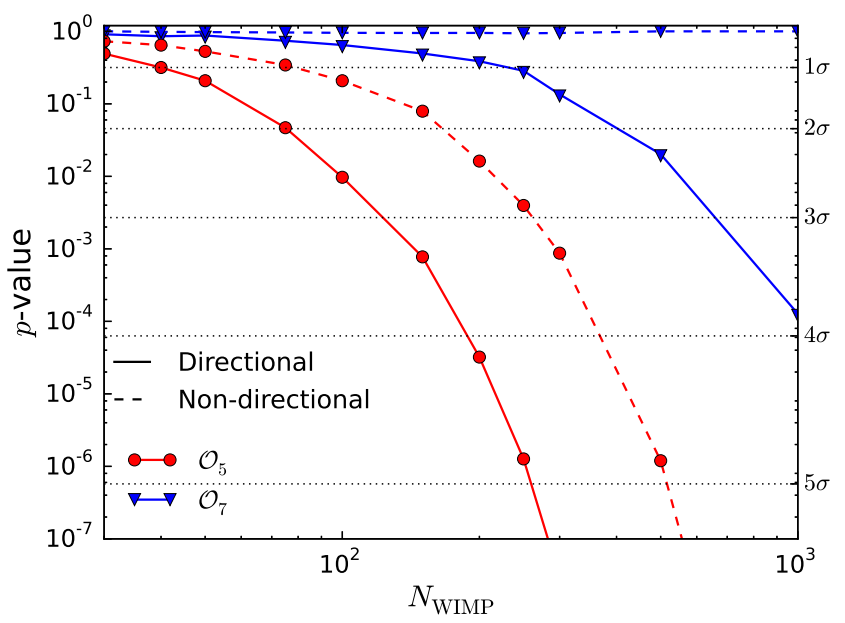

FIG. 18: The $p$-value obtained in $95 \%$ of experiments as a function of the number of signal events $N_{\text {WIMP }}$ for the null hypothesis of standard SD-only interactions. We also show the corresponding confidence level with which standard interactions can be rejected (in units of $\sigma$ ). The signal is distributed according to the non-relativistic operators $\mathcal{O}_{5}$ (red circles) or $\mathcal{O}_{7}$ (blue triangles) [162], with an input WIMP mass of $50 \mathrm{GeV}$. A Fluorine-based detector with an energy threshold of 20 keV is assumed. Experiments with and without directional information are shown as solid and dashed lines respectively. Note that the curve for $\mathcal{O}_{7}$ using only non-directional information lies above $p>0.9$ for all values of $N_{\text {WIMP }}$ considered.

\section{Distinguishing non-relativistic operators}

Directional sensitivity also provides a tool for distinguishing between different interactions within the framework of non-relativistic Dark Matter-nucleon operators (described in Sec. III F). For mock events in a directional detector, generated assuming a particular non-relativistic operator, Ref. [165] examined how many events would be required to reject standard SD-only interactions at a given significance. This was achieved using a likelihood ratio test with 10000 mock datasets for each operator. In each case, the mock data were used to fit the WIMP mass $m_{\chi}$ and $A$, the ratio of standard (SD) to non-standard (non-relativistic) interactions.

The results for an idealised $\mathrm{CF}_{4}$ detector with $20 \mathrm{keV}$ energy threshold and perfect angular resolution are shown in Fig. 18. Dashed lines show the discrimination significance achieved in $95 \%$ of experiments when only event energies are known, while solid lines show the results when directional information is included. The two operators considered are

$$
\mathcal{O}_{5}=i \mathbf{S}_{\chi} \cdot\left(\frac{\mathbf{q}}{m_{N}} \times \mathbf{v}^{\perp}\right) \text { and } \mathcal{O}_{7}=\mathbf{S}_{N} \cdot \mathbf{v}^{\perp}
$$

The first of these operators couples to the recoil momentum as well as the transverse velocity, while the second couples only to the transverse velocity. Using only energy information, it is not possible to reject standard SD interactions if the underlying interaction is due to $\mathcal{O}_{7}$, even with 1000 events. This is because, assuming a SHM velocity distribution, the energy spectrum for SD interactions is indistinguishable from that of $\mathcal{O}_{7}$ (or any NR operator for which the cross section is proportional to $v_{\perp}^{2}$ ). However, including directional information (solid blue line) allows standard interactions to be rejected; roughly 400 events would be required for rejection at $95 \%$ confidence. In contrast, the operator $\mathcal{O}_{5}$ can be distinguished from the standard case even without directional information (owing to its distinctive energy spectrum, which rises with increasing $q^{2}$ ). Including directional information, however, substantially reduces the number of events required for a rejection, with a $5 \sigma$ rejection of SD-only scattering possible with roughly 300 ideal events. This highlights the importance of directional detection in probing the interactions of Dark Matter and distinguishing between different operators, which may not be possible with non-directional experiments alone. 


\section{SEARCHING FOR LIGHT WIMPS}

\section{A. Introduction}

Event rate excesses and annual modulations in various direct detection experiments in recent years have provoked interest in light WIMPs. The CDMS II-Si [216], CoGeNT [217] and CRESST [218] experiments saw excesses above expected backgrounds, while CoGeNT [219] and DAMA/LIBRA [220] observed annual modulations. While these hints could each individually be interpreted as due to the elastic scattering of light $(5-10 \mathrm{GeV})$ WIMPs, there is no model which is consistent with all of the available data (see e.g. Ref. [221]), in particular the recent tight exclusion limits from the LUX experiment [222]. Furthermore, light WIMPs have a strongly declining energy spectrum, with the majority of events lying just above the threshold energy, where background rejection can be difficult. More recent data from CRESST are inconsistent with a light WIMP interpretation of their previous excess [223] and it has been argued that the CoGeNT excess is due to surface (i.e. background) events [224].

Futhermore, light WIMPs are theoretically a viable Dark Matter candidate, as light neutralinos can arise in the Minimal Supersymmetric Standard Model [155, 225, 226], in the Next-to-Minimal Supersymmetric Standard Model (e.g. Ref. [155, 226-228]) and asymmetric Dark Matter (for a review see Ref. [229]).

It is therefore important to pursue experiments that are sensitive to the full range of possible WIMP masses. The differential event rate is quasi-exponential with a characteristic energy scale [14]

$$
E_{\mathrm{c}}=\frac{2 m_{\mathrm{A}} m_{\chi}^{2} v_{\mathrm{c}}^{2}}{\left(m_{\mathrm{A}}+m_{\chi}\right)^{2}} \propto \begin{cases}\frac{m_{\chi}^{2}}{m_{\mathrm{A}}} & \text { if } m_{\chi} \ll m_{\mathrm{A}}, \\ m_{\mathrm{A}} & \text { if } m_{\chi} \gg m_{\mathrm{A}} .\end{cases}
$$

Consequently light targets, such as those typically used in directional detection experiments, are most suited to detecting light WIMPs. Furthermore, as discussed in Sec. VI, directional experiments can probe cross-sections below the neutrino floor, which occurs at $\sigma_{\mathrm{p}}^{\mathrm{SI}} \sim 10^{-42} \mathrm{~cm}^{2}$ for light WIMPs. Below this cross-section non-directional detectors can not distinguish between WIMP induced recoils and those due to ${ }^{8} \mathrm{~B}$ Solar neutrinos, due to their extremely similar energy spectra.

In Sec. X B we review theoretical work examining the potential for directional detection of light WIMPs. Sec. X C then covers experimental prospects. In particular, we evaluate the optimal pressure required for a gaseous directional TPC to be sensitive to light WIMPs. We then discuss the challenges and issues for operating a directional detector at low pressure before concluding in Sec. XD.

\section{B. Theoretical studies}

Theoretical work has demonstrated that directional detection experiments are potentially sensitive to light WIMPs. Morgan and Green [230] determined the number of events and exposures required for a $5 \sigma$ model-independent anisotropy detection of light WIMPs, in the mass range $5-10 \mathrm{GeV}$, for ${ }^{3} \mathrm{He}, \mathrm{C}, \mathrm{F}$ and $\mathrm{S}$ targets with directional energy thresholds in the range $(5-20) \mathrm{keV}$. For vectorial data, where the senses $(+\hat{\mathbf{r}}$ versus $-\hat{\mathbf{r}})$ of all recoils are measured, they used the Rayleigh-Watson statistic [231, 232]. For axial data, they used the Bingham statistic, which is based on the scatter matrix of the data [232]. See Sec.VI for further discussion of these statistics. Apart from the possibility of axial data, they assumed that the recoil directions were perfectly measured in $3 \mathrm{~d}$. Therefore their results provide a lower limit on the number of events/exposure required by a real detector.

They found that between 10 and 200 events are required to reject isotropy, depending on the WIMP and target masses and the energy threshold. The number of events has the advantage of being independent of the (unknown) WIMP elastic scattering cross section. However, as discussed in Sec. VI, quoting sensitives in terms of numbers of events can be misleading. The rare high energy recoils are more anisotropic than the more common low energy recoils (see Fig. 2 in Sec. IV). An experiment with a high energy threshold might only need a small number of events to reject isotropy, however the event rate above a high threshold is small so that a large exposure would be required to accumulate these events. Therefore the exposure provides a more meaningful measure of the amount of data required than the number of events.

For light WIMPs, unless the energy threshold is very low, only the high-speed tail of the WIMP distribution will produce recoils above threshold. As discussed in Sec. III B, there are significant uncertainties in the shape of the high-speed tail of the speed distribution, and in particular the value of the escape speed (above which particles escape from the Milky Way). Therefore for light WIMPs the event rate above threshold can be small and have significant (order of magnitude) uncertainties. Note also that if an experiment is only sensitive to high-speed WIMPs then the Earth's orbital speed must be included, and averaged over, for an accurate calculation of the event rate [230]. 
Consequently Ref. [230] found that the exposure necessary to accumulate the $10-200$ events required to reject isotropy depends strongly on the energy threshold and the target nuclei and WIMP masses. For a spin independent elastic scattering cross-section on the proton of $\sigma_{\mathrm{p}}^{\mathrm{SI}}=10^{-5} \mathrm{pb}$ the exposure required varies between $10^{2}$ and $>$ $10^{7} \mathrm{~kg}$ day. If the minimum speed required to cause a recoil above threshold, $v_{\min }\left(E_{\mathrm{th}}\right)$, is significantly smaller than the maximum WIMP speed in the lab frame, $v_{\max }$, then the exposure required is fairly modest, $\sim 10-100 \mathrm{~kg}$ day. This is the case for a low energy threshold and the larger WIMP and target masses considered. However as the energy threshold is increased, and $v_{\min }\left(E_{\mathrm{th}}\right)$ approaches $v_{\max }$, the event rate above threshold drops rapidly and the exposure required increases substantially. The shape of the high-speed tail of the velocity distribution, and in particular the value of the escape speed, also have a significant effect on the required exposure [230].

\section{Experimental prospects}

In this sub-section we consider the experimental possibilities, and requirements, for a directional low mass WIMP search. The usual challenges for a Dark Matter experiment probing this regime are exacerbated for directional searches that, in addition, require tracking of the very low energy recoils. The most mature current directional experiments are all based on the low-pressure gas TPC technology operating at pressures of $\sim 40-100$ Torr, which are optimal for $m_{\chi} \sim 100 \mathrm{GeV}$ WIMPs. At these pressures, however, recoil tracks from low mass WIMPs are too short to be resolved, resulting in little or no sensitivity in the $m_{\chi} \approx 10 \mathrm{GeV}$ region. Nevertheless, with the recent interest in low mass WIMPs, a number of authors [233, 234] have studied the question of whether appropriate optimization of the experiments can lead to directional sensitivity.

Gas-based TPCs, with their ability to vary the target gas and its pressure, are ideally suited to address this question. As discussed in Sec. X A, due to the kinematics of elastic scattering the optimal target nuclei mass is of order the WIMP mass. Fortunately there exist numerous drift chamber gases, such as $\mathrm{CO}_{2}, \mathrm{CF}_{4}$ and $\mathrm{CS}_{2}$, with nuclei ideally suited as light WIMP targets. Additionally, low mass WIMP searches require the directional energy threshold to be as low as possible due to the steeply falling recoil energy spectrum. The possibility of gas-based TPCs to operate at arbitrarily low pressures could provide the ability to lengthen recoil tracks of a given energy, thereby lowering the directional energy threshold for a fixed minimum resolvable track length, $R_{\min }$. The minimum resolvable track length is a characteristic of the experimental properties of the detector and can be determined by simulations or direct measurements.

Given $R_{\min }$, the optimal pressure can be derived by maximizing the directional event rate. The existence of an optimal pressure is due to two competing effects that conspire to give a maximum directional event rate. As the pressure is increased both the target mass and the directional threshold increase. The former increases the event rate linearly, while the latter decreases it exponentially, resulting in an optimal pressure where the rate peaks. Martoff et al. [233] used simulations for $R_{\min }$ values in the range $0.1-10 \mathrm{~mm}$ to find optimal pressures for $\mathrm{Ar} \mathrm{CF}_{4}, \mathrm{CS}_{2}$ and Xe for WIMP masses in the range 10 to $1000 \mathrm{GeV}$. Jaegle et al. [234] first ran simulations to determine $R_{\text {min }}$ as a function of pressure in $\mathrm{CF}_{4}$ and and $\mathrm{CS}_{2}$, then followed a similar procedure to find the optimal pressure. Ref. [233] predict a few events per year per cubic metre for $m_{\chi} \approx 10 \mathrm{GeV}$ and $\sigma_{\mathrm{p}}^{\mathrm{SI}} \approx 10^{-42} \mathrm{~cm}^{2}$, while Ref. [234] finds a slightly smaller rate.

Phan et al. [235] follow a similar procedure to optimize pressures for light WIMPs, except that their $R_{\text {min }}$ is determined from experimental data. The data were taken using a high resolution, high signal-to-noise prototype TPC that measures two dimensional tracks in 100 Torr $\mathrm{CF}_{4}$. With a diffusion of $\sigma \approx 0.4 \mathrm{~mm}$, not unreasonable for negativeion TPCs using $\mathrm{CS}_{2}$ gas mixtures [236], they measure $2 \mathrm{~d}$ vector directionality down to track lengths $\sim 0.6 \mathrm{~mm}$, which corresponds to a $55 \mathrm{keV}_{\mathrm{r}}$ fluorine recoil in 100 Torr $\mathrm{CF}_{4}$. For axial directionality (i.e. with no measurement of the sense of the recoil) their minimum resolvable track size is $\sim 0.4 \mathrm{~mm}$, corresponding to $\approx 40 \mathrm{keV}$. Here we use their result of $R_{\min }=0.6 \mathrm{~mm}$ for $2 \mathrm{~d}$ vector directionality to find the optimal pressure using the procedure described above.

The directional rate as a function of pressure is shown in Fig. 19 for three gases, $\mathrm{CO}_{2}, \mathrm{CF}_{4}$ and $\mathrm{CS}_{2}$, for a WIMP with $m_{\chi}=10 \mathrm{GeV}$ and $\sigma_{\mathrm{p}}^{S I}=10^{-42} \mathrm{~cm}^{2}$. These plots show that to detect light WIMPs a directional detector needs to operate in the $5-20$ Torr range depending on the choice of light target. The corresponding directional energy threshold is in the range $5-10 \mathrm{keV}_{\mathrm{r}}$. The resulting maximum rates are consistent with those derived in Ref. [234] but systematically lower than in Ref. [233]. The top right panel of Fig. 19 show how the optimal pressure depends on the minimum resolvable track length, for $0.3 \mathrm{~mm}<R_{\min }<1.5 \mathrm{~mm}$, and WIMP masses in the range $5-30 \mathrm{GeV}$. These studies show that several cubic metre scale directional experiments, if appropriately configured with high resolution readouts, and operating with low diffusion, optimized pressures and targets, can probe new parameter space for low mass WIMPs.

We now describe some of the experimental challenges and steps needed to operate a directional detector at low pressure and low energy threshold, as required for a low mass WIMP search. The first set of challenges are related to the practical issues of operating gaseous TPCs at the low pressure, $<20$ Torr, see Fig. 19. The requirement of 
high voltages needed for maintaining the drift field in the TPC, and for achieving high gas gains in the amplification device, is incompatible with stable operation at low pressures. Nevertheless, high gas gains in micro-patterned gas detectors such as Thick GEMs (THGEMs) have been demonstrated down to $\sim 5$ Torr in certain gases [237]. Using such devices, Phan et al. [235] have shown sufficiently high gas gains with $\mathrm{CS}_{2}$ close to 10 Torr. Stable high voltage operation for sustaining a high drift field over tens of $\mathrm{cm}$ remains to be demonstrated. Another practical challenge at these low pressures is maintaining the low diffusion that was critical for Phan et al to achieve their result of $R_{\min } \approx 0.4-0.6 \mathrm{~mm}$. Whether the thermal diffusion characteristic of negative ion gases, such as $\mathrm{CS}_{2}$, extends to low pressures also needs to be tested.

The second set of challenges are more fundamental in that they relate to the physics that characterizes the track structure of very low energy nuclear recoils. The above extrapolation relies on the assumption that a $1 \mathrm{~mm}$ long nuclear recoil track at high pressure is essentially the same as a lower energy, $1 \mathrm{~mm}$ long track at low pressure. There are a number of reasons why this might not be true. One is that, at low energies, there is more straggling (fluctuations in energy-loss and range) and larger deviation in the recoil's trajectory from its initial direction due to multiple scattering. In addition, the asymmetry in the ionization along the track, which gives rise to the head-tail effect, could be weaker at low energies. Finally, the fraction of energy loss into ionization (quenching) is expected to decrease at lower energies, which reinforces the need to achieve high gas gains at low pressures. Given the steeply falling energy spectrum from low mass WIMPs, all of these effects could strongly dilute their directional signal relative to that observed at higher energies in the 100 Torr data from Ref. [235]. Addressing these issues will require experimental data at low pressures, and will be critical for determining the exposure needed for the directional discovery of low mass WIMPs.

The third challenge is related to background discrimination, which also needs to extend down to the $5-10 \mathrm{keV}_{\mathrm{r}}$ range required for directionality. For nuclear recoil energy thresholds in this range the detected energy would be in the range $2-4 \mathrm{keV}_{\mathrm{ee}}$, or even lower, since quenching factors are not yet measured at these energies. Thus, experimental data are also needed to investigate discrimination at these low energies, as well as studies of the background rates in the large detectors required.

\section{Conclusions}

In this section we have discussed the theoretical and experimental challenges for the directional detection of light WIMPs. In principle, with an ideal detector, only $10-100$ events are required to detect an anisotropic WIMP signal. However for light WIMPs only high speed WIMPs in the tail of the speed distribution, can cause nuclear recoils which are long enough for their direction to be measured. The event rate above threshold is therefore typically small, and hence the exposure required to detect a signal can be large. Furthermore there are significant uncertainties in the shape of the high speed tail of the WIMP distribution which lead to significant uncertainties in the event rate above threshold, and consequently the required exposure.

Most directional detectors use gaseous TPCs operating at $\sim 100$ Torr. However at this pressure the track lengths of the low energy nuclear recoils induced by light WIMPs are too short to be resolved. We have overviewed results from simulations of TPCs at 10 Torr and also extrapolations of data from a prototype 2 d detector operated with $\mathrm{CF}_{4}$ at 100 Torr. These studies indicate that cubic metre scale low pressure TPCs could measure the directions of nuclear recoils from light WIMPs and probe new regions of WIMP mass-cross-section parameter space. Finally we discussed the challenges for a directional light WIMP search in the required low pressure, $10-20$ Torr, regime. Namely the practical challenges in operating a TPC at low pressure, and the uncertainties in the physics of low energy nuclear and electron recoils at low pressures and energies.

\section{PROBING HALO SUBSTRUCTURE}

\section{A. Introduction}

The use of directional detection to constrain the astrophysical properties of the Dark Matter velocity distribution is an exciting possibility. Directional detection may not only constrain the properties of the halo velocity distribution $[157,158]$ (see Sec. VIII), but may also be sensitive to the presence of velocity substructures in the halo. As discussed in Sec. III B, simulations suggest that these substructures might include Dark Matter tidal streams (spatially localized), debris flows (spatially homogenized), and a co-rotating dark disk. Such components of the local velocity distribution can lead to distinctive features in the directional signal [128, 158, 183, 215, 238]. However, the detectability of these features depends strongly on the unknown properties of the substructure - as does the possibility of constraining these properties - and may require a very low energy threshold and/or a large exposure. In the following, we review the 

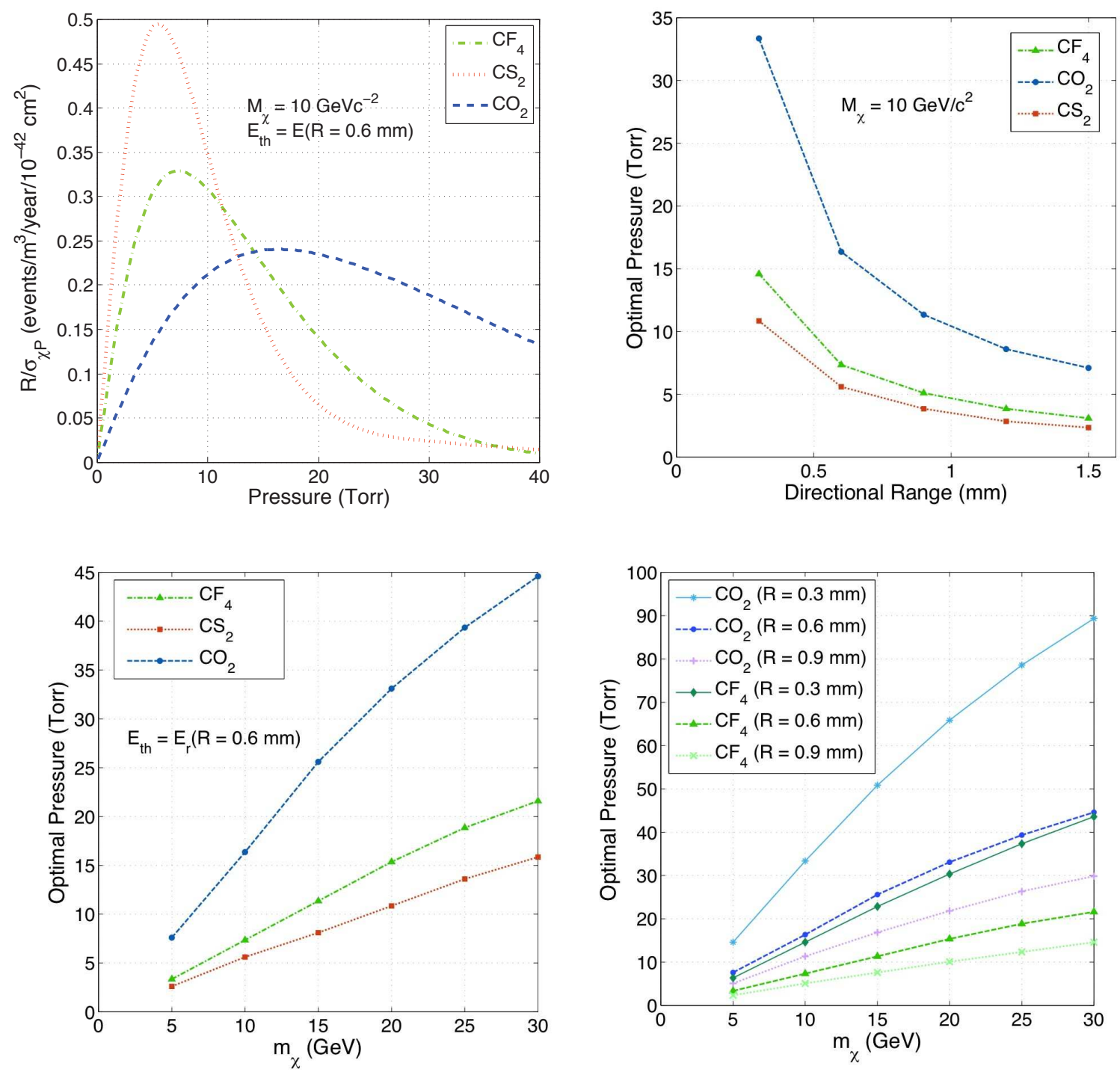

FIG. 19: Top left panel: The total directional rate, per cubic metre, per year, for a WIMP with mass $m_{\chi}=10 \mathrm{GeV}$ and spin-independent cross-section $\sigma_{\mathrm{p}}=10^{-42} \mathrm{~cm}^{2}$ for $\mathrm{CF}_{4}$ (green dashed line), $\mathrm{CS}_{2}$ (red dotted) and $\mathrm{CO}_{2}$ (blue dotted) as a function of pressure for a minimum track length, $R_{\min }=0.6 \mathrm{~mm}$. Top right: the optimal pressure as a function of $R_{\mathrm{min}}$, for the same gases and WIMP mass. Bottom left: the optimal pressure as a function of WIMP mass, with $R_{\mathrm{min}}=0.6 \mathrm{~mm}$, and bottom right, the same but with 3 different values of $R_{\min }$, as indicated, and for $\mathrm{CO}_{2}$ and $\mathrm{CF}_{4}$ only.

sensitivity of directional detection to these velocity substructures, focusing on Dark Matter streams in Sec. XIB and a dark disk in Sec. XI C.

\section{B. Streams}

As discussed in Sec. III B, tidal streams are a class of substructures caused by the disruption and accretion by the Milky Way of stars and Dark Matter from satellite galaxies. The highly localised and anisotropic nature of tidal streams in both position and velocity make them an intriguing prospect for directional detection. A tidal stream such as this in the local Galactic region would manifest itself in the directional signal as a ring feature with increasing angular radius at lower energies (see Sec. IV). Streams produce step-like features to the non-directional energy spectrum [131], as well as non-sinusoidal components to the annual modulation signal [239]. 
The simplest way to incorporate substructure into a halo model is by combining two parameterized distributions, a background distribution for the smooth halo and an additional component corresponding to the substructure. When necessary, we shall adopt the Maxwell-Boltzmann velocity distribution for the smooth halo, as deviations from this are not likely to have an appreciable effect on the detection of streams. We further assume that a single tidal stream makes up a fraction $A_{S}=\rho_{\text {str }} / \rho_{0}$ of the local Dark Matter density, so that the total velocity distribution of the halo+stream model is given by

$$
f_{\mathrm{g}}^{\mathrm{h}+\mathrm{s}}(\mathbf{v})=\left(1-A_{S}\right) f_{\mathrm{g}}^{\text {halo }}\left(\mathbf{v} ; \sigma, v_{\mathrm{esc}}\right)+A_{S} f_{\mathrm{g}}^{\mathrm{str}}\left(\mathbf{v} ; \mathbf{v}_{\mathrm{str}}, \sigma_{\mathrm{str}}, v_{\mathrm{esc}}\right),
$$

where,

$$
\begin{aligned}
f_{\mathrm{g}}^{\text {halo }}\left(\mathbf{v} ; \sigma, v_{\mathrm{esc}}\right) & =f_{\mathrm{g}}^{\mathrm{MB}}\left(\mathbf{v} ; \sigma, v_{\mathrm{esc}}\right) \\
f_{\mathrm{g}}^{\mathrm{str}}\left(\mathbf{v} ; \mathbf{v}_{\mathrm{str}}, \sigma_{\mathrm{str}}, v_{\mathrm{esc}}\right) & =f_{\mathrm{g}}^{\mathrm{MB}}\left(\mathbf{v}-\mathbf{v}_{\mathrm{str}} ; \sigma_{\mathrm{str}}, v_{\mathrm{esc}}\right) .
\end{aligned}
$$

For simplicity, we have taken the velocity distribution of the stream to also be a Maxwell-Boltzmann distribution, but with dispersion $\sigma_{\text {str }}$ and a mean velocity $\mathbf{v}_{\text {str }}$ in the Galactic frame.

The full Radon transform for the halo+stream model is therefore

$$
\hat{f}_{\mathrm{e}}^{\mathrm{h}+\mathrm{s}}\left(v_{\min }, \hat{\mathbf{r}}\right)=\left(1-A_{S}\right) \hat{f}_{\mathrm{g}}^{\mathrm{MB}}\left(v_{\min }+\mathbf{v}_{\mathrm{e}} \cdot \hat{\mathbf{r}}, \hat{\mathbf{r}} ; \sigma, v_{\mathrm{esc}}\right)+A_{S} \hat{f}_{\mathrm{g}}^{\mathrm{MB}}\left(v_{\min }+\left(\mathbf{v}_{\mathrm{e}}-\mathbf{v}_{\mathrm{str}}\right) \cdot \hat{\mathbf{r}}, \hat{\mathbf{r}} ; \sigma_{\mathrm{str}}, v_{\mathrm{esc}}\right),
$$

where $\hat{f}_{\mathrm{g}}^{\mathrm{MB}}$ is the Radon transform of the Galactic frame Maxwell-Boltzmann distribution. The recoils originating from scattered stream WIMPs are only dependent on the direction of the stream through the angle between the stream velocity and Earth's velocity. Hence, the dependence of the recoil distribution on the stream velocity is completely determined by the stream speed $v_{\text {str }}$ and the angle

$$
\Delta \theta=\cos ^{-1}\left(\hat{\mathbf{v}}_{\mathrm{e}} \cdot \hat{\mathbf{v}}_{\mathrm{str}}\right)
$$

Using this halo+stream model to simulate recoil events, we can investigate the ability of directional detectors to characterize tidal streams. There are two steps in characterizing a stream using directional detection. The stream must first be detected; its parameters, e.g. density and velocity, can then be measured. Thus, we first consider statistical tests of stream detectability (summarizing results from Ref. [240]), and then discuss parameter estimation of stream properties (summarizing Ref. [158]). The model detector used throughout this section is an idealized MIMAC-like detector with a $5 \mathrm{keV}$ threshold. In the case of the parametric test a pixelization of a few degrees was used as a proxy for angular resolution.

\section{Stream detectability}

We first discuss non-parametric statistical tests of stream detectability. Using only directional information, these tests can determine whether features inconsistent with a completely isotropic halo can be detected for streams, such as those with velocity distributions given by Eqs. (34-35). Note, however, that they do not require that the form of the stream velocity distribution is known a priori. Such tests have been implemented in previous studies to determine the number of events required to distinguish a WIMP signal from isotropic backgrounds [70], see Sec. VI. One advantage of a non-parametric analysis is that it is also not necessary to assume a particular model for the smooth component of the halo. The results are valid provided the basic hypotheses that define the statistical tests are satisfied. As such, the results here are valid for any background halo model that is isotropic in the Galactic frame. However, a notable disadvantage of such a non-parametric analysis is that it is less powerful than a parametric analysis (in the sense that, for a given data set, the detection significance will be lower). Hence, we will then consider the use of a profile likelihood ratio test to detect the presence of a stream parametrically. In this case, the choice of background halo model (and the number of parameters needed to specify it) will affect the shape of the likelihood function; again, however, the effect of deviations from our choice of a simple Maxwell-Boltzmann halo is likely to be small.

In the following results, shown in Fig. 20, the analysis was performed on simulated MIMAC-like experiments with a ${ }^{19} \mathrm{~F}$ target. The recoil sets for each Monte-Carlo experiment were generated assuming an exposure of $10 \mathrm{~kg}$ yr and a spin dependent WIMP-proton cross section of $\sigma_{p}=10^{-3} \mathrm{pb}$ (just below the exclusion limit set by XENON100 [241]). The local WIMP density was set to its canonical value $0.3 \mathrm{GeV} / \mathrm{cm}^{-3}$, and the WIMP mass was taken to be $50 \mathrm{GeV}$. For the background halo parameters, the values were set at $v_{\text {esc }}=533 \mathrm{~km} \mathrm{~s}^{-1}[100]$ and $\sigma=220 / \sqrt{2} \mathrm{~km} \mathrm{~s}{ }^{-1}$. The stream velocity dispersion was set to $\sigma_{\mathrm{str}}=10 \mathrm{~km} \mathrm{~s}^{-1}$ and its density fraction was set to a slightly large $A_{S}=0.1$ (simulations suggest that typical tidal streams are more likely at the $1 \%$ level $[115,242]$ ), but the results hold for more realistic stream fractions if correspondingly larger exposure times are assumed. The effects of annual modulation have 


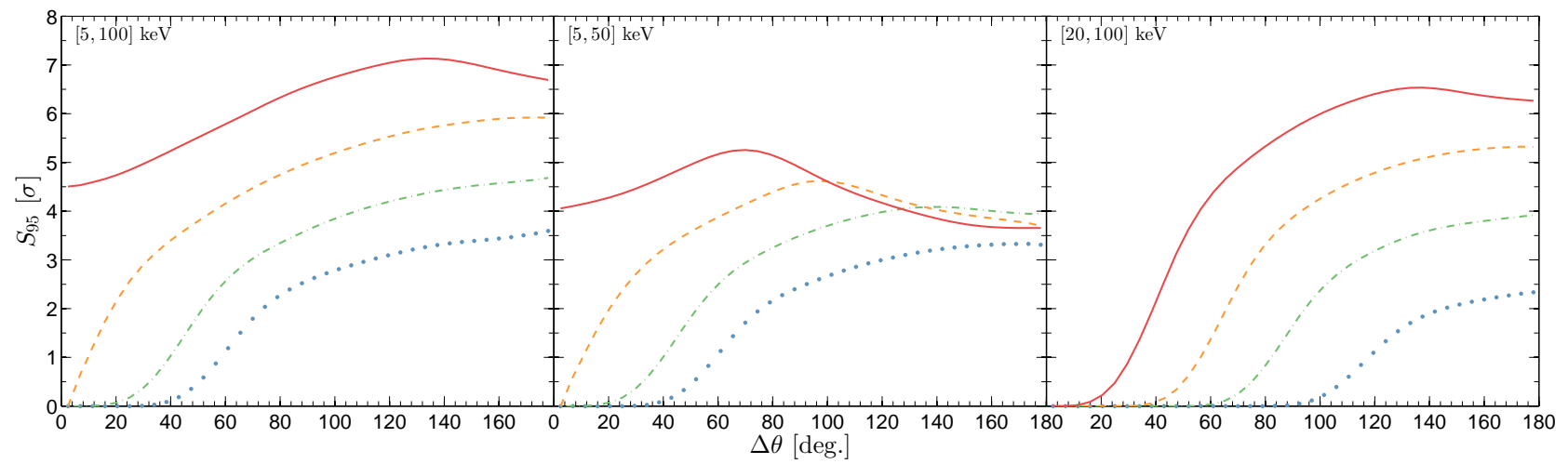

FIG. 20: The significance obtainable by $95 \%$ of idealized experiments, $S_{95}$, in units of $\sigma$, as a function of the angle $\Delta \theta$ between the Earth and stream velocities. From top to bottom in each panel, the curves correspond to $v_{\mathrm{str}}=500 \mathrm{~km} \mathrm{~s}{ }^{-1}$ (red solid), 400 $\mathrm{km} \mathrm{s}^{-1}$ (orange dashed), $300 \mathrm{~km} \mathrm{~s}^{-1}$ (green dot-dashed), $200 \mathrm{~km} \mathrm{~s}^{-1}$ (blue dotted). The three panels are for energy windows $[5,100] \mathrm{keV},[5,50] \mathrm{keV}$ and $[20,100] \mathrm{keV}$, from left to right.

been ignored in the following results. The experimental backgrounds were generated from an isotropic, energy-flat distribution with a rate of $R_{\mathrm{b}}=10 \mathrm{~kg}^{-1} \mathrm{yr}^{-1}$.

\section{Non-parameteric tests}

The non-parametric tests we consider consist of extracting a statistic that is distributed according to some null distribution in the case that the halo is a smooth and isotropic distribution $\left(A_{S}=0\right)$, and is distributed as an alternative distribution (which can be Monte-Carlo generated using mock data) in the case that the halo possesses an additional stream component $\left(A_{S} \neq 0\right)$. A smooth and isotropic halo model will produce a directional signal that has a median direction that corresponds to the Earth's velocity, as well as rotational symmetry around this direction. There are well defined procedures, which are outlined in detail in Ref. [240], that can be followed to test for these two hypotheses. To test the median direction, a $\chi^{2}$ statistic with a distribution that asymptotes to a $\chi_{2}^{2}$ distribution in the null case is extracted. To test the rotational symmetry, we use a modified Kuiper statistic $V^{\star}$; in the null case, the distribution of this statistic has no analytic form, but can be built by performing the test on any data set with rotational symmetry about the hypothesized direction (e.g., isotropic experimental backgrounds). The tests do not perform equally for all stream directions. In particular, the tests return a low significance when the stream is anti-aligned with the Earth velocity, as in this case the null hypotheses of rotational symmetry and inverse-Earth median direction are in fact correct. The number of events from stream WIMPs also plays an important role in determining the significance which can be achieved. For streams with small $\Delta \theta$, this number is very low, and for low stream speeds or high threshold energies it can even be zero.

\section{Parametric tests}

We now consider parametric tests of detectability, assuming that the velocity distribution of the halo+stream is indeed given by the parameterized function described by Eqs. (34-35). The profile likelihood ratio between the null (no stream) and alternative (stream) hypotheses is

$$
\Lambda=\frac{\mathcal{L}\left(\hat{\hat{\boldsymbol{\theta}}}, A_{S}=0\right)}{\mathcal{L}(\hat{\boldsymbol{\theta}})},
$$

where $\hat{\boldsymbol{\theta}}$ are the maximum likelihood estimators for the WIMP and halo parameters in the alternative model, and $\hat{\hat{\boldsymbol{\theta}}}$ are the maximum likelihood estimators evaluated when the stream density fraction is zero, $A_{S}=0$. The profile likelihood ratio test statistic is then defined as

$$
\mathcal{D}=\left\{\begin{aligned}
-2 \ln \Lambda & 0 \leq \hat{A}_{S} \leq 1 \\
0 & \hat{A}_{S}<0, \hat{A}_{S}>1
\end{aligned}\right.
$$

The advantage of this test for the detection of streams is that it assumes the null hypothesis is contained within the alternative hypothesis under a certain condition $\left(A_{S}=0\right)$. Then, according to Wilk's theorem [243], the distribution of the test statistic when the null hypothesis is true becomes a $\chi_{1}^{2}$ distribution, so the significance of a particular result $\mathcal{D}_{o}$ can be written in units of $\sigma$ as $S=\sqrt{\mathcal{D}_{o}}$. 
Fig. 20 shows the detection significance obtainable for $95 \%$ of experiments that is inferred with this test, assuming exposure times of $5 \mathrm{~kg}$ yr. The test, by virtue of being parametric, performs much better than the non-parametric tests. For example, for a stream with mid-range parameter values (a speed of $300 \mathrm{~km} \mathrm{~s}^{-1}$ and an angle of $90^{\circ}$ ), the tests for the three energy windows detect the stream at $4-5 \sigma$ in $95 \%$ of experiments. For the same exposure, the non-parametric tests only reach a value of $S_{95}$ between 0.1 and 0.2 [240]. As in Ref. [158], the enhancement in performance is also due to the use of the full energy and direction information, as opposed to the use of only the latter in the non-parametric tests. Furthermore, the parametric tests achieve high significance over a wide range of stream velocities, with the limiting factor being the number of WIMPs coming from the stream. For low values of $\Delta \theta$, where the number of stream WIMPs drops to zero, the significance can be seen to do likewise. There is similarly a dependence on the energy window of the detector, which causes a reduction in the number of stream WIMPs when the stream becomes boosted such that the recoils fall past the maximum of the energy window. This can be seen clearly in the $[5,50] \mathrm{keV}$ case. For a stream speed of $500 \mathrm{~km} \mathrm{~s}^{-1}$, the significance begins to decrease for $\Delta \theta>70^{\circ}$, and drops by $1.6 \sigma$ up to $\Delta \theta=180^{\circ}$. However, the significance for faster stream speeds is enhanced over what might be expected simply from the dependence on the number of stream WIMPs. This is due to faster streams becoming more prominent because of the exponential drop off with energy of the event rate for the smooth halo.

To summarise, the results described here demonstrate that there is reasonable prospect for detecting streams in the local Galactic region. However the detection significance achievable is highly dependent on the speed and direction of the stream, with slow streams aligned with the Earth's velocity evading detection in both non-parametric and likelihood analyses. The threshold and maximum energy of the detector also restrict the detection significance as they exclude recoils scattering from the stream WIMPs for very slow and very fast streams.

\section{Constraining stream parameters}

Directional-detection experiments may also be able to reconstruct the parameters of a stream [158, 240]. Likelihoodbased methods $[158,183]$ used to estimate the parameters of the halo velocity distribution can be easily extended to models with substructure, if the substructure velocity distributions can also be characterized by parameterized functional forms - as we have done for the case of a stream in Eqs. 34-35. Here, 5 parameters characterize the stream: the stream fraction $A_{S}$, its direction in Galactic coordinates $\left(\ell_{S}, b_{S}\right)$, its speed $v_{S}$, and its velocity dispersion $\sigma_{S}$. Combining these with parameters that describe the smooth halo, the WIMP mass, and the fraction $\lambda$ (defined by Eq. 2), Ref. [158] considered a halo+stream model with a total of 10 parameters. In particular, $A_{S}=0.1, v_{S}=510$ $\mathrm{km} \mathrm{s}^{-1}$, and $\sigma_{S}=10 \mathrm{~km} \mathrm{~s}^{-1}$ were taken for the stream parameters, while typical values were assumed for the smooth halo.

Assuming a WIMP mass of $50 \mathrm{GeV}$, a spin dependent WIMP-proton cross section of $\sigma_{p}=10^{-3} \mathrm{pb}$, and a $30 \mathrm{~kg}$ year exposure for a $C F_{4}$ detector with a 5 -keV threshold, a corresponding $\sim 650$ signal and $\sim 300$ background events were simulated (giving a signal fraction $\lambda \approx 68 \%$ ). Bayesian parameter estimation was performed on this simulated data set, leading to the posterior probability distributions presented in Fig. 21. For all parameters, reconstructed values are consistent with their input values. However, the constraint on the WIMP mass is relatively poor. Despite this, the stream parameters (bottom row of Fig. 21) are recovered with fairly good accuracy. This parameter-estimation analysis, along with a similar one carried out in Ref. [240], thus demonstrates the possibility of using directional detectors to measure the properties of tidal streams. Finally it is worth mentioning that although this analysis used a pixelisation of the angular data to provide a proxy for angular resolution, a full treatment of angular smearing would be required to determine how the constraints on stream parameters were affected by a finite angular resolution. However since the angular spread of stream WIMP induced recoils is rather large provided the angular resolution is below a few tens of degrees the stream is likely to be resolved relative to the background halo recoils.

\section{Dark disk}

\section{Constraining dark-disk parameters}

The ability of directional-detection experiments to measure the properties of a dark disk was also discussed in Ref. [158]. As mentioned in Sec. III B, simulations suggest that subhalos merging with the Milky Way may have been disrupted by the baryonic disk, leading to the formation of a co-rotating dark disk. A parameterized velocity distribution for a halo+disk model can be constructed as in the case of a halo+stream, by simply replacing the stream parameters with the corresponding disk parameters. Parameter estimation of simulated data sets can then be performed identically. 


\section{DECOMPOSING THE VELOCITY DISTRIBUTION}

\section{A. Introduction}

In Sec. XI, we reviewed the sensitivity of directional-detection experiments to halo and substructure velocity distributions that could be described by functional forms with a small number of parameters. Three recent studies have shown how to go beyond the assumption of such velocity distributions. The first expands the velocity distribution in terms of moments of the integrals of motion [244] (which is possible if certain equilibrium conditions hold). The second and third use Fourier-Bessel expansion [245] and angular discretisation [246], which allow for arbitrary velocity distributions. These expansions provide a general framework for a detailed characterization of the velocity distribution using directional detection in the post-discovery era, which could be possible once a sufficiently large number of events have been observed.

\section{B. Moment decomposition}

If the majority of Dark Matter particles in the halo have reached a state of equilibrium, Jeans' Theorem holds to a good approximation [99]. It states that any function of the integrals of motion is a steady-state solution of the collisionless Boltzmann equation. This allows us to infer global properties of the Dark Matter halo from an estimation of the integrals of motions in the solar neighborhood. Frollowing the strong version of the Jeans' theorem, three integrals of motion have to be chosen. The choice made in Ref. [244] is as follows:

- The energy $\mathcal{E}$ given by $\mathcal{E}=\frac{v^{2}-v_{\text {esc }}^{2}}{2}$.

- The $z$-component of the angular momentum, $L_{z}$ that is equal to $r_{\oplus} v_{\phi}$ in the solar neighborhood, $\phi$ being the azimuthal angle with respect to the orbit of the Earth.

- The magnitude of the angular momentum $L_{t}$ given by $L_{t}=r_{\oplus} v_{\theta}$ in the Solar neighborhood, $\theta$ being the angle between the velocity vectors of the recoil and of the Earth's motion.

The assumption is made that $f\left(\mathcal{E}, L_{t}, L_{z}\right)$ is separable, i.e. $f\left(\mathcal{E}, L_{t}, L_{z}\right)=f_{1}(\mathcal{E}) f_{2}\left(L_{t}\right) f_{3}\left(L_{z}\right)$ and the distribution functions of each of the integral of motion are given by the following special function decompositions

$$
\begin{aligned}
f_{1}(\mathcal{E}) & =\sum_{\ell} c_{P_{\ell}} \tilde{P}_{\ell}\left(\frac{\mathcal{E}}{\mathcal{E}_{\lim }}\right), \\
f_{2}\left(L_{t}\right) & =\sum_{n} c_{F_{n}}^{t} \cos \left(n \pi \frac{L_{t}}{L_{\max }}\right), \\
f_{3}\left(L_{z}\right) & =\sum_{m} c_{F_{m}}^{z} \cos \left(m \pi \frac{L_{z}}{L_{\max }}\right),
\end{aligned}
$$

where $\tilde{P}_{\ell}$ are shifted Legendre polynomials, $\mathcal{E}_{\text {lim }}$ is the lowest Dark Matter energy corresponding to the recoil threshold $E_{r}^{\text {th }}$ and $L_{\max } \equiv r_{\oplus} v_{\text {esc }}$.

This parametrization has previously been applied to mock data, simulated for a background-free $\mathrm{CS}_{2}$ directional detector, with recoil energy between 5 and $15 \mathrm{keV}$ and for a $6 \mathrm{GeV}$ WIMP. The double-differential spectrum $\mathrm{d}^{2} R / \mathrm{d} E_{r} \mathrm{~d} \Omega_{r}$, from this simulated data, is then used to reconstruct the decomposition coefficients in order to infer $f_{1}(\mathcal{E}), f_{2}\left(L_{t}\right)$, and $f_{3}\left(L_{z}\right)$ and hence $f\left(\mathcal{E}, L_{t}, L_{z}\right)$.

Ref. [244] thus demonstrated that a few thousand events are sufficient to allow for a good measurement of the Dark Matter distribution. The method was applied to a distribution described by the analytic Michie distribution [247], as well as to a distribution extracted from the Via Lactea II N-body simulation [248]. In the latter case, the input model contains anisotropies and departures from a standard Maxwellian distribution [118]. Within error bars, the distributions of integrals of motion that are evaluated from the directional data are in very good agreement with the input Dark Matter distribution, indicating that this approach can accommodate such deviations from the SHM. The evaluation of the integrals of motion at a given radius may then be used to infer the Dark Matter distribution at any radius, providing the distribution is in a steady state of equilibrium. This has been checked on data from the Via Lactea II simulation up to larger radii $(30 \mathrm{kpc})$.

In conclusion, directional detection may be used to infer Dark Matter phase space distribution in the solar neighborhood, through an evaluation of the coefficients of its moment decomposition on a model independent basis. As 
noted in Ref. [244], this method avoids the use of an analytic Dark Matter model and is hence one step toward a model independent analysis of directional data. However, it relies on the assumption of equilibrium and also requires that the WIMP mass is known a priori, and can therefore only be applied in the post-discovery era.

\section{Fourier-Bessel decomposition}

The moment decomposition requires that the WIMP velocity distribution is a separable function of the integrals of motion. If the velocity distribution cannot be represented as such, it may then be necessary to work in a basis that allows for arbitrary velocity distributions. This may be the case if the Dark Matter halo contains a significant unvirialized fraction.

One possible basis is given by a generalization of the Fourier-Bessel basis. Since the Galactic-frame WIMP velocity distribution $g(\mathbf{v})$ is typically truncated at the escape velocity $v_{\text {esc }}$, we consider basis functions that are given by the product of a spherical Bessel function of the first kind $j_{l}$ and a real spherical harmonic $S_{l m}$, and which vanish for $v>v_{\text {esc }}$ :

$$
\Psi_{l m}^{n}(\mathbf{v})=4 \pi c_{l n} j_{l}\left(u_{l n} v\right) S_{l m}(\hat{\mathbf{v}}) \theta\left(v_{\mathrm{esc}}-v\right)
$$

These basis functions are hence labeled by integers $n, l$, and $m$. Here, $u_{l n}=x_{l n} / v_{\text {esc }}$, where $x_{l n}$ is the $n$th zero of $j_{l}$, and $c_{l n}^{-1} \equiv x_{l n} j_{l+1}\left(x_{l n}\right) / \sqrt{\pi}$. An arbitrary velocity distribution truncated at $v_{\text {esc }}$ can then be expanded as

$$
g(\mathbf{v})=\sum_{n l m} \frac{x_{l n}^{2} v_{\mathrm{esc}}^{-3}}{(2 \pi)^{3}} \Psi_{l m}^{n}(\mathbf{v}) g_{l m}^{n}
$$

and is completely characterized by the expansion coefficients $g_{l m}^{n}$. Note that the requirement that $g(\mathbf{v})$ is positive definite places a restriction on these coefficients.

Besides the ability to represent arbitrary velocity distributions, another advantage of the Fourier-Bessel basis is that it also admits an analytic expression for the directional recoil spectrum $\mathrm{d}^{2} R / \mathrm{d} E_{r} \mathrm{~d} \Omega_{\mathrm{r}}$ in terms of the expansion coefficients $g_{l m}^{n}$. A detailed derivation of this analytic expression can be found in Ref. [245]; we simply summarize the result here.

The directional recoil spectrum $\mathrm{d}^{2} R / \mathrm{d} E_{r} \mathrm{~d} \Omega_{\mathrm{r}}$ is easily found given the Radon transform $\widehat{f}$ of the lab-frame velocity distribution $f$. In turn, because the lab-frame distribution $f$ and the Galactic-frame distribution $g$ are simply related by a translation in velocity space, using the translation properties of the Radon transform we can easily find $\widehat{f}$ given $\widehat{g}$. Furthermore, because of the linearity of the Radon transform, we see that the expansion in Eq. (43) yields

$$
\widehat{g}\left(v_{\mathrm{r}}, \hat{\mathbf{u}}\right)=\sum_{n l m} \frac{u_{l n}^{2} v_{\mathrm{esc}}^{-1}}{(2 \pi)^{3}} \widehat{\Psi}_{l m}^{n}\left(v_{\mathrm{r}}, \hat{\mathbf{u}}\right) g_{l m}^{n} .
$$

Thus, the net implication is that the directional recoil spectrum $\mathrm{d}^{2} R / \mathrm{d} E_{r} \mathrm{~d} \Omega_{\mathrm{r}}$ can be easily computed if the coefficients $g_{l m}^{n}$ and the Radon transforms $\widehat{\Psi}_{l m}^{n}$ of the basis functions are known.

The crux of the derivation is then the calculation of $\widehat{\Psi}_{l m}^{n}$, which can be accomplished by contour integration and the utilization of various mathematical properties of both the Fourier-Bessel basis functions and the Radon transform. The result is

$$
\widehat{\Psi}_{l m}^{n}\left(v_{\mathrm{r}}, \hat{\mathbf{u}}\right)=8 \pi v_{\mathrm{esc}}^{2} i^{-l} c_{l n} x_{l n} j_{l-1}\left(x_{l n}\right) K_{l}^{n}\left(v_{\mathrm{r}} / v_{\mathrm{esc}}\right) S_{l m}(\hat{\mathbf{u}})
$$

where we have defined the integrals

$$
K_{l}^{n}(a)=\int d x \frac{e^{i a x} j_{l}(x)}{x^{2}-x_{l n}^{2}}
$$

This integral can be performed analytically and expressed as a finite sum of polynomial and trigonometric terms; see Ref. [245] for the full result.

The directional recoil spectrum is then given by

$$
\frac{\mathrm{d}^{2} R}{\mathrm{~d} E_{r} \mathrm{~d} \Omega_{\mathrm{r}}}=\frac{\rho_{0} \sigma_{\mathrm{N}} S(q)}{4 \pi^{3} m_{\chi} \mu_{\mathrm{N}}^{2} v_{\mathrm{esc}}} \sum_{n l m} i^{-l} c_{l n} x_{l n}^{3} j_{l-1}\left(x_{l n}\right) K_{l}^{n}\left[\left(v_{\mathrm{r}}+\mathbf{v}_{\mathrm{lab}} \cdot \hat{\mathbf{r}}\right) / v_{\mathrm{esc}}\right] S_{l m}(\hat{\mathbf{r}}) g_{l m}^{n} .
$$


Although this result may superficially appear somewhat complicated, it is actually surprisingly straightforward. Given a velocity distribution characterized by a finite set of expansion coefficients $g_{l m}^{n}$ (truncated at some maximum $n$ and $l$ ), we have an analytic result for the directional recoil spectrum as a sum of a finite number of terms composed of elementary functions. Such a result allows likelihood-based estimation of the expansion coefficients to be performed efficiently, obviating the need for numerical integration to calculate the predicted directional recoil spectrum during computation of the likelihood.

\section{Discretized decomposition}

As mentioned above, the requirement that the velocity distribution be everywhere positive places a restriction on the possible coefficients $g_{l m}^{n}$. This is a consequence of using the spherical harmonics (which can take negative values) as an angular basis, meaning that any method which makes use of a spherical harmonic decomposition will have a similar requirement. Consequently, for a given set of coefficients $g_{l m}^{n}$, it may be necessary to perform computationally expensive tests to check for unphysical negative values of $f(\mathbf{v})$. Even if such unphysical distributions can be rejected, it is not clear how this problem will affect parameter scans which attempt to reconstruct the coefficients $g_{l m}^{n}$ and therefore the shape of $f(\mathbf{v})$.

An alternative approach [246] is to discretize the velocity distribution in the angular variables, such that $f(\mathbf{v})$ is a function only of $v=|\mathbf{v}|$ within each angular bin. For discretization into $N$ bins, one writes

$$
f(\mathbf{v})=f\left(v, \cos \theta^{\prime}, \phi^{\prime}\right)= \begin{cases}f^{1}(v) & \text { for } \theta^{\prime} \in[0, \pi / N], \\ f^{2}(v) & \text { for } \theta^{\prime} \in[\pi / N, 2 \pi / N], \\ & \vdots \\ f^{k}(v) & \text { for } \theta^{\prime} \in[(k-1) \pi / N, k \pi / N], \\ & \vdots \\ f^{N}(v) & \text { for } \theta^{\prime} \in[(N-1) \pi / N, \pi],\end{cases}
$$

where for simplicity only discretization in the polar angle $\theta^{\prime}$ is considered. The velocity distribution is now described by $N$ functions of $v$, labelled $f^{k}(v)$. Using this decomposition, $f(\mathbf{v})$ is guaranteed to be everywhere positive (and therefore physical) provided that a suitable parametrisation of $f^{k}(v)$ is chosen which is also everywhere positive (for examples, see Refs. [198, 249, 250]).

Because some directional information is discarded in discretizing the velocity distribution, it is expected that the corresponding Radon transform may not provide a close approximation to the true Radon transform. Instead, the integrated Radon transform (IRT) is considered, obtained by integrating $\hat{f}\left(v_{\min }, \hat{\mathbf{r}}\right)$ over the same angular bins as above,

$$
\hat{f}^{j}\left(v_{\min }\right)=\int_{\phi=0}^{2 \pi} \int_{\cos (j \pi / N)}^{\cos ((j-1) \pi / N)} \hat{f}\left(v_{\min }, \hat{\mathbf{r}}\right) \mathrm{d} \cos \theta \mathrm{d} \phi .
$$

Though using the IRT requires that the data be binned in angle, this should reduce the error induced by using the discretized (rather than full) velocity distribution. In the simplest case, $N=1$, the IRT corresponds to the total non-directional rate and induces no discretization error [140]. For $N=2$ (capturing a simple forward-backward asymmetry), the IRTs can be written

$$
\begin{aligned}
& \hat{f}^{1}\left(v_{\min }\right)=4 \pi \int_{v_{\min }}^{\infty} v\left\{\pi f^{1}(v)+\tan ^{-1}\left(\sqrt{v^{2} / v_{\min }^{2}-1}\right)\left[f^{2}(v)-f^{1}(v)\right]\right\} \mathrm{d} v, \\
& \hat{f}^{2}\left(v_{\min }\right)=4 \pi \int_{v_{\min }}^{\infty} v\left\{\pi f^{2}(v)+\tan ^{-1}\left(\sqrt{v^{2} / v_{\min }^{2}-1}\right)\left[f^{1}(v)-f^{2}(v)\right]\right\} \mathrm{d} v .
\end{aligned}
$$

The angular integrals involved in the Radon transform can be performed analytically, leaving only a single integral over $v$ for each IRT. Furthermore, computation of the IRTs can be extended to arbitrary definitions of the angular bins and to arbitrarily high $N$ (see Appendix B of Ref. [246] for explicit expressions).

In order to determine the size of the error induced by the discretization, one can compare the fraction of signal events in each angular bin, calculated using the 'approximate' and 'exact' IRTs. For a given velocity distribution, the 'approximate' IRT is obtained from the discretized velocity distribution as described above, with $f^{k}(v)$ equal 
Dark Matter velocity distribution, including substructures.

The expected reach of directional detection of Dark Matter thus highlights the interest in a worldwide effort toward the construction of large directional detectors.

\section{ACKNOWLEDGEMENTS}

J.B.R.B. acknowledges the support of the Alfred P. Sloan Foundation, and the Research Corporation for Science Advancement. N.B. acknowledges support from the European Research Council through the ERC starting grant WIMPs Kairos. G.B.G. was supported in part by the US Department of Energy under Award Number DE-SC0009937. A.M.G. acknowledges funding from STFC and the Leverhulme Trust. B.J.K. is supported by the European Research Council (ERC) under the EU Seventh Framework Programme (FP7/2007- 2013)/ERC Starting Grant (agreement n. 278234 - 'NewDark' project). B.J.K. is also supported in part by the John Templeton Foundation Grant 48222. J.M. acknowledges support from ERC project no. 279980 S.V. acknowledges support from the U.S. Department of Homeland Security under Award Number 2011-DN-077-ARI050-03 and the U.S. Department of Energy under Award Numbers DE-SC0007852 and DE-SC0010504.

\section{APPENDIX A: TRANSFORMATION FROM THE DETECTOR FRAME TO THE GALACTIC FRAME}

The differential event rate depends on the Radon transform, $\hat{f}$ of the WIMP velocity distribution, which in turn depends on $\hat{\mathbf{r}} \cdot \mathbf{v}_{\text {lab }}$. Hence, in order to compute the differential rate we need to orient the nuclear recoil direction $\hat{\mathbf{r}}$ with respect to $\mathbf{v}_{\text {lab }}$. Here we present complete transformation equations for $\hat{\mathbf{r}}$ and $\mathbf{v}_{\text {lab }}$ to go from the detector frame to the Galactic reference frame. The equations are the same as presented in Appendix A of Ref. [167] except for those describing Earth's revolution.

We define a reference frame fixed to the laboratory and orient its axes so that the $x y$ plane is horizontal, the $x$-axis points North, the $y$-axis points West, and the $z$-axis points to the Zenith. We denote its unit coordinate vectors as $\hat{\mathcal{N}}, \hat{\mathcal{W}}$ and $\hat{\mathcal{Z}}$, respectively. The detector is at some orientation in the laboratory. We define the detector frame with $X, Y, Z$ cartesian axes fixed to the detector. The unit coordinate vectors of the detector frame are $\hat{\mathbf{X}}, \hat{\mathbf{Y}}$ and $\hat{\mathbf{Z}}$. The transformation between the lab frame and the detector frame is given in Eqs. A1 to A3 of Ref. [251] in terms of "direction cosines". $\hat{\mathbf{r}}$ is given in the detector reference frame as $\hat{\mathbf{r}}=r_{X} \hat{\mathbf{X}}+r_{Y} \hat{\mathbf{Y}}+r_{Z} \hat{\mathbf{Z}}$, and we can write it in the lab frame using Eq. A1 of Ref. [251], $\hat{\mathbf{r}}=r_{n} \hat{\mathcal{N}}+r_{w} \hat{\mathcal{W}}+r_{z} \hat{\mathcal{Z}}$, where

$$
\begin{aligned}
r_{n} & =r_{X} \alpha_{X}+r_{Y} \alpha_{Y}+r_{Z} \alpha_{Z}, \\
r_{w} & =r_{X} \beta_{X}+r_{Y} \beta_{Y}+r_{Z} \beta_{Z}, \\
r_{z} & =r_{X} \gamma_{X}+r_{Y} \gamma_{Y}+r_{Z} \gamma_{Z},
\end{aligned}
$$

and $\alpha_{i}, \beta_{i}$ and $\gamma_{i}$ are the direction cosines between the two sets of cartesian coordinates of the lab and detector frames, for $i=X, Y, Z$. For example $\alpha_{Y}$ is the cosine of the angle between the $\hat{\mathcal{N}}$ and $\hat{\mathbf{Y}}$ directions, and $\beta_{Z}$ is the cosine of the angle between the $\hat{\mathcal{W}}$ and $\hat{\mathbf{Z}}$ directions.

We would like to write $\hat{\mathbf{r}}$ in the Galactic reference frame. We proceed by first writing $\hat{\mathbf{r}}$ in the equatorial frame using Eq. A8 of Ref. [251],

$$
\begin{aligned}
\hat{\mathbf{r}} & =\left[-r_{n} \sin \left(\lambda_{\text {lab }}\right) \cos \left(t_{\text {lab }}^{\circ}\right)+r_{w} \sin \left(t_{\text {lab }}^{\circ}\right)+r_{z} \cos \left(\lambda_{\text {lab }}\right) \cos \left(t_{\text {lab }}^{\circ}\right)\right] \hat{\mathbf{x}}_{e} \\
& +\left[-r_{n} \sin \left(\lambda_{\text {lab }}\right) \sin \left(t_{\text {lab }}^{\circ}\right)-r_{w} \cos \left(t_{\text {lab }}^{\circ}\right)+r_{z} \cos \left(\lambda_{\text {lab }}\right) \sin \left(t_{\text {lab }}^{\circ}\right)\right] \hat{\mathbf{y}}_{e} \\
& +\left[r_{n} \cos \left(\lambda_{\text {lab }}\right)+r_{z} \sin \left(\lambda_{\text {lab }}\right)\right] \hat{\mathbf{z}}_{e},
\end{aligned}
$$

where $\hat{\mathbf{x}}_{e}, \hat{\mathbf{y}}_{e}$, and $\hat{\mathbf{z}}_{e}$ are the unit coordinate vectors of the geocentric equatorial inertial (GEI) frame: its origin is at the center of the Earth, its $x_{e}$-axis points in the direction of the vernal equinox, its $y_{e}$-axis points to the point on the celestial equator with right ascension $90^{\circ}$ (so that the cartesian frame is right-handed), and its $z_{e}$-axis points to the north celestial pole. $t_{\text {lab }}^{\circ}$ is the laboratory Local Apparent Sidereal Time in degrees, and $\lambda_{\text {lab }}$ is the latitude of the lab.

The transformation from the Galactic frame to the equatorial frame is given by

$$
\left(\begin{array}{c}
\hat{\mathbf{x}}_{e} \\
\hat{\mathbf{y}}_{e} \\
\hat{\mathbf{z}}_{e}
\end{array}\right)=\mathbf{A}_{\mathrm{G}}\left(\begin{array}{c}
\hat{\mathbf{x}}_{g} \\
\hat{\mathbf{y}}_{g} \\
\hat{\mathbf{z}}_{g}
\end{array}\right)
$$


where

$$
\mathbf{A}_{\mathrm{G}}=\left(\begin{array}{ccc}
a_{x} & b_{x} & c_{x} \\
a_{y} & b_{y} & c_{y} \\
a_{z} & b_{z} & c_{z}
\end{array}\right)
$$

and $\hat{\mathbf{x}}_{g}, \hat{\mathbf{y}}_{g}$, and $\hat{\mathbf{z}}_{g}$ are the unit vectors of the Galactic reference frame. Recall the definition of the Galactic coordinate system: its origin is at the position of the Sun, its $x_{g}$-axis points towards the Galactic center, its $y_{g}$-axis points in the direction of the Galactic rotation, and its $z_{g}$-axis points to the north Galactic pole. Note that these coordinates are related to Galactic longitude $l$ and latitude $b$ by $\left(x_{g}, y_{g}, z_{g}\right)=(\cos b \cos l, \cos b \sin l, \sin b)$. We have $\hat{\mathbf{r}}=r_{x g} \hat{\mathbf{x}}_{g}+$ $r_{y g} \hat{\mathbf{y}}_{g}+r_{z g} \hat{\mathbf{z}}_{g}$, where

$$
\begin{aligned}
r_{x g} & =r_{n}\left(-\left[a_{x} \cos \left(t_{\text {lab }}^{\circ}\right)+a_{y} \sin \left(t_{\text {lab }}^{\circ}\right)\right] \sin \left(\lambda_{\text {lab }}\right)+a_{z} \cos \left(\lambda_{\text {lab }}\right)\right)+r_{w}\left(a_{x} \sin \left(t_{\text {lab }}^{\circ}\right)-a_{y} \cos \left(t_{\text {lab }}^{\circ}\right)\right) \\
& +r_{z}\left(\left[a_{x} \cos \left(t_{\text {lab }}^{\circ}\right)+a_{y} \sin \left(t_{\text {lab }}^{\circ}\right)\right] \cos \left(\lambda_{\text {lab }}\right)+a_{z} \sin \left(\lambda_{\text {lab }}\right)\right), \\
r_{y g} & =r_{n}\left(-\left[b_{x} \cos \left(t_{\text {lab }}^{\circ}\right)+b_{y} \sin \left(t_{\text {lab }}^{\circ}\right)\right] \sin \left(\lambda_{\text {lab }}\right)+b_{z} \cos \left(\lambda_{\text {lab }}\right)\right)+r_{w}\left(b_{x} \sin \left(t_{\text {lab }}^{\circ}\right)-b_{y} \cos \left(t_{\text {lab }}^{\circ}\right)\right) \\
& +r_{z}\left(\left[b_{x} \cos \left(t_{\text {lab }}^{\circ}\right)+b_{y} \sin \left(t_{\text {lab }}^{\circ}\right)\right] \cos \left(\lambda_{\text {lab }}\right)+b_{z} \sin \left(\lambda_{\text {lab }}\right)\right), \\
r_{z g} & =r_{n}\left(-\left[c_{x} \cos \left(t_{\text {lab }}^{\circ}\right)+c_{y} \sin \left(t_{\text {lab }}^{\circ}\right)\right] \sin \left(\lambda_{\text {lab }}\right)+c_{z} \cos \left(\lambda_{\text {lab }}\right)\right)+r_{w}\left(c_{x} \sin \left(t_{\text {lab }}^{\circ}\right)-c_{y} \cos \left(t_{\text {lab }}^{\circ}\right)\right) \\
& +r_{z}\left(\left[c_{x} \cos \left(t_{\text {lab }}^{\circ}\right)+c_{y} \sin \left(t_{\text {lab }}^{\circ}\right)\right] \cos \left(\lambda_{\text {lab }}\right)+c_{z} \sin \left(\lambda_{\text {lab }}\right)\right) .
\end{aligned}
$$

As done in Ref. [252], the transformation matrix $\mathbf{A}_{\mathrm{G}}$ can be found using the definition of the Galactic pole and center in the International Celestial Reference System (ICRS). The north Galactic pole can be defined by right ascension $\alpha_{G}=192^{\circ} .85948$ and declination $\delta_{G}=+27^{\circ} .12825$ in the ICRS. The origin of Galactic longitude is defined by the Galactic longitude of the ascending node of the Galactic plane on the equator of ICRS, which is $l_{\Omega}=32^{\circ} .93192$. The angles $\alpha_{G}, \delta_{G}$ and $l_{\Omega}$ should be regarded as exact quantities, and they can be used to compute the transformation matrix $\mathbf{A}_{\mathrm{G}}[252]$,

$$
\mathbf{A}_{\mathrm{G}}=\left(\begin{array}{ccc}
-0.0548755604 & +0.4941094279 & -0.8676661490 \\
-0.8734370902 & -0.4448296300 & -0.1980763734 \\
-0.4838350155 & +0.7469822445 & +0.4559837762
\end{array}\right) .
$$

The direction of $\hat{\mathbf{r}}$ in the Galactic rest frame can be specified by the Galactic longitude $\left(l_{r}\right)$ and latitude $\left(b_{r}\right)$ of $\hat{\mathbf{r}}$,

$$
r_{x g}=\cos b_{r} \cos l_{r}, \quad r_{y g}=\cos b_{r} \sin l_{r}, \quad r_{z g}=\sin b_{r},
$$

where $r_{x g}, r_{y g}$, and $r_{z g}$ are given in terms of $r_{n}, r_{w}$, and $r_{z}$ in Eq. A5.

The velocity of the lab with respect to the center of the Galaxy can be divided into four components: the Galactic rotation velocity $\mathbf{v}_{c}$ at the position of the Sun (or Local Standard of Rest (LSR) velocity), Sun's peculiar velocity $\mathbf{v}_{s}$ in the LSR, Earth's translational velocity $\mathbf{v}_{\mathrm{e}, \text { rev }}$ with respect to the Sun, and the velocity of Earth's rotation around itself $\mathbf{v}_{\mathrm{e}, \text { rot }}$. In the following sections, we will write each component of $\mathbf{v}_{\text {lab }}$ in the Galactic frame and compute,

$$
\hat{\mathbf{r}} \cdot \mathbf{v}_{\text {lab }}=\hat{\mathbf{r}} \cdot \mathbf{v}_{c}+\hat{\mathbf{r}} \cdot \mathbf{v}_{s}+\hat{\mathbf{r}} \cdot \mathbf{v}_{\mathrm{e}, \text { rev }}+\hat{\mathbf{r}} \cdot \mathbf{v}_{\mathrm{e}, \text { rot }} .
$$

\section{Galactic rotation}

The velocity of the Galactic rotation $\mathbf{v}_{c}$ is defined in the Galactic reference frame,

$$
\mathbf{v}_{c}=v_{c} \hat{\mathbf{y}}_{g},
$$

where $v_{c}$ is the Galactic rotation speed (i.e. the local circular speed), and $\hat{\mathbf{y}}_{g}$ is in the direction of the Galactic rotation. We have,

$$
\hat{\mathbf{r}} \cdot \mathbf{v}_{c}=r_{y g} v_{c},
$$

where $r_{y g}$ is given in Eq. A5. The standard value of $v_{c}$ is $220 \mathrm{~km} \mathrm{~s}^{-1}$ with an order $10 \%$ statistical error $[15,16,97]$. Without assuming a flat rotation curve for the MW, there is a larger spread of values for $v_{c}$ [98]. 
The Sun's peculiar velocity in the LSR is,

$$
\mathbf{v}_{s}=U \hat{\mathbf{x}}_{g}+V \hat{\mathbf{y}}_{g}+W \hat{\mathbf{z}}_{g}
$$

and we have,

$$
\hat{\mathbf{r}} \cdot \mathbf{v}_{s}=r_{x g} U+r_{y g} V+r_{z g} W .
$$

Ref. [253] finds $(U, V, W)_{\odot}=\left(11.1_{-0.75}^{+0.69}, 12.24_{-0.47}^{+0.47}, 7.25_{-0.36}^{+0.37}\right) \mathrm{km} \mathrm{s}^{-1}$, with additional systematic uncertainties $\sim(1,2,0.5) \mathrm{km} \mathrm{s}^{-1}$. These values are extremely insensitive to the metallicity gradient within the disk.

\section{Earth's revolution}

The velocity of the Earth's revolution around the Sun including first order corrections from the eccentricity of the Earth's orbit and the precession of the equinoxes is given by [112] (see also Ref. [113] for equivalent results but without including the effect of the precession),

$$
\begin{aligned}
\mathbf{v}_{\mathrm{e}, \mathrm{rev}} & =v_{\oplus}\left(\cos \beta_{x}\left[\sin \left(L-\lambda_{x}\right)+e \sin \left(L+g-\lambda_{x}\right)\right] \hat{\mathbf{x}}_{g}\right. \\
& +\cos \beta_{y}\left[\sin \left(L-\lambda_{y}\right)-e \sin \left(L+g-\lambda_{y}\right)\right] \hat{\mathbf{y}}_{g} \\
& \left.+\cos \beta_{z}\left[\sin \left(L-\lambda_{z}\right)-e \sin \left(L+g-\lambda_{z}\right)\right] \hat{\mathbf{z}}_{g}\right),
\end{aligned}
$$

where $v_{\oplus}=29.79 \mathrm{~km} \mathrm{~s}^{-1}$ is the orbital speed of the Earth, $e=0.0167023$ is the ellipticity of the Earth's orbit [254], and $\left(\beta_{i}, \lambda_{i}\right)$ are the ecliptic latitudes and longitudes of the $\left(\hat{\mathbf{x}}_{g}, \hat{\mathbf{y}}_{g}, \hat{\mathbf{z}}_{g}\right)$ axes (given below). The mean longitude of the Sun, $L$, and the mean anomaly of the Earth in it's orbit around the Sun, $g$ are given by [254],

$$
\begin{aligned}
L & =279^{\circ} .344+0.9856474 d, \\
g & =356^{\circ} .154+0.9856003 d .
\end{aligned}
$$

Here $d$ is the fractional day number from December 31st 2014 at 0 UT (Universal Time in hours). For example, on January 1 st 2015 the fractional day number is $d=1$ at $0 \mathrm{UT}$, and $d=1.5$ at 12:00 UT. For a given calendar date with year $Y$, month $M$, day of month $D$, and time of the day UT (in hours), the fractional day number is given by [255]

$$
d=\lfloor 365.25 \tilde{Y}\rfloor+\lfloor 30.61(\tilde{M}+1)\rfloor+D+\frac{\mathrm{UT}}{24}-736041
$$

where the floor function $\lfloor x\rfloor$ is the largest integer not greater than $x$. For $M=1$ or $2, \tilde{Y}=Y-1$ and $\tilde{M}=M+12$. For $M>2, \tilde{Y}=Y$ and $\tilde{M}=M$. For example on 7 th of March $2015(Y=2015, M=3, D=7)$ at $10: 00 \mathrm{UT}$, we have $d=66.42$.

The ecliptic latitudes and longitudes of the $\left(\hat{\mathbf{x}}_{g}, \hat{\mathbf{y}}_{g}, \hat{\mathbf{z}}_{g}\right)$ axes are

$$
\begin{aligned}
& \left(\beta_{x}, \lambda_{x}\right)=\left(5^{\circ} .538,267^{\circ} .050\right)+\left(0^{\circ} .013,1^{\circ} .397\right) T \\
& \left(\beta_{y}, \lambda_{y}\right)=\left(-59^{\circ} .574,347^{\circ} .546\right)+\left(0^{\circ} .002,1^{\circ} .375\right) T, \\
& \left(\beta_{z}, \lambda_{z}\right)=\left(29^{\circ} .811,180^{\circ} .234\right)+\left(0^{\circ} .001,1^{\circ} .404\right) T,
\end{aligned}
$$

where $T=d / 36525$ is the epoch of date and accounts for the precession of the equinoxes. Ref. [112] finds that the consequences of ignoring the $T$ dependence of $\beta_{i}$ for the amplitude and peak time of the annual modulation are very small. The $T$ dependence becomes important over a timescale of decades from the reference time of December 31 st 2014 at $0 \mathrm{UT}$ in which $d$ is calculated.

We can compute $\hat{\mathbf{r}} \cdot \mathbf{v}_{\mathrm{e}, \mathrm{rev}}$ as

$$
\begin{aligned}
\hat{\mathbf{r}} \cdot \mathbf{v}_{\mathrm{e}, \mathrm{rev}} & =v_{\oplus}\left(\cos \beta_{x}\left[\sin \left(L-\lambda_{x}\right)+e \sin \left(L+g-\lambda_{x}\right)\right] r_{x g}\right. \\
& +\cos \beta_{y}\left[\sin \left(L-\lambda_{y}\right)-e \sin \left(L+g-\lambda_{y}\right)\right] r_{y g} \\
& \left.+\cos \beta_{z}\left[\sin \left(L-\lambda_{z}\right)-e \sin \left(L+g-\lambda_{z}\right)\right] r_{z g}\right) .
\end{aligned}
$$




\section{Earth's rotation}

The velocity of Earth's rotation around its axis is given by

$$
\mathbf{v}_{\mathrm{e}, \mathrm{rot}}=-v_{\mathrm{e}, \mathrm{rot}}^{\mathrm{eq}} \cos \lambda_{\mathrm{lab}} \hat{\mathcal{W}}
$$

where $v_{\mathrm{e}, \text { rot }}^{\mathrm{eq}}$ is the Earth's rotation speed at the equator, and is defined as $v_{\mathrm{e}, \text { rot }}^{\mathrm{eq}}=2 \pi R_{\oplus} /(1$ sidereal day). The Earth's equatorial radius is $R_{\oplus}=6378.137 \mathrm{~km}$, and one sidereal day is $23.9344696 \mathrm{hr}=86164 \mathrm{~s}$. Therefore $v_{\mathrm{e}, \text { rot }}^{\text {eq }}=0.465102$ $\mathrm{km} \mathrm{s}^{-1}$.

We use Eq. A8 of Ref. [251] to write $\hat{\mathcal{W}}$ in terms of the equatorial frame coordinates,

$$
\mathbf{v}_{\mathrm{e}, \mathrm{rot}}=-v_{\mathrm{e}, \mathrm{rot}}^{\mathrm{eq}} \cos \lambda_{\mathrm{lab}}\left(\sin \left(t_{\text {lab }}^{\circ}\right) \hat{\mathbf{x}}_{e}-\cos \left(t_{\text {lab }}^{\circ}\right) \hat{\mathbf{y}}_{e}\right)
$$

Using the the Galactic to equatorial transformation, we have

$$
\begin{aligned}
\mathbf{v}_{\mathrm{e}, \mathrm{rot}} & =-v_{\mathrm{e}, \mathrm{rot}}^{\mathrm{eq}} \cos \lambda_{\text {lab }}\left\{\left(a_{x} \sin \left(t_{\text {lab }}^{\circ}\right)-a_{y} \cos \left(t_{\text {lab }}^{\circ}\right)\right) \hat{\mathbf{x}}_{g}\right. \\
& \left.+\left(b_{x} \sin \left(t_{\text {lab }}^{\circ}\right)-b_{y} \cos \left(t_{\text {lab }}^{\circ}\right)\right) \hat{\mathbf{y}}_{g}+\left(c_{x} \sin \left(t_{\text {lab }}^{\circ}\right)-c_{y} \cos \left(t_{\text {lab }}^{\circ}\right)\right) \hat{\mathbf{z}}_{g}\right\} .
\end{aligned}
$$

Thus we can compute $\hat{\mathbf{r}} \cdot \mathbf{v}_{\mathrm{e}, \text { rot }}$ as

$$
\begin{aligned}
\hat{\mathbf{r}} \cdot \mathbf{v}_{\mathrm{e}, \text { rot }} & =-v_{\mathrm{e}, \text { rot }}^{\mathrm{eq}} \cos \lambda_{\text {lab }}\left\{\left(a_{x} \sin \left(t_{\text {lab }}^{\circ}\right)-a_{y} \cos \left(t_{\text {lab }}^{\circ}\right)\right) r_{x g}\right. \\
& \left.+\left(b_{x} \sin \left(t_{\text {lab }}^{\circ}\right)-b_{y} \cos \left(t_{\text {lab }}^{\circ}\right)\right) r_{y g}+\left(c_{x} \sin \left(t_{\text {lab }}^{\circ}\right)-c_{y} \cos \left(t_{\text {lab }}^{\circ}\right)\right) r_{z g}\right\} .
\end{aligned}
$$

Inserting $r_{x g}, r_{y g}$ and $r_{z g}$ from Eq. A5, we find

$$
\begin{aligned}
\hat{\mathbf{r}} \cdot \mathbf{v}_{\mathrm{e}, \mathrm{rot}} & =-v_{\mathrm{e}, \mathrm{rot}}^{\mathrm{eq}} \cos \lambda_{\mathrm{lab}}\left\{\left(\left[a_{y}^{2}+b_{y}^{2}+c_{y}^{2}-a_{x}^{2}-b_{x}^{2}-c_{x}^{2}\right] \cos \left(t_{\mathrm{lab}}^{\circ}\right) \sin \left(t_{\mathrm{lab}}^{\circ}\right) \sin \lambda_{\mathrm{lab}}\right.\right. \\
& +\left[a_{x} a_{y}+b_{x} b_{y}+c_{x} c_{y}\right]\left(\cos ^{2}\left(t_{\mathrm{lab}}^{\circ}\right)-\sin ^{2}\left(t_{\mathrm{lab}}^{\circ}\right)\right) \sin \lambda_{\mathrm{lab}} \\
& \left.+\left[a_{x} a_{z}+b_{x} b_{z}+c_{x} c_{z}\right] \sin \left(t_{\mathrm{lab}}^{\circ}\right) \cos \lambda_{\mathrm{lab}}+\left[a_{y} a_{z}+b_{y} b_{z}+c_{y} c_{z}\right] \cos \left(t_{\mathrm{lab}}^{\circ}\right) \cos \lambda_{\mathrm{lab}}\right) r_{n} \\
& +\left(\left[a_{x}^{2}+b_{x}^{2}+c_{x}^{2}\right] \sin ^{2}\left(t_{\mathrm{lab}}^{\circ}\right)+\left[a_{y}^{2}+b_{y}^{2}+c_{y}^{2}\right] \cos ^{2}\left(t_{\mathrm{lab}}^{\circ}\right)\right. \\
& \left.-2\left[a_{x} a_{y}+b_{x} b_{y}+c_{x} c_{y}\right] \cos \left(t_{\mathrm{lab}}^{\circ}\right) \sin \left(t_{\mathrm{lab}}^{\circ}\right)\right) r_{w} \\
& +\left(\left[a_{x}^{2}+b_{x}^{2}+c_{x}^{2}-a_{y}^{2}-b_{y}^{2}-c_{y}^{2}\right] \cos \left(t_{\mathrm{lab}}^{\circ}\right) \sin \left(t_{\mathrm{lab}}^{\circ}\right) \cos \lambda_{\mathrm{lab}}\right. \\
& +\left[a_{x} a_{y}+b_{x} b_{y}+c_{x} c_{y}\right]\left(\sin ^{2}\left(t_{\mathrm{lab}}^{\circ}\right)-\cos ^{2}\left(t_{\mathrm{lab}}^{\circ}\right)\right) \cos \lambda_{\mathrm{lab}} \\
& \left.\left.+\left[a_{x} a_{z}+b_{x} b_{z}+c_{x} c_{z}\right] \sin \left(t_{\mathrm{lab}}^{\circ}\right) \sin \lambda_{\mathrm{lab}}-\left[a_{y} a_{z}+b_{y} b_{z}+c_{y} c_{z}\right] \cos \left(t_{\mathrm{lab}}^{\circ}\right) \sin \lambda_{\mathrm{lab}}\right) r_{z}\right\} .
\end{aligned}
$$

From Eq. A6, we have $a_{i} a_{j}+b_{i} b_{j}+c_{i} c_{j}=\delta_{i j}$ where $i, j=x, y, z$. Therefore, Eq. A25 will be simplified to

$$
\hat{\mathbf{r}} \cdot \mathbf{v}_{\mathrm{e}, \text { rot }}=-r_{w} v_{\mathrm{e}, \mathrm{rot}}^{\mathrm{eq}} \cos \lambda_{\mathrm{lab}}
$$

\section{Total Velocity}

Using Eqs. A10, A12, A20 and A26 we can compute $\hat{\mathbf{r}} \cdot \mathbf{v}_{\text {lab }}$ as

$$
\begin{aligned}
\hat{\mathbf{r}} \cdot \mathbf{v}_{\text {lab }} & =r_{x g}\left(U+v_{\oplus} \cos \beta_{x}\left[\sin \left(L-\lambda_{x}\right)+e \sin \left(L+g-\lambda_{x}\right)\right]\right) \\
& +r_{y g}\left(v_{c}+V+v_{\oplus} \cos \beta_{y}\left[\sin \left(L-\lambda_{y}\right)+e \sin \left(L+g-\lambda_{y}\right)\right]\right) \\
& +r_{z g}\left(W+v_{\oplus} \cos \beta_{z}\left[\sin \left(L-\lambda_{z}\right)+e \sin \left(L+g-\lambda_{z}\right)\right]\right)-r_{w} v_{\mathrm{e}, \text { rot }}^{\text {eq }} \cos \lambda_{\text {lab }},
\end{aligned}
$$


where $r_{x g}, r_{y g}$, and $r_{z g}$ can be written in terms of $r_{n}, r_{w}$, and $r_{z}$ using Eq. A5. We can also write $\hat{\mathbf{r}} \cdot \mathbf{v}_{\text {lab }}$ in terms of only $r_{x g}, r_{y g}$, and $r_{z g}$ using Eqs. A10, A12, A20 and A24,

$$
\begin{aligned}
\hat{\mathbf{r}} \cdot \mathbf{v}_{\text {lab }} & =r_{x g}\left(U+v_{\oplus} \cos \beta_{x}\left[\sin \left(L-\lambda_{x}\right)+e \sin \left(L+g-\lambda_{x}\right)\right]\right. \\
& \left.-v_{\mathrm{e}, \text { rot }}^{\mathrm{eq}} \cos \lambda_{\mathrm{lab}}\left(a_{x} \sin \left(t_{\text {lab }}^{\circ}\right)-a_{y} \cos \left(t_{\text {lab }}^{\circ}\right)\right)\right) \\
& +r_{y g}\left(v_{c}+V+v_{\oplus} \cos \beta_{y}\left[\sin \left(L-\lambda_{y}\right)+e \sin \left(L+g-\lambda_{y}\right)\right]\right. \\
& \left.-v_{\mathrm{e}, \text { rot }}^{\mathrm{eq}} \cos \lambda_{\mathrm{lab}}\left(b_{x} \sin \left(t_{\text {lab }}^{\circ}\right)-b_{y} \cos \left(t_{\text {lab }}^{\circ}\right)\right)\right) \\
& +r_{z g}\left(W+v_{\oplus} \cos \beta_{z}\left[\sin \left(L-\lambda_{z}\right)+e \sin \left(L+g-\lambda_{z}\right)\right]\right. \\
& \left.-v_{\mathrm{e}, \text { rot }}^{\mathrm{eq}} \cos \lambda_{\text {lab }}\left(c_{x} \sin \left(t_{\text {lab }}^{\circ}\right)-c_{y} \cos \left(t_{\text {lab }}^{\circ}\right)\right)\right)
\end{aligned}
$$

[1] P. A. R. Ade et al., arXiv:1502.01589

[2] R. Mandelbaum, U. Seljak, G. Kauffmann, C. M. Hirata and J. Brinkmann, Mon. Not. Roy. Astron. Soc. 368 (2006) 715

[3] M. Boylan-Kolchin et al., Astrophys.J. 768 (2013)140

[4] G. Bertone, D. Hooper and J. Silk, Phys. Rept. 405 (2005) 279

[5] G. Jungman, M. Kamionkowski and K. Griest, Phys. Rept. 267 (1996) 195

[6] G. Steigman and M. S. Turner, Nucl. Phys. B 253 (1985) 375.

[7] E. Halkiadakis, G. Redlinger and D. Shih, Ann. Rev. Nucl. Part. Sci. 64 (2014) 319

[8] T. A. Porter, R. P. Johnson and P. W. Graham, Ann. Rev. Astron. Astrophys. 49 (2011) 155

[9] L. Baudis, Phys. Dark Univ. 1 (2012) 94

[10] J. Billard, L. Strigari and E. Figueroa-Feliciano, Phys. Rev. D 89 (2014) 023524

[11] K. Freese, J. A. Frieman and A. Gould, Phys. Rev. D 37 (1988) 3388.

[12] K. Freese, M. Lisanti and C. Savage, Rev. Mod. Phys. 85 (2013) 1561

[13] A. K. Drukier, K. Freese and D. N. Spergel, Phys. Rev. D 33 (1986) 3495.

[14] J. D. Lewin and P. F. Smith, Astropart. Phys. 6 (1996) 87.

[15] J. Bovy et al., Astrophys. J. 759 (2012) 131

[16] J. Bovy, D. W. Hogg and H. -W. Rix, Astrophys. J. 704 (2009) 1704

[17] D. N. Spergel, Phys. Rev. D 37 (1988) 1353.

[18] T. Naka and K. Miuchi (Eds), Proc. of CYGNUS 2013, fourth International Conference on Directional Detection of Dark Matter (Toyama, Japan, June 2013), J. Phys. Conf. Series 469 (2013)

[19] S. Ahlen, et al., Int. J. Mod. Phys. A 25 (2010) 1

[20] F. Mayet and D. Santos (Eds), Proc. of CYGNUS 2011, third International Conference on Directional Detection of Dark Matter (Aussois, France, June 2011), EAS Publications Series 53 (2012)

[21] J. B. R. Battat et al., JINST 9 (2014) 11, P11004

[22] S. Ahlen et al., Phys. Lett. B 695 (2011) 124

[23] J. P. Lopez et al., Nucl. Instrum. Meth. A 696 (2012) 121

[24] E. Daw et al., Astropart. Phys. 35 (2012) 397

[25] S. E. Vahsen et al., EAS Publ. Ser. 53 (2012) 43

[26] D. Santos et al., EAS Publ. Ser. 53 (2012) 25

[27] K. Miuchi et al., Phys. Lett. B 686 (2010) 11

[28] J. B. R. Battat et al., Nucl. Instrum. Meth. A 755 (2014) 6.

[29] J. B. R. Battat et al., Phys. Dark Univ. 9-10 (2015) 1

[30] N. D'Ambrosio et al., JINST 9 (2014) 01, C01043.

[31] T. Naka et al., Nucl. Instrum. Meth. A 718 (2013) 519.

[32] D. Nygren, J. Phys. Conf. Series 460 (2013) 012006

[33] V. M. Gehman, A. Goldschmidt, D. Nygren, C. A. B. Oliveira and J. Renner, JINST 8 (2013) C10001.

[34] G. Mohlabeng, K. Kong, J. Li, A. Para and J. Yoo, JHEP 1507 (2015) 092

[35] G. Cavoto, E. N. M. Cirillo, F. Cocina, J. Ferretti and A. D. Polosa, arXiv:1602.03216

[36] F. Capella et al. Eur. Phys. J. C. 73 (2013) 2276

[37] H. Sekiya, talk at Cygnus 2015, 5th International Conference on Directional Detection of Dark Matter (Los Angeles, CA, USA, June 2015)

[38] A. K. Drukier et al., Int. J. Mod. Phys. A 29 (2014) 1443007

[39] S. Y. Wang, arXiv:1509.08801 [physics.ins-det].

[40] C. T. Chiang, M. Kamionkowski and G. Z. Krnjaic, Phys. Dark Univ. 1 (2012) 109

[41] Q. Riffard et al., arXiv:1504.05865 
[42] T. Asada, talk at Cygnus 2015, 5th International Conference on Directional Detection of Dark Matter (Los Angeles, CA, USA, June 2015)

[43] J. Billard, Phys. Rev. D 91 (2015) 2, 023513

[44] J. Billard, F. Mayet and D. Santos, JCAP 1204 (2012) 006

[45] P. Majewski et al., Astropart. Phys. 34 (2010) 284

[46] D. Dujmic et al., Nucl. Instrum. Meth. A 584 (2008) 327

[47] S. Burgos et al., Astropart. Phys. 31 (2010) 261

[48] A. M. Green and B. Morgan, Phys. Rev. D 77 (2008) 027303

[49] J. Billard, PhD thesis, University of Grenoble, June 2012

[50] L. G. Christophorou and J. K. Olthoff, Fundamental Electron Interactions with Plasma Processing Gases, Springer, 2004

[51] P. M. Lewis, S. E. Vahsen, I. S. Seong, M. T. Hedges, I. Jaegle and T. N. Thorpe, Nucl. Instrum. Meth. A 789, 81 (2015)

[52] D. P. Snowden-Ifft, Rev. Sci. Instrum. 85, 013303 (2014).

[53] C. J. Martoff et al., Nucl. Instrum. Methods Phys. Res. A 440 (2000) 355

[54] T. Ohnuki, C. J. Martoff and D. Snowden-Ifft, Nucl. Instrum. Methods Phys. Res. A 463 (2001) 142

[55] C. J. Martoff et al., Nucl. Instrum. Methods Phys. Res. A 598 (2009) 501

[56] M. P. Dion et al., Nucl. Instrum. Methods Phys. Res. A 648 (2011) 186

[57] D. P. Snowden-Ifft and J.-L Gauvreau, Rev. Sci. Instrum. 84 (2013) 053304

[58] J. R. Ellis \& R. A. Flores, Phys. Lett. B 263 (1991) 259

[59] N. S. Phan, R. J. Lauer, E. R. Lee, D. Loomba, J. A. J. Matthews and E. H. Miller, arXiv:1510.02170 [physics.ins-det].

[60] D. Loomba, N. S. Phan, E. Miller, talk at Cygnus 2015, 5th International Conference on Directional Detection of Dark Matter (Los Angeles, CA, USA, June 2015)

[61] C. Golabek et al., Nucl. Instrum. Meth. A 678 (2012) 33

[62] J. Brack et al., JINST 9 (2014) P07021

[63] J. B. R. Battat et al., JINST 9 (2014) P11004

[64] J. B. R. Battat et al., Nucl. Instr. Meth. Phys. Res. A 794 (2015) 3346

[65] J. Brack et al., Phys. Procedia 61 (2015) 130137

[66] O. Guillaudin et al., EAS Publications Series 53 (2012) 119-127

[67] J. Billard, F. Mayet and D. Santos, JCAP 1207 (2012) 020

[68] J. P. Lopez et al., Phys. Procedia 37 (2012) 575.

[69] D. Mei and A. Hime, Phys. Rev. D 73 (2006) 053004

[70] B. Morgan, A. M. Green and N. J. C. Spooner, Phys. Rev. D 71 (2005) 103507

[71] J. Billard, F. Mayet, J. F. Macias-Perez and D. Santos, Phys. Lett. B 691 (2010) 156

[72] A. Gutlein et al., Astropart. Phys. 34 (2010) 90

[73] J. Monroe and P. Fisher, Phys. Rev. D 76 (2007) 033007

[74] F. Ruppin, J. Billard, E. Figueroa-Feliciano and L. Strigari, Phys. Rev. D 90 (2014) 083510

[75] D. P. Snowden-Ifft, C. J. Martoff and J. M. Burwell, Phys. Rev. D 61 (2000) 101301

[76] D. P. Snowden-Ifft, arXiv:1308.0354 [physics.ins-det].

[77] N. J. C. Spooner, talk at CYGNUS 2013, fourth International Conference on Directional Detection of Dark Matter (Toyama, Japan, June 2013), http://ppwww.phys.sci.kobe-u.ac.jp/ newage/cygnus2013/

[78] F. Mayet, O. Guillaudin, C. Grignon, C. Koumeir, D. Santos, P. Colas and I. Giomataris, J. Phys. Conf. Ser. 179 (2009) 012011

[79] D. Santos et al., J. Phys. Conf. Ser. 309 (2011) 012014

[80] F. J. Iguaz et al., JINST 6 (2011) P07002

[81] E. Ferrer-Ribas et al., EAS Publ. Ser. 53 (2012) 165

[82] J. P. Richer et al., Nucl. Instrum. Meth. A 620 (2010) 470

[83] O. Bourrion et al., Nucl. Instrum. Meth. A 662 (2010) 207

[84] F. Mayet et al., J. Phys. Conf. Ser. 469 (2013) 012006

[85] J. Billard et al., JINST 9 (2014) 01, P01013

[86] D. Santos et al., J. Phys. Conf. Ser. 469 (2013) 012002

[87] K. Nakamura et al., PTEP 2015 (2015) 4, 043 F01.

[88] D. Dujmic et al., Astropart. Phys. 30 (2008) 58

[89] D. Dujmic et al., Nucl. Instrum. Meth. A 584 (2008) 327

[90] C. Deaconu, G. Druitt, R. Eggleston, P. Fisher, J. Lopez, J. Monroe, H. Tomita and E. Zayas, Phys. Procedia 61 (2015) 39.

[91] C. S. Deaconu, PhD thesis, MIT, 2015

[92] A. C. Kaboth, PhD thesis, MIT, 2012

[93] M. Leyton, Proceedings of the 15th International Conference on Topics in Astroparticle and Underground Physics (TAUP 2015), in press

[94] S. E. Vahsen et al., Nucl. Instrum. Meth. A 788 (2015) 95

[95] Readout technologies for directional WIMP dark matter detection, in preparation

[96] Fifth International Conference on Directional Detection of Dark Matter (Occidental College, Los Angeles, CA, USA, June 2015), http://www.cygnus2015.com/

[97] F. J. Kerr and D. Lynden-Bell, Mon. Not. Roy. Astron. Soc. 221 (1986) 1023.

[98] P. J. McMillan and J. J. Binney, Mon. Not. Roy. Astron. Soc. 402, 934 (2010) 
[99] J. Binney and S. Tremaine, Galactic dynamics, Princeton University Press (2008)

[100] T. Piffl et al., Astron. Astrophys. 562 (2014) A91

[101] P. J. McMillan, Mon. Not. Roy. Astron. Soc. 414 (2011) 2446

[102] R. Catena and P. Ullio, JCAP 1008 (2010) 004

[103] J. F. Navarro, C. S. Frenk and S. D. M. White, Astrophys. J. 462 (1996) 563

[104] J. Bovy and H. W. Rix, Astrophys. J. 779 (2013) 115

[105] F. Nesti and P. Salucci, JCAP 1307 (2013) 016

[106] M. Pato, F. Iocco and G. Bertone, JCAP 1512 (2015) 12, 001

[107] M. Weber and W. de Boer, Astron. Astrophys. 509 (2010) A25

[108] P. Salucci, F. Nesti, G. Gentile and C. F. Martins, Astron. Astrophys. 523 (2010) A83

[109] S. Garbari, C. Liu, J. I. Read and G. Lake, Mon. Not. Roy. Astron. Soc. 425 (2012) 1445

[110] M. S. Alenazi and P. Gondolo, Phys. Rev. D 74 (2006) 083518

[111] K. Griest, Phys. Rev. D 37 (1988) 2703.

[112] C. McCabe, JCAP 1402, 027 (2014)

[113] S. K. Lee, M. Lisanti, B. R. Safdi, JCAP 1311, 033 (2013)

[114] M. Kuhlen, M. Vogelsberger and R. Angulo, Phys. Dark Univ. 1 (2012) 50

[115] M. Vogelsberger et al., Mon. Not. Roy. Astron. Soc. 395 (2009) 797

[116] M. Kuhlen et al., JCAP 1002 (2010) 030

[117] Y. - Y. Mao, L. E. Strigari and R. H. Wechsler, Phys. Rev. D 89 (2014) 063513

[118] M. Lisanti, L. E. Strigari, J. G. Wacker and R. H. Wechsler, Phys. Rev. D 83 (2011) 023519

[119] F. S. Ling, E. Nezri, E. Athanassoula and R. Teyssier, JCAP 1002 (2010) 012

[120] M. Kuhlen, A. Pillepich, J. Guedes and P. Madau, Astrophys. J. 784 (2014) 161

[121] I. Butsky et al., arXiv:1503.04814

[122] N. Bozorgnia et al., arXiv:1601.04707

[123] C. Kelso, C. Savage, M. Valluri, K. Freese, G. S. Stinson and J. Bailin, arXiv:1601.04725

[124] J. D. Sloane, M. R. Buckley, A. M. Brooks and F. Governato, arXiv:1601.05402

[125] B. Moore et al., Phys. Rev. D 64 (2001) 063508

[126] M. Vogelsberger et al., Monthly Notices of the Royal Astronomical Society 395 (2009) 797-811

[127] F. S. Ling, E. Nezri, E. Athanassoula and R. Teyssier, JCAP 1002 (2010) 012

[128] M. Kuhlen, M. Lisanti and D. N. Spergel, Phys. Rev. D 86 (2012) 063505

[129] M. Lisanti and D. N. Spergel, Phys. Dark Univ. 1 (2012) 155

[130] C. W. Purcell, A. R. Zentner and M. -Y. Wang, JCAP 1208 (2012) 027

[131] K. Freese, P. Gondolo and H. J. Newberg, Phys. Rev. D 71 (2005) 043516

[132] J. I. Read et al., Mon. Not. Roy. Astron. Soc. 389 (2008) 1041

[133] J. I. Read et al., Mon. Not. Roy. Astron. Soc. 397 (2009) 44

[134] C. W. Purcell, J. S. Bullock and M. Kaplinghat, Astrophys. J. 703 (2009) 2275

[135] G. R. Ruchti et al., Mon. Not. Roy. Astron. Soc. 450 (2015) 3, 2874

[136] J. I. Read, J. Phys. G 41 (2014) 063101

[137] A. M. Green, Mod. Phys. Lett. A 27 (2012) 1230004

[138] A. H. G. Peter, V. Gluscevic, A. M. Green, B. J. Kavanagh and S. K. Lee, Phys. Dark Univ. 5-6 (2014) 45

[139] M. W. Goodman and E. Witten, Phys. Rev. D 31 (1985) 3059.

[140] P. Gondolo, Phys. Rev. D 66 (2002) 103513

[141] J. Engel, S. Pittel and P. Vogel, Int. J. Mod. Phys. E 1 (1992) 1.

[142] Q. Riffard, F. Mayet, G. Bélanger, M.-H. Genest and D. Santos, arXiv:1602.01030

[143] R. Helm, Phys. Rev. 104 (1956) 1466

[144] G. Co', V. De Donno, M. Anguiano and A. M. Lallena, JCAP 1211 (2012) 010

[145] D. G. Cerdeno, M. Fornasa, J.-H. Huh and M. Peiro, Phys. Rev. D 87 (2013) 2, 023512

[146] V. A. Bednyakov and F. Simkovic, Phys. Part. Nucl. 36 (2005) 131

[147] E. Moulin, F. Mayet and D. Santos, Phys. Lett. B 614 (2005) 143

[148] C. Cheung, L. J. Hall, D. Pinner and J. T. Ruderman, JHEP 1305 (2013) 100

[149] H. Baer, A. Belyaev, T. Krupovnickas and J. O'Farrill, JCAP 0408 (2004) 005

[150] F. Mayet et al., Phys. Lett. B 538 (2002) 257

[151] G. Bertone, D. G. Cerdeno, M. Fornasa, L. Pieri, R. R. de Austri and R. Trotta, Phys. Rev. D 85 (2012) 055014

[152] A. Bottino, F. Donato, N. Fornengo and S. Scopel, Phys. Rev. D 77 (2008) 015002

[153] A. Arbey, M. Battaglia and F. Mahmoudi, Phys. Rev. D 89 (2014) 7, 077701

[154] L. Bergstrom, T. Bringmann and J. Edsjo, Phys. Rev. D 83 (2011) 045024

[155] D. Albornoz Vasquez, G. Belanger, J. Billard and F. Mayet, Phys. Rev. D 85 (2012) 055023

[156] J. Beringer et al., Phys. Rev. D 86 (2012) 010001

[157] J. Billard, F. Mayet and D. Santos, Phys. Rev. D 83 (2011) 075002

[158] S. K. Lee and A. H. G. Peter, JCAP 1204 (2012) 029

[159] J. Fan, M. Reece and L. T. Wang, JCAP 1011, 042 (2010)

[160] J. B. Dent, L. M. Krauss, J. L. Newstead and S. Sabharwal, arXiv:1505.03117 [hep-ph].

[161] N. Anand, A. L. Fitzpatrick and W. C. Haxton, Phys. Rev. C 89 (2014) 6, 065501

[162] A. L. Fitzpatrick, W. Haxton, E. Katz, N. Lubbers and Y. Xu, JCAP 1302 (2013) 004, arXiv:1211.2818 
[163] R. Catena and B. Schwabe, JCAP 1504 (2015) 042

[164] K. Schneck et al. [SuperCDMS Collaboration], Phys. Rev. D 91 (2015) 9, 092004

[165] B. J. Kavanagh, Phys. Rev. D 92 (2015) 2, 023513

[166] R. Catena, JCAP 1507 (2015) 07, 026

[167] N. Bozorgnia, G. B. Gelmini and P. Gondolo, JCAP 1206 (2012) 037

[168] N. Bozorgnia, G. B. Gelmini and P. Gondolo, JCAP 1208 (2012) 011

[169] P. Sikivie and S. Wick, Phys. Rev. D 66, 023504 (2002)

[170] S. K. Lee, M. Lisanti, A. H. G. Peter and B. R. Safdi, Phys. Rev. Lett. 112, 011301 (2014)

[171] N. Bozorgnia and T. Schwetz JCAP 1408 (2014) 013

[172] E. Del Nobile, G. B. Gelmini and S. J. Witte JCAP 1508 (2015) 08, 041

[173] S. Yellin, Phys. Rev. D 66, 032005 (2002)

[174] S. Henderson, J. Monroe and P. Fisher, Phys. Rev. D 78 (2008) 015020

[175] D. Y. Akimov et al., Phys. Lett. B 692 (2010) 180

[176] J. Billard, F. Mayet and D. Santos, Phys. Rev. D 82 (2010) 055011

[177] P. Grothaus, M. Fairbairn and J. Monroe, Phys. Rev. D 90 (2014) 5, 055018

[178] M. S. Alenazi and P. Gondolo, Phys. Rev. D 77 (2008) 043532

[179] C. J. Copi, J. Heo and L. M. Krauss, Phys. Lett. B 461 (1999) 43

[180] C. J. Copi and L. M. Krauss, Phys. Rev. D 63 (2001) 043507

[181] A. M. Green and B. Morgan, Phys. Rev. D 81 (2010) 061301

[182] N. I. Fisher, T. Lewis and B. J. J. Embleton, Statistical analysis of spherical data, CUP (1987).

[183] J. Billard, Q. Riffard, F. Mayet and D. Santos, Phys. Lett. B 718 (2013) 1171

[184] A. M. Green and B. Morgan, Astropart. Phys. 27 (2007) 142

[185] B. Morgan and A. M. Green, Phys. Rev. D 72 (2005) 123501

[186] Lord Rayleigh, Phil. Mag. 10, 73 (1880); Nature 72 (1905) 318; Phil. Mag. 37 (1918) 321

[187] K. V. Mardia and P. Jupp, Directional Statistics, Wiley, Chichester (2002).

[188] J. Billard, F. Mayet and D. Santos, Phys. Rev. D 85 (2012) 035006

[189] C. A. J. O'Hare, A. M. Green, J. Billard, E. Figueroa-Feliciano and L. E. Strigari, Phys. Rev. D 92 (2015) 6 , 063518

[190] J. H. Davis, JCAP 1503 (2015) 012

[191] A.L. Read, "Modified Frequentist Analysis of Search Results (The CLS Method)", Workshop on Confidence Limits, CERN (2000).

[192] N. W. Evans, C. M. Carollo and P. T. de Zeeuw, Mon. Not. Roy. Astron. Soc. 318 (2000) 1131

[193] N. Bernal, A. Goudelis, Y. Mambrini and C. Munoz, JCAP 0901 (2009) 046

[194] M. Drees and C. L. Shan, JCAP 0806 (2008) 012

[195] C. L. Shan, Mod. Phys. Lett. A 25 (2010) 951

[196] M. Drees and C. L. Shan, JCAP 0706 (2007) 011

[197] A. M. Green, JCAP 0807 (2008) 005

[198] B. J. Kavanagh and A. M. Green, Phys. Rev. Lett. 111 (2013) 031302

[199] B. J. Kavanagh, Phys. Rev. D 89 (2014) 085026

[200] B. J. Kavanagh, M. Fornasa and A. M. Green, Phys. Rev. D 91 (2015) 103533

[201] C. Arina, G. Bertone and H. Silverwood, Phys. Rev. D 88 (2013) 013002

[202] G. Bertone et al., Phys. Rev. D 82 (2010) 055008

[203] L. E. Strigari and R. Trotta, JCAP 0911 (2009) 019

[204] Y. T. Chou and C. L. Shan, JCAP 1008 (2010) 014

[205] C. L. Shan, JCAP 1006 (2010) 029

[206] C. L. Shan, New J. Phys. 11 (2009) 105013

[207] P. Salucci, F. Nesti, G. Gentile and C. F. Martins, Astron. Astrophys. 523 (2010) A83

[208] L. Bergstrom, T. Bringmann and J. Edsjo, Phys. Rev. D 83 (2011) 045024

[209] H. Baer, E. -K. Park and X. Tata, New J. Phys. 11 (2009) 105024

[210] R. Laha, Phys. Rev. D 92 (2015) 083509

[211] D. Tucker-Smith and N. Weiner, Phys. Rev. D 64, 043502 (2001)

[212] C. Savage, G. Gelmini, P. Gondolo and K. Freese, JCAP 904 (2009) 10

[213] E. Aprile et al. [XENON100 Collaboration], Phys. Rev. D84 (2011) 061101

[214] D. P. Finkbeiner, T. Lin and N. Weiner, Phys. Rev. D 80, 115008 (2009)

[215] M. Lisanti and J. G. Wacker, Phys. Rev. D 81 (2010) 096005

[216] R. Agnese et al. [CDMS Collaboration], Phys. Rev. Lett. 111 (2013) 251301

[217] C. E. Aalseth et al. [CoGeNT Collaboration], Phys. Rev. Lett. 106 (2011) 131301

[218] G. Angloher et al., Eur. Phys. J. C 72 (2012) 1971

[219] C. E. Aalseth et al. Phys. Rev. Lett. 107 (2011) 141301

[220] R. Bernabei et al. [DAMA and LIBRA Collaborations], Eur. Phys. J. C 67 (2010) 39

[221] C. Arina, Phys. Rev. D 86 (2012) 123527

[222] D. S. Akerib et al. [LUX Collaboration], Phys.Rev.Lett. 112 (2014) 091303

[223] G. Angloher et al. [CRESST-II Collaboration], Eur. Phys. J. C 74 (2014) 3184

[224] J. H. Davis, C. McCabe and C. Boehm, JCAP 1408 (2014) 014

[225] D. Albornoz Vásquez, G. Belanger, C. Boehm, A. Pukhov and J. Silk, Phys. Rev. D 82 (2010) 115027 
[226] A. Bottino, N. Fornengo and S. Scopel, Phys. Rev. D 67 (2003) 063519

[227] J. F. Gunion, D. Hooper and B. McElrath, Phys. Rev. D 73 (2006) 015011

[228] D. Das and U. Ellwanger, JHEP 1009 (2010) 085

[229] K. M. Zurek, Phys. Rept. 537 (2014) 91

[230] B. Morgan and A. M. Green, Phys. Rev. D 86 (2012) 083544

[231] G. S. Watson, Geophys. Suppl. Mon. Not. Roy. Soc. 7, 160 (1956)

[232] K. V. Mardia and P. Jupp, Directional Statistics, Wiley, Chichester (2002)

[233] J. Tatarowicz, C.J. Martoff, Astropart. Phys. 35 (2011) 235

[234] I. Jaegle et al., EAS Publ. Ser. 53 (2012) 111

[235] N. S. Phan talk at Astroparticle 2014, A joint TevPA/IDM Conference (Amsterdam, Netherlands, June 2014)

[236] C. J. Martoff et al., Nucl. Instrum. Meth. A 440 (2000) 355

[237] C. K. Shalem et al., Nucl. Instrum. Meth. A 558 (2006) 468

[238] A. M. Green, JCAP 1010 (2010) 034

[239] C. Savage, K. Freese and P. Gondolo, Phys. Rev. D 74 (2006) 043531

[240] C. A. J. O'Hare and A. M. Green, Phys. Rev. D 90 (2014) 123511

[241] E. Aprile et al. [XENON100 Collaboration], Astropart. Phys. 35 (2012) 573

[242] M. Maciejewski et al., Mon. Not. Roy. Astron. Soc. 415 (2011) 2475

[243] G. Cowan, K. Cranmer, E. Gross and O. Vitells, Eur. Phys. J. C 71 (2011) 1554

[244] D. S. M. Alves, S. E. Hedri and J. G. Wacker, arXiv:1204.5487 [astro-ph.GA].

[245] S. K. Lee, JCAP 1403 (2014) 047

[246] B. J. Kavanagh, JCAP 1507 (2015) 07, 019

[247] R. W. Michie, MNRAS 125 (1963) 125

[248] M. Kuhlen, J. Diemand, P. Madau and M. Zemp, J. Phys. Conf. Ser. 125 (2008) 012008

[249] B. J. Kavanagh and A. M. Green, Phys. Rev. D 86 (2012) 065027

[250] A. H. G. Peter, Phys. Rev. D 83 (2011) 125029

[251] N. Bozorgnia, G. B. Gelmini and P. Gondolo, Phys. Rev. D 84 (2011) 023516

[252] The Hipparcos and Tycho Catalogues, Volume 1, Section 1.5, Transformation of Astrometric Data and Associated Error Propagation, ESA Publications Division, c/o ESTEC, Noordwijk, The Netherlands, 1997, http://www.rssd.esa.int/SAgeneral/Projects/Hipparcos/ CATALOGUE_VOL1/sect1_05.pdf.

[253] R. Schoenrich, J. Binney and W. Dehnen, Mon. Not. Roy. Astron. Soc. 403 (2010) 1829

[254] The astronomical almanac, U.K. Hydrographic Office, 2015.

[255] J. Meeus Astronomical algorithms, Willmann-Bell, Inc., 2000, Second English edition.

[256] Section IIIF presents a non-relativistic effective framework that enables studies beyond standard SI and SD interactions.

[257] As discussed in Sec. ??, for very low recoil energies the rate is maximum in a ring around the average WIMP arrival direction $[167,251]$. 